\title{
HEMISPHERIC CENTER FOR ENVIRONMENTAL TECHNOLOGY
}

\section{MONTHLY PROGRESS REPORT}

FISCAL YEAR 1999

\section{DE-FG21-95EW55094}
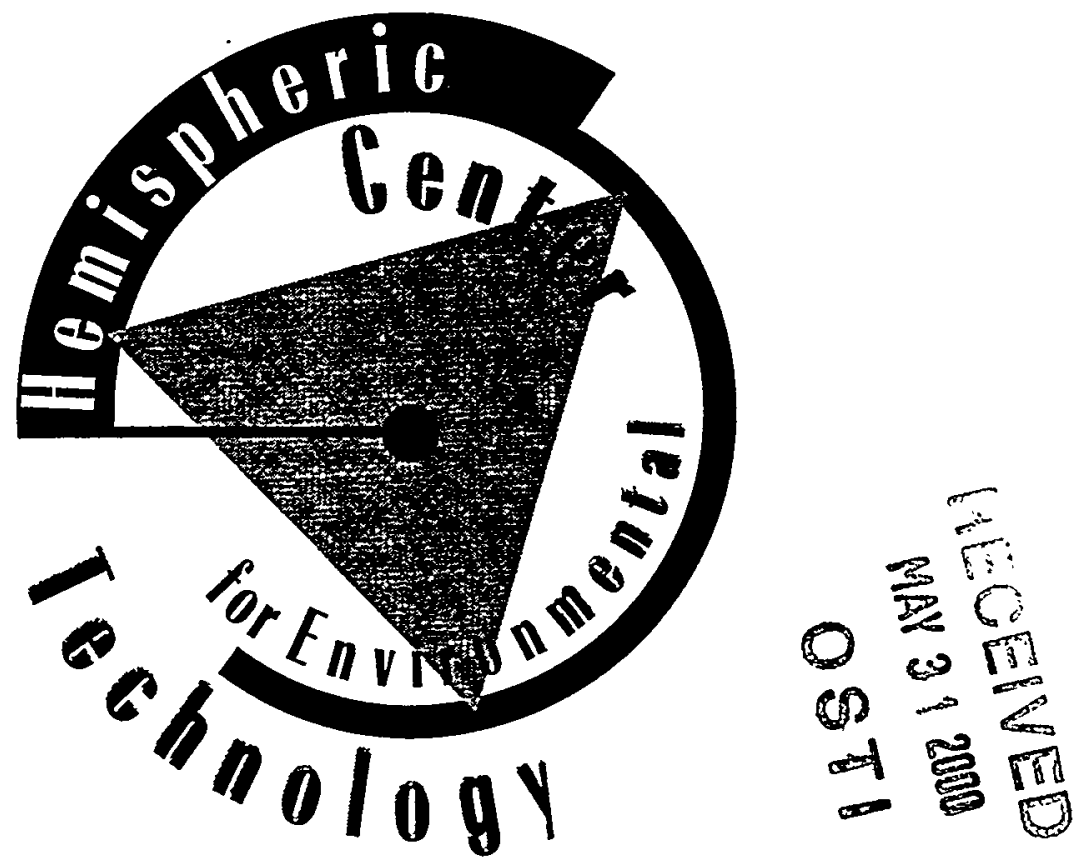

\section{JANUARY 1999}

FIU Principal Investigator

Focus Area Technical Lead Program Officers 


\section{DISCLAIMER}

This report was prepared as an account of work sponsored by an agency of the United States Government. Neither the United States Government nor any agency thereof, nor any of their employees, make any warranty, express or implied, or assumes any legal liability or responsibility for the accuracy, completeness, or usefulness of any information, apparatus, product, or process disclosed, or represents that its use would not infringe privately owned rights. Reference herein to any specific commercial product, process, or service by trade name, trademark, manufacturer, or otherwise does not necessarily constitute or imply its endorsement, recommendation, or favoring by the United States Government or any agency thereof. The views and opinions of authors expressed herein do not necessarily state or reflect those of the United States Government or any agency thereof. 


\section{DISCLAIMER}

Portions of this document may be illegible in electronic image products. Images are produced from the best available original document. 


\title{
HEMISPHERIC CENTER FOR ENVIRONMENTAL TECHNOLOGY MONTHLY PROGRESS REPORT
}

\author{
FISCAL YEAR 1999
}

DE. F HO1-95EU55094
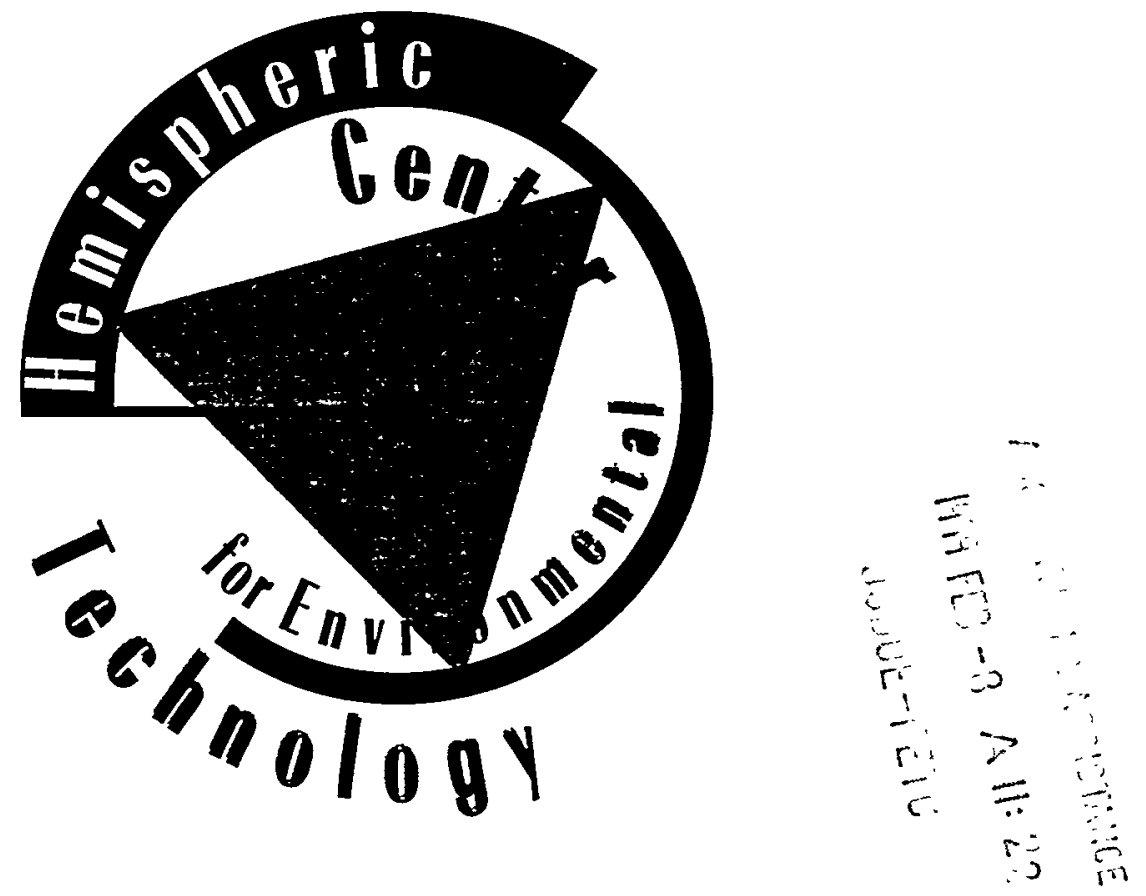

\section{JANUARY 1999}

FIU Principal Investigator

Focus Area Technical Lead Program Officers
M.A. Ebadian

Paul Hart

John Wengle

Karl-Heinz Frohne 


\section{SUMMARY}

- FIU-HCET participated in an ICT meeting at Mound during the second week of December and presented a brief videotape of the testing of the Robotic Climber technology. During this meeting, FIU-HCET proposed the TechXtract technology for possible testing at Mound and agreed to develop a five-page proposal for review by team members.

- FIU-HCET provided assistance to Bartlett Inc. and General Lasertronics Corporation in developing a proposal for a Program Opportunity Notice (PON). The proposal was submitted by these companies on January 5, 1999.

- The search for new equipment dismantlement technologies is continuing. The following vendors have responded to requests for demonstration:

- LUMONICS, Laser Solutions technology

- CRYO-BEAM, Cryogenic cutting technology

- Waterjet Technology Association, Waterjet Cutting technology

- DIAJET, Waterjet Cutting technology.

- Based on the tasks done in FY98, FIU-HCET is working closely with Numatec Hanford Corporation (NHC) and Pacific Northwest National Laboratory (PNNL) to revise the plan and scope of work of the pipeline plugging project in FY99, which involves activities of lab-scale flow loop experiments and a large-scale demonstration test bed. 


\section{TABLE OF CONTENTS}

1. DEACTIVATION AND DECOMMISSIONING (D\&D) FOCUS AREA ............................5 5

Deactivation and Decommissioning Technology Assessment Program ...................... 6

Integrated Vertical and Overhead Decontamination System .................................. 17

Large-Scale Demonstration and Deployment Project-

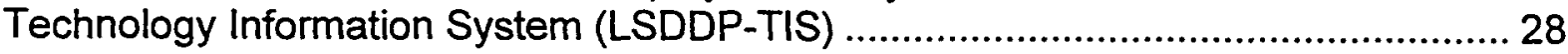

Ex-Situ Large-Bore Pipe Decontamination and Characterization System ................. 31

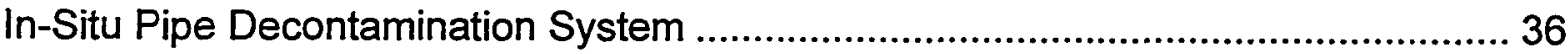

Deactivation and Decommissioning Technology Opportunities for

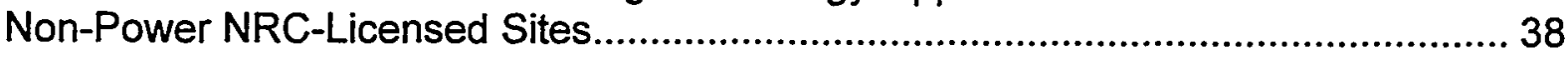

Life-Cycle Cost Analysis for Radioactively Contaminated Scrap Metal .................... 49

Legacy Waste Disposition for the Oak Ridge Reservation ................................... 52

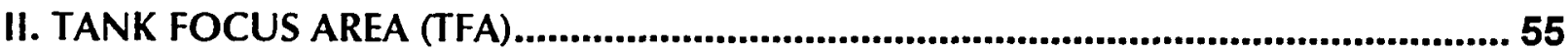

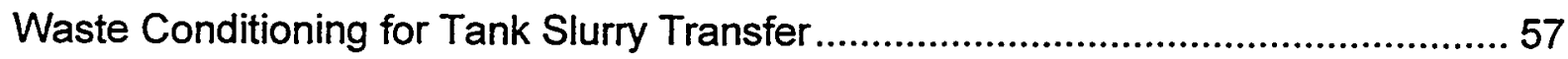

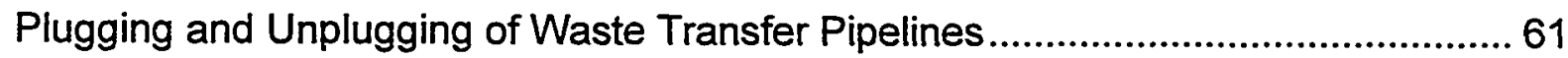

Investigation of Waste Glass Pouring Process Over a Knife Edge ........................... 69

III. CHARACTERIZATION, MONITORING, AND SENSOR TECHNOLOGY ................... 77

Online Measurement of the Progress of Decontamination ...................................... 79

Remote Surveillance of Facilities Awaiting Deactivation and Decommissioning ...... 81

Measurement of Alpha Contamination on Contaminated

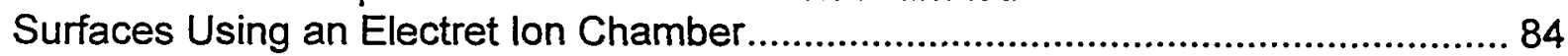

Review of Current Characterization and Monitoring Practices at DOE Sites ............. 87

Demonstration and Deployment of CMST-CP Technologies.................................. 89

Identification of DOE's Post-Closure Monitoring Needs and Requirements .............. 91

IV. INTERNATIONAL TECHNOLOGY INTEGRATION (ITI).............................................93

Opportunities to Market U.S. Technologies Throughout the Western Hemisphere ... 94 
FIU Principal Investigator FIU D\&D Program Manager Focus Area Technical Lead Program Officers
M.A. Ebadian

Rob Rose

Paul W. Hart

John Wengle

Karl-Heinz Frohne

http://www.hcet.fiu.edu 


\section{Deactivation and Decommissioning \\ Technology Assessment Program}

Project Number: HCET-1995-D038

\section{Project objectives}

The Deactivation and Decommissioning (D\&D) Technology Assessment Program (TAP) was developed to provide detailed, comparable data for environmental technologies and to disseminate this data to D\&D professionals in a manner that will facilitate the review and selection of technologies to perform decontamination and decommissioning. The objectives for this project include the following:

- Determine technology needs through review of the Site Technology Coordination Group (STCG) information and other applicable web sites and needs databases.

- Perform a detailed review of industries that perform similar activities as those required in D\&D operations to identify additional technologies.

- Define the technology assessment program for characterization and waste management problem sets.

- Define the data management program for characterization, dismantlement, and waste management problem sets.

- Evaluate baseline and innovative technologies under standard test conditions at Florida International University's Hemispheric Center for Environmental Technology (FIU-HCET) and other locations and to collect data in the areas of performance, cost, health and safety, operations and maintenance, and primary and secondary waste generation.

- Continue to locate, verify, and incorporate technology performance data from other sources into the multimedia information system.

- Develop the conceptual design for a dismantlement technology decision analysis tool for dismantlement technologies.

\section{Major milestones}

\begin{tabular}{|c|c|c|c|}
\hline Milestone No. & Milestone Description & Completion Criteria & Status \\
\hline D038-MI & $\begin{array}{l}\text { Publication of the Technology } \\
\text { Assessment Reports }\end{array}$ & $\begin{array}{l}\text { Completion of } 16 \\
\text { technology } \\
\text { demonstrations and each } \\
\text { summarized in monthly } \\
\text { reports within } 60 \text { days } \\
\text { after the demonstration. }\end{array}$ & $\begin{array}{l}\text { Two technologies } \\
\text { evaluated for various } \\
\text { applications for a total } \\
\text { of three demonstrations. }\end{array}$ \\
\hline$\overline{\mathrm{D} 038-\mathrm{M} 2}$ & $\begin{array}{l}\text { Test Plan for Characterization } \\
\text { Technologies Assessment } \\
\text { Program }\end{array}$ & $\begin{array}{l}\text { Characterization } \\
\text { Technology Test Plan } \\
\text { Approved }\end{array}$ & $\begin{array}{l}\text { Under development. } \\
\text { Scheduled completion } \\
4 / 30 / 99 \text {. }\end{array}$ \\
\hline$\overline{\mathrm{D} 038-\mathrm{M} 3}$ & $\begin{array}{l}\text { Test Plan for Waste } \\
\text { Management Technologies } \\
\text { Assessment Program }\end{array}$ & $\begin{array}{l}\text { Waste Management } \\
\text { Technology Test Plan } \\
\text { Approved }\end{array}$ & $\begin{array}{l}\text { Scheduled start 3/2/99. } \\
\text { Scheduled completion } \\
6 / 30 / 99 \text {. }\end{array}$ \\
\hline
\end{tabular}




\begin{tabular}{|c|l|l|l|}
\hline Milestone No. & \multicolumn{1}{|c|}{ Milestone Description } & Completion Criteria & \multicolumn{1}{c|}{ Status } \\
\hline D038-M4 & $\begin{array}{l}\text { Access to the multimedia } \\
\text { information system web-based } \\
\text { user interface for } \\
\text { dismantlement }\end{array}$ & $\begin{array}{l}\text { Assignment of user } \\
\text { name and passwords to } \\
\text { DDFA provided } \\
\text { distribution list: }\end{array}$ & $\begin{array}{l}\text { Design started 12/7/98. } \\
\text { Scheduled completion } \\
10 / 1 / 99 .\end{array}$ \\
\hline D038-M5 & $\begin{array}{l}\text { Access to the information } \\
\text { system characterization } \\
\text { database }\end{array}$ & $\begin{array}{l}\text { Assignment of user } \\
\text { name and passwords to } \\
\text { DDFA provided } \\
\text { distribution list. }\end{array}$ & $\begin{array}{l}\text { Scheduled start 7/6/99. } \\
\text { Scheduled completion } \\
10 / 29 / 99 .\end{array}$ \\
\hline D038-M6 & $\begin{array}{l}\text { Report on the information } \\
\text { system waste management } \\
\text { design }\end{array}$ & $\begin{array}{l}\text { Final copy of report sent } \\
\text { to DDFA. }\end{array}$ & $\begin{array}{l}\text { Scheduled start 10/1/99. } \\
\text { Scheduled completion } \\
10 / 29 / 99 .\end{array}$ \\
\hline D038-M7 & $\begin{array}{l}\text { Report on the decision analysis } \\
\text { tool for dismantlement design }\end{array}$ & $\begin{array}{l}\text { Final copy of report sent } \\
\text { to DDFA. }\end{array}$ & $\begin{array}{l}\text { Scheduled start 7/6/99. } \\
\text { Scheduled completion } \\
10 / 29 / 99 .\end{array}$ \\
\hline
\end{tabular}

\section{Significant events}

- Mississippi State University - DIAL's draft report on the demonstration of their FTP technology for survey of walls during scabbling was reviewed and commented on by FIU-HCET. The final report is due in early February. Figures 1 and 2 illustrate the DIAL-FTP technology during the wall survey demonstration at FIU-HCET in December 1998. FIU-HCET's summary of the FTP demonstration is found in Appendix A.

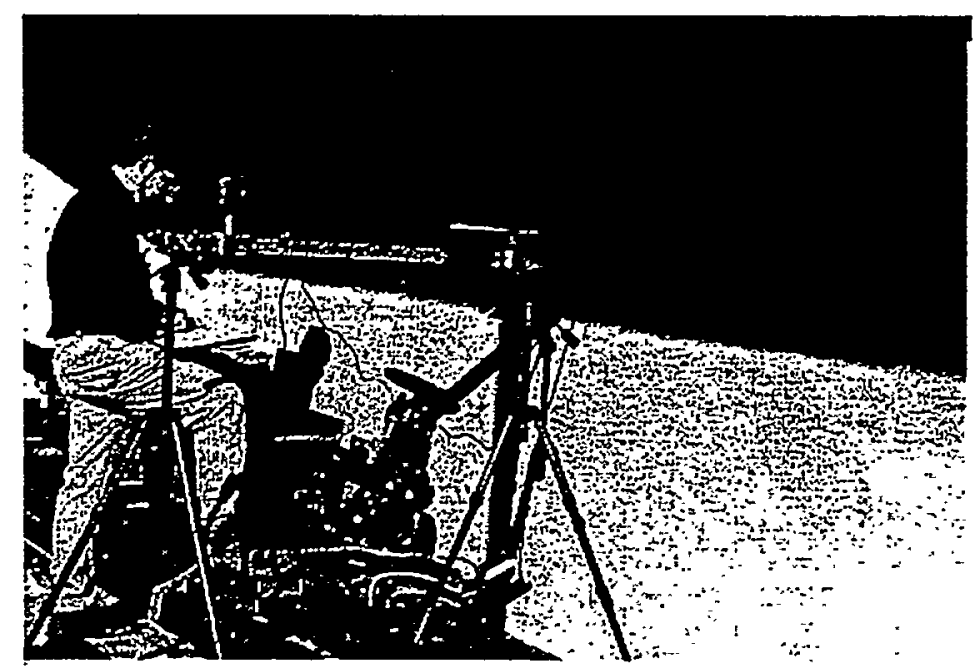

Figure 1. Mississippi State University-DIAL's FTP Technology System (Projector, Camera, and Laptop Computer). 


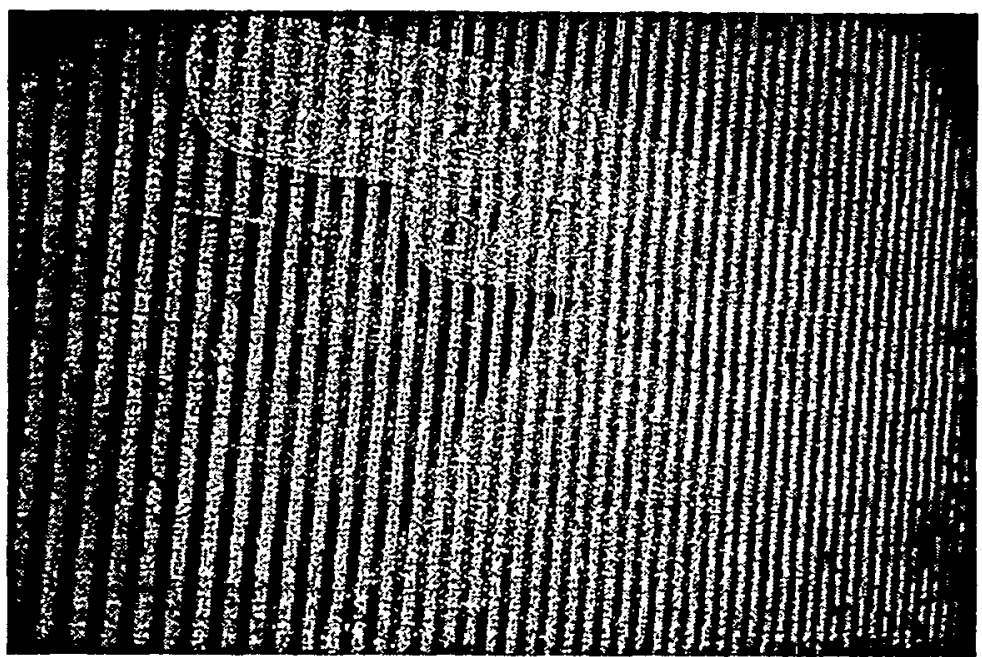

Figure 2. Light patterns projected by FTP system on surface surveyed.

- The statement of work for the diamond wire cutting demonstration at Pacific Northwest National Laboratory (PPPL) was prepared by FIU-HCET, reviewed by PPPL test engineers and AEA Technology, and has been sent to FIU procurement. Six vendors have been identified and will be sent the Request for Proposal.

- Preliminary report on the demonstration of the Robotic Climber ${ }^{\mathrm{TM}}$ (see Figure 3) on four steel plates, one concrete floor, one concrete wall, one brick wall, and one uncoated concrete floor has been completed. The vendor, Bartlett Nuclear Inc., was given 30 days for review. The attachment provided in the hard copy of this monthly report and electronically linked to this document (Attachment-Robotic climber H-1 data entry form mod.doc) contains the data obtained from these demonstrations.

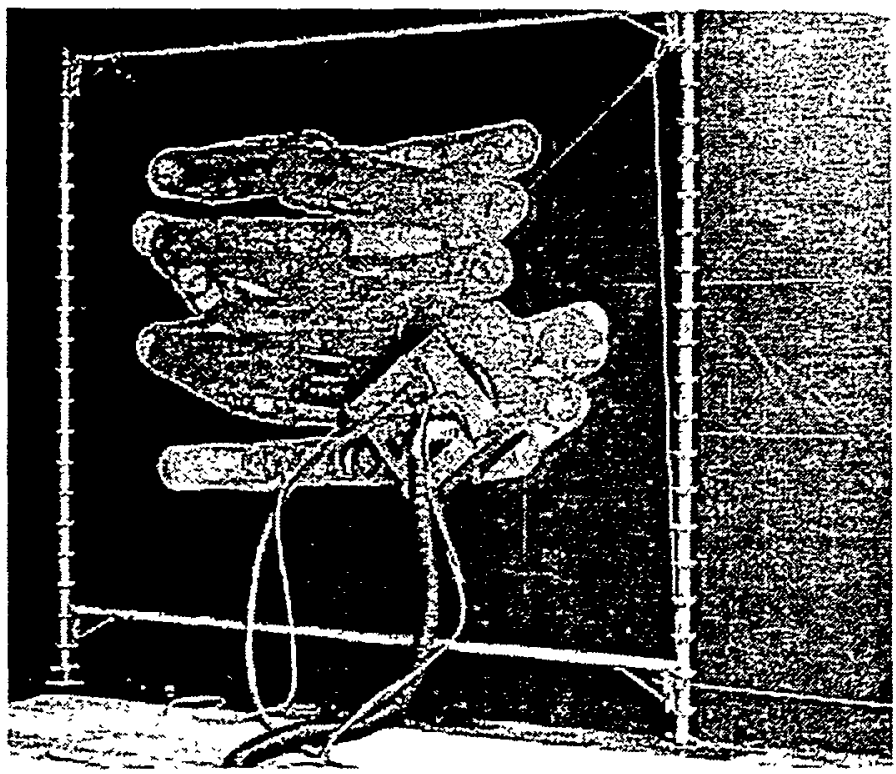

Figure 3. Bartlett Robotic Climber on coated concrete wall. 
- The search for new equipment dismantlement (i.e., pipes, I-beams) technologies is continuing. The following vendors have responded to requests for demonstration:

\begin{tabular}{|l|l|l|}
\hline Vendor & Technology & Status \\
\hline LUMONICS & Laser Solutions & Sent an invitation to bid. \\
\hline CRYO-BEAM & Cryogenic Cutting & $\begin{array}{l}\text { Will send technical information package } \\
\text { to FIU-HCET. }\end{array}$ \\
\hline $\begin{array}{l}\text { Waterjet Technology } \\
\text { Association }\end{array}$ & Waterjet Cutting & $\begin{array}{l}\text { Will send a list of portable waterjet } \\
\text { companies listed under its database. }\end{array}$ \\
\hline DIAJET & Waterjet Cutting & $\begin{array}{l}\text { Will send technical information package } \\
\text { to FIU-HCET. }\end{array}$ \\
\hline
\end{tabular}

- Search for decontamination technologies to be assessed at FIU-HCET is continuing. Potential vendors were contacted for more information. The following vendors were sent Scope of Work and Invitations for Proposals:

\begin{tabular}{|l|l|l|}
\hline Vendor & Technology & Brief description \\
\hline $\begin{array}{l}\text { Practical Machine } \\
\text { Engineering }\end{array}$ & $\begin{array}{l}\text { KIESS Blast } \\
\text { Recovery System } \\
\text { can be used on both vertical surfaces and } \\
\text { horizontal surfaces. It is similar to } \\
\text { Pentek's WallWalker but is not a } \\
\text { scabbling technology. Made in Germany. } \\
\text { The equipment has been used in a } \\
\text { nuclear environment in Germany. }\end{array}$ \\
\hline $\begin{array}{l}\text { ElectroStrip } \\
\text { Corporation }\end{array}$ & ElectroStrip & $\begin{array}{l}\text { TM } \\
\text { Removal of paint by applying cathodic } \\
\text { Preparation } \\
\text { current to painted metal substrate. }\end{array}$ \\
\hline $\begin{array}{l}\text { Universal Iceblast, } \\
\text { Inc. }\end{array}$ & $\begin{array}{l}\text { Searification with a } \\
\text { Solid Steel Drum } \\
\text { Chemical } \\
\text { Treatment }\end{array}$ & $\begin{array}{l}\text { Steel drums with carbide tips, attached to } \\
\text { a fine planer or scarifer. It is capable of } \\
\text { removing paint and concrete. }\end{array}$ \\
\hline Dawson-MacDonald & Multi-Stripper & $\begin{array}{l}\text { The paint is softened with a chemical } \\
\text { treatment and then blasted away using } \\
\text { wet ice. }\end{array}$ \\
\hline & $\begin{array}{l}\text { A hydraulically powered grinding/cutting } \\
\text { machine with dust collector. Able to } \\
\text { remove epoxy paint at a rate of 350-650 } \\
\mathrm{ft}^{2} / \text { hour. }\end{array}$ \\
\hline
\end{tabular}

- Work is ongoing to transfer non-contaminated gloveboxes, raschig ring and annular tanks from Rocky Flats to FIU-HCET to demonstrate dismantlement technologies. The test plan and the setup for these demonstrations are being prepared. Potential technologies are being identified and will be contacted for demonstration. 


\section{Accomplishments and technical progress}

- Under this grant project, which began in FY96, and earlier technology assessment projects funded from other sources, FIU-HCET assessed over 60 innovative/improved and baseline technologies for decontamination and equipment dismantlement under standard, non-nuclear testing conditions. Many of the technologies identified for demonstration at FIU-HCET are selected to address the needs identified in the EM-50 Needs Management System (http://EMNeeds.em.doe.gov/Home/). As a result of these assessments, directly comparable performance data related to operations and maintenance, primary and secondary waste generation, and health and safety have been compiled. This data has been valuable in assessing if a technology meets the screening criteria for those LSDDPs where these technologies are being considered, as well as assisting EM-40 project managers in making decisions on the deployment of innovative technologies. Technology assessment data are managed using a Microsoft Windows-based multimedia information system.

- To date, two technologies have been demonstrated in multiple applications for a total of three assessments in FY99.

\section{Assessment of current status}

This project is on schedule. Two technologies have completed assessment, and the results of these assessments are attached. The test plan for the PPPL diamond wire demonstration is completed, and the Statement of Work is being sent to six potential vendors.

\section{Plans for the next two months}

Activities for the next two months include

- Continuing technology search for FY99 demos. Demonstrating two technologies by the end of March 1999.

- Comparing final results from DIAL FTP with professional surveyors' results. Finish writing FIU-HCET report based on comparative results.

- Drafting test plans for FIU-HCET assessments of facility dismantlement, glovebox dismantlement, and waste management technologies.

- Completing the design of the multimedia information system for dismantlement and beginning programming.

\section{FIU-HCET collaborator}

Susan C. Madaris, (305) 348-3727 
Appendix A. FTP Demonstration Summary

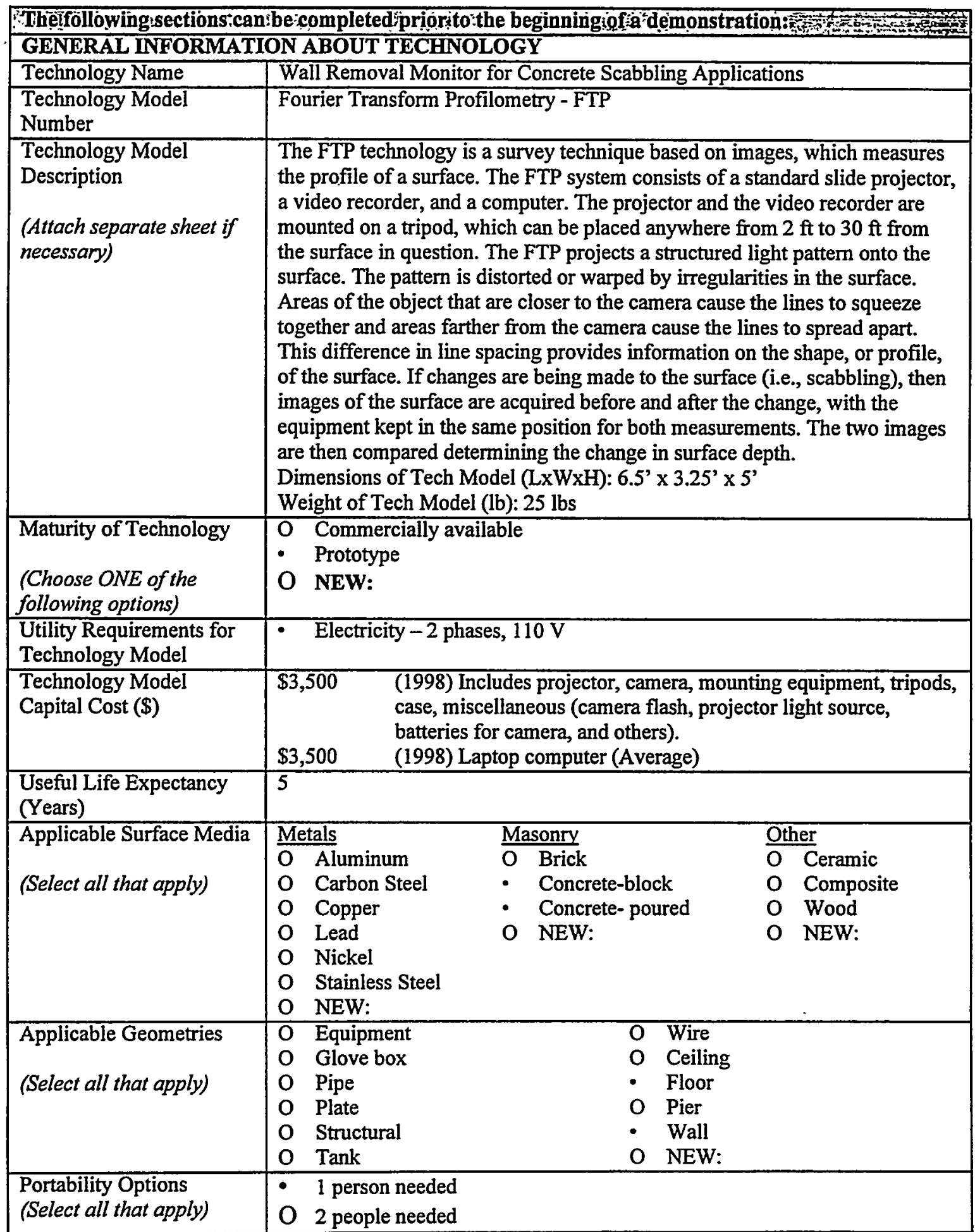


Appendix A. FTP Demonstration Summary (continued)

\begin{tabular}{|c|c|c|}
\hline \multicolumn{3}{|c|}{ MANUFACTURER INFORMATION } \\
\hline Name and Address & $\begin{array}{l}\text { Diagnostic Instrumentation } \\
205 \text { Research Boulevard } \\
\text { Mississippi Research and Te } \\
\text { Starkville, MS } 39759\end{array}$ & $\begin{array}{l}\text { Laboratory - DIAL } \\
\text { Park }\end{array}$ \\
\hline Phone Number(s) & (1) $(601) 325-7376$ & (2) (601) $325-7378$ \\
\hline Fax Number & \multicolumn{2}{|l|}{$(601) 325-8465$} \\
\hline Web-site & \multicolumn{2}{|c|}{ http://www.msstate.edu/dept/dial/ } \\
\hline E-Mail & \multicolumn{2}{|l|}{ Dial@dial.msstate.edu } \\
\hline $\begin{array}{l}\text { Services Available } \\
\text { (Select all that apply) }\end{array}$ & \multicolumn{2}{|c|}{ 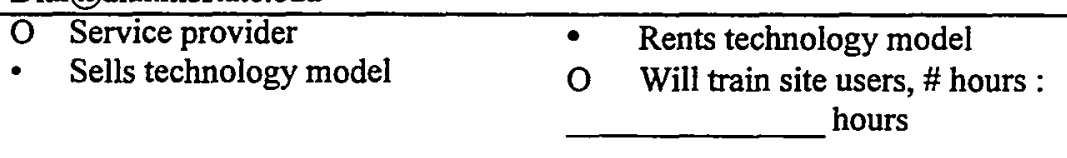 } \\
\hline References & \multicolumn{2}{|c|}{$\begin{array}{l}\text { Henderson, Mark E., December 1997, "Fourier Transform Profilometry with } \\
\text { Code in Matlab," Masters Thesis, Department of Electrical and Computer } \\
\text { Engineering, Mississippi State University. }\end{array}$} \\
\hline Publications & \multicolumn{2}{|l|}{ NA } \\
\hline Photographs/Video & \multicolumn{2}{|c|}{$\begin{array}{l}\text { Check each of the following that was included in package to FIU-HCET: } \\
\text { O Photographs, } \\
\text { O Video, }\end{array}$} \\
\hline \multicolumn{3}{|c|}{ VENDOR INFORMATION } \\
\hline Name and Address & \multicolumn{2}{|c|}{$\begin{array}{l}\text { Diagnostic Instrumentation \& Analysis Laboratory - DIAL } \\
205 \text { Research Boulevard } \\
\text { Mississippi Research and Technology Park } \\
\text { Starkville, MS } 39759\end{array}$} \\
\hline Phone Number(s) & $(1)(601) 325-7376$ & (2) $(601) 325-7378$ \\
\hline Fax Number & \multicolumn{2}{|l|}{$(601) 325-8465$} \\
\hline Web-site & \multicolumn{2}{|c|}{ http://www.msstate.edu/dept/dial/ } \\
\hline E-Mail & \multicolumn{2}{|l|}{ Dial@dial.msstate.edu } \\
\hline $\begin{array}{l}\text { Services Available } \\
\text { (Select all that apply) }\end{array}$ & $\begin{array}{ll}\text { O } & \text { Service provider } \\
- & \text { Sells technology model }\end{array}$ & $\begin{array}{ll} & \text { Rents technology model } \\
\text { O Will train site users, \# hours : } \\
\text { hours }\end{array}$ \\
\hline References & \multicolumn{2}{|c|}{$\begin{array}{l}\text { Henderson, Mark E., December 1997, "Fourier Transform Profilometry with } \\
\text { Code in Matlab," Masters Thesis, Department of Electrical and Computer } \\
\text { Engineering, Mississippi State University. }\end{array}$} \\
\hline Publications & \multicolumn{2}{|l|}{ NA } \\
\hline
\end{tabular}


Appendix A. FTP Demonstration Summary (continued)

\begin{tabular}{|c|c|}
\hline \multicolumn{2}{|c|}{ VENDOR SUPPLIED INFORMATION } \\
\hline $\begin{array}{l}\text { Required Personnel for } \\
\text { Operation }\end{array}$ & $\begin{array}{l}\text { State number of each required for basic operation of equipment: } \\
\text { Equipment operators, } \\
\text { O Technicians, } \\
\text { O NEW: }\end{array}$ \\
\hline $\begin{array}{l}\text { Technology Model } \\
\text { Availability }\end{array}$ & Prototype (Technology is still in development) \\
\hline $\begin{array}{l}\text { Maintenance } \\
\text { Requirements }\end{array}$ & $\begin{array}{l}\text { The rail used to support the projector and camera needs occasional de- } \\
\text { spurring to account for nicks in the aluminum. No other maintenance is } \\
\text { performed on a regular basis. }\end{array}$ \\
\hline $\begin{array}{l}\text { Total Maintenance Cost } \\
\text { per } \mathrm{ft}^{2}\left(\$ / \mathrm{ft}^{2}\right)\end{array}$ & N/A \\
\hline $\begin{array}{l}\text { Technology Support } \\
\text { Equipment and Cost for } \\
\text { Each Unit (\$) }\end{array}$ & $\begin{array}{l}\text { Curtain: } \$ 20-\$ 100 \text { (If technology is used during daylight and price varies } \\
\text { with material) }\end{array}$ \\
\hline $\begin{array}{l}\text { Consumables and Cost for } \\
\text { Each Unit (\$) }\end{array}$ & $\begin{array}{l}\text { Projector light source (Bulbs rated at } \sim 500 \text { hours): } \$ 35 \text { (1998) } \\
\text { - Batteries - 4AA - Last } \sim 50 \text { images (only used in places where power is } \\
\text { limited): } \$ 2(1998) \\
\text { Rechargeable NI-Cad Battery for projector ( } \sim 5000 \text { measurements): } \$ 50 \\
\text { (1998) }\end{array}$ \\
\hline
\end{tabular}




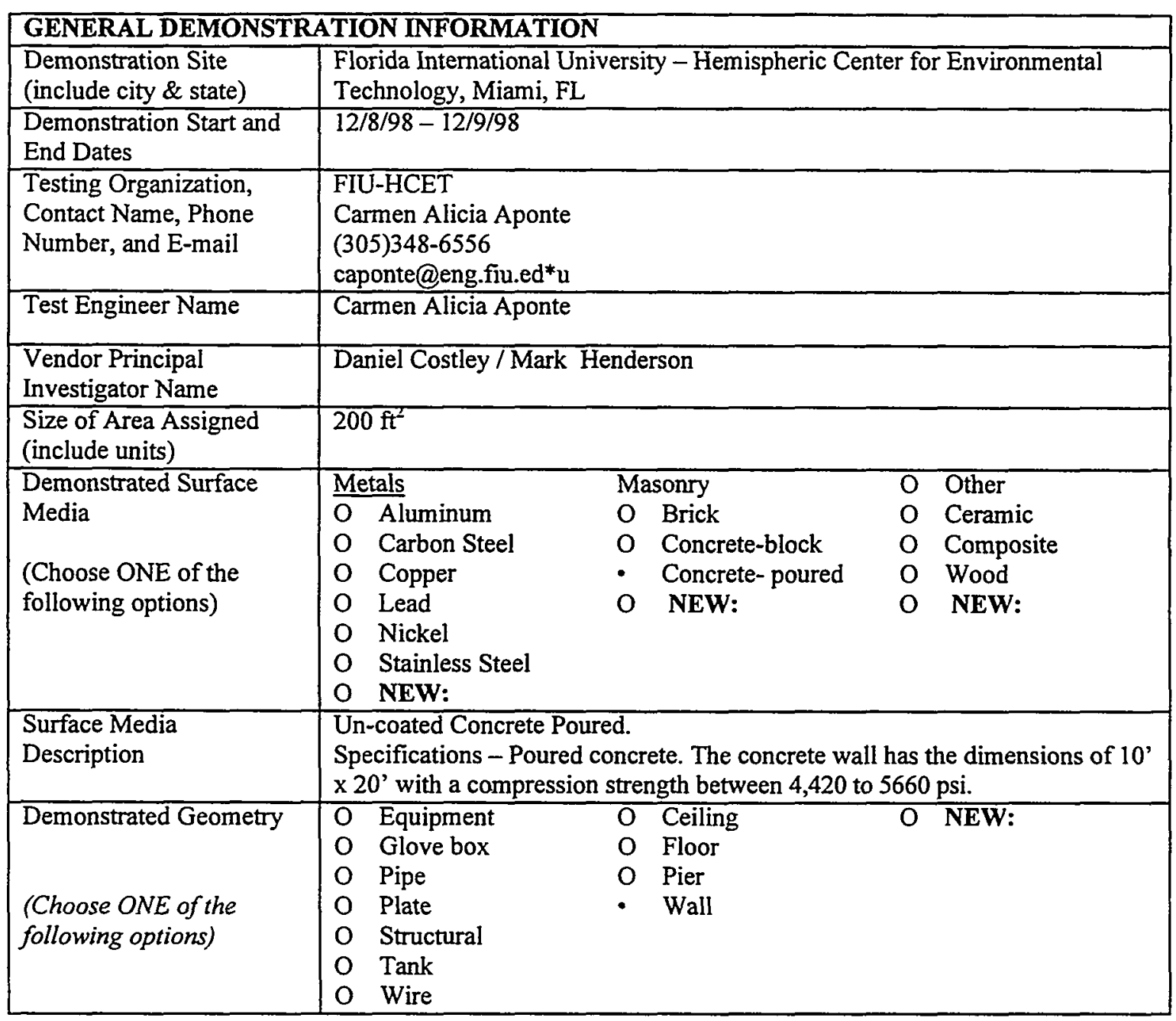


Appendix A. FTP Demonstration Summary (continued)

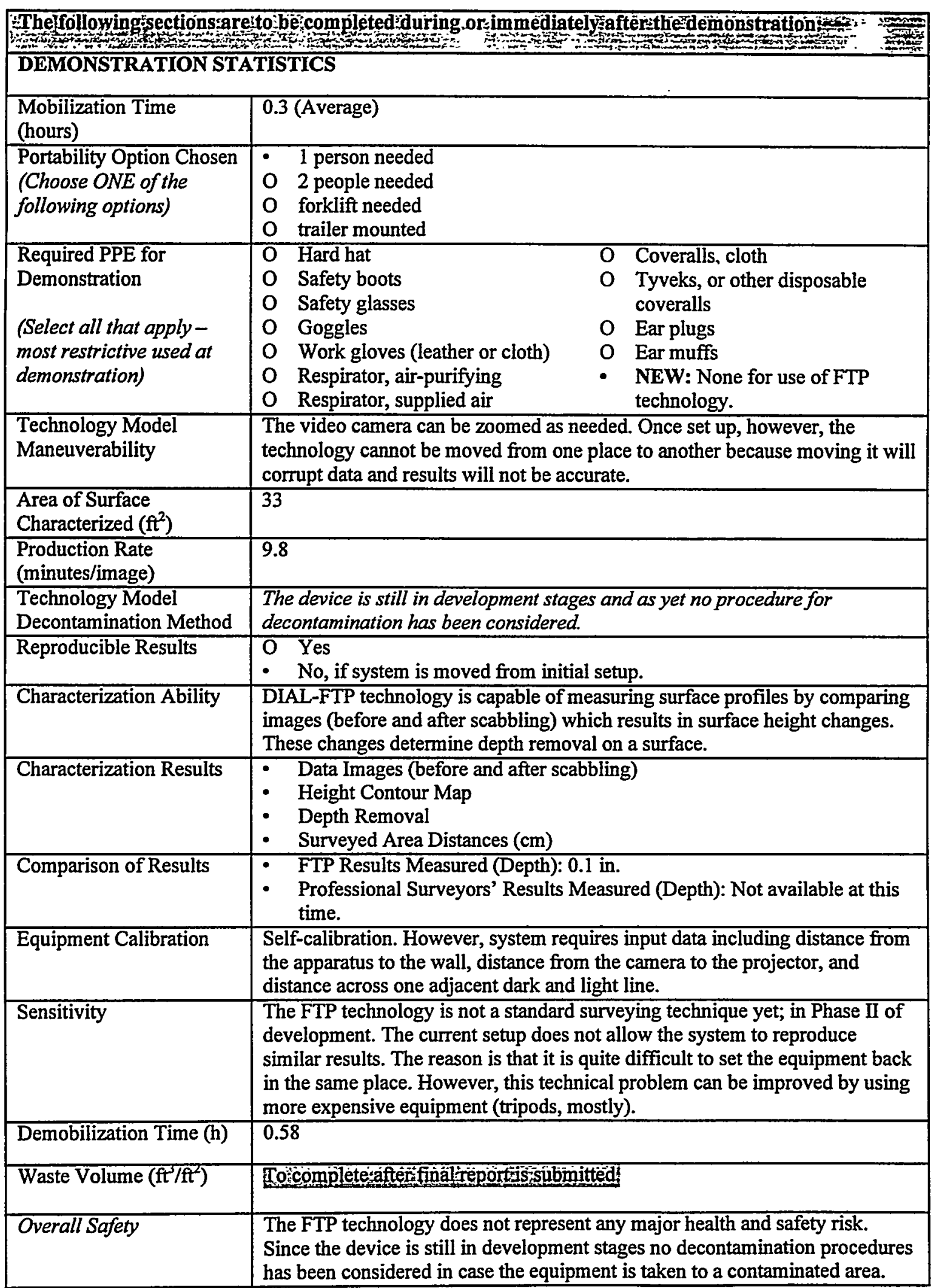


Appendix A. FTP Demonstration Summary (continued)

\begin{tabular}{|l|l|}
\hline OVERALL RATING OF TECHNOLOGY \\
\hline Effectiveness & $\begin{array}{l}\text { Circle rating: (low) } 1 \text { (high) } \\
\text { Reason for rating: Even though DIÄL-FTP is currently in testing phase, it is a } \\
\text { potentially useful technology in D\&D activities. The feedback time is } \\
\text { relatively short; thus, critical decisions regarding surface depth can be made } \\
\text { during the removal process. However, since the projection power is relatively } \\
\text { low, the lighting conditions in the area will make a difference in whether the } \\
\text { light pattern projection will be visible on the surface in question. }\end{array}$ \\
\hline $\begin{array}{l}\text { FTP technology can process images in relatively short periods of time (3 } \\
\text { minutes average), thus, feedback information is quick. }\end{array}$ \\
$\begin{array}{ll}\text { Setup, self-calibration equipment, and input data are easy and rapid (30 } \\
\text { minutes average) } \\
\text { The FTP equipment is light. No major physical operator effort is needed } \\
\text { to maneuver the system. } \\
\text { The system is user-friendly, and no major training is needed to operate it. }\end{array}$ \\
\hline Limitations & $\begin{array}{l}\text { The system cannot take images in daylight. The sunlight interferes with } \\
\text { the projector and light patterns are not visible; thus images cannot be } \\
\text { taken. } \\
\text { If images are taken during daylight, the area surveyed has to be shaded } \\
\text { until grid lines become visible. } \\
\text { Data can be corrupted if the apparatus is accidentally moved from initial } \\
\text { position. Therefore, data measurements may not be accurate. } \\
\text { The monitor must have a clear view of the wall. Concrete removal } \\
\text { technologies that produce dust while decontamination is ongoing cannot } \\
\text { be survey. }\end{array}$ \\
\hline Data Sensitivities & $\begin{array}{l}\text { The FTP is a prototype technology. The demonstration done at FIU-HCET } \\
\text { was the first one in the field. Real-world problems were accounted such as } \\
\text { pattern visibility difficulties during daylight. Therefore, reference images } \\
\text { were taken before sunrise or after sunset. This aspect can affect images' } \\
\text { accuracy due to projection light measurements. } \\
\text { The data collected on the first day was corrupted during the day. The } \\
\text { surveyors explained that it happens when the apparatus is accidentally moved } \\
\text { from initial position. }\end{array}$ \\
\hline
\end{tabular}


DECONTAMINATION TECHNOLOGIES FOR BUILDING MATERIALS

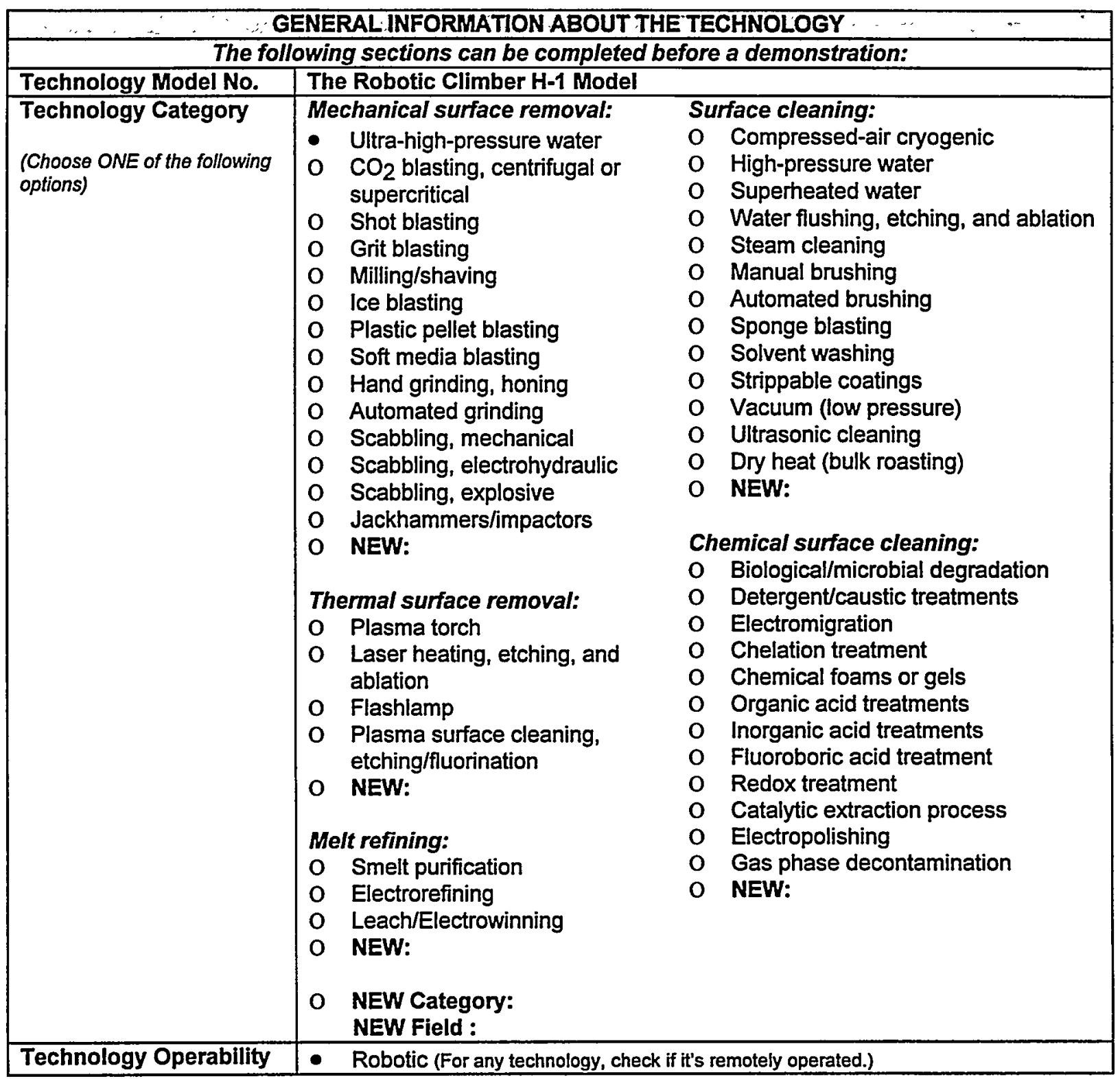

Page 1 of 19

01/22/99 Revision 1 
DECONTAMINATION TECHNOLOGIES FOR BUILDING MATERIALS

(Continued)

\begin{tabular}{|c|c|}
\hline \multicolumn{2}{|r|}{ GENERAL INFORMATION ABOUT THE TECHNOLOGY } \\
\hline $\begin{array}{l}\text { Technology Model } \\
\text { Description } \\
\text { (Attach separate sheet if } \\
\text { necessary) }\end{array}$ & $\begin{array}{l}\text { A remote-controlled, free-climbing robot using Ultra High Pressure Water } \\
\text { Jetting within a contained vacuum shroud. This technology employs a self- } \\
\text { propelled, joy-stick-operated robotic device designated for surface } \\
\text { decontamination, coating removal, and concrete "scabbling." Variable water } \\
\text { pressure ( }(0 \text { to } 36,000 \text { PSI) at low consumption rates is the medium used by the } \\
\text { H-1 model. } \\
\text { Adhesion to the surface is achieved using vacuum, enabling the robot to } \\
\text { perform on vertical or inverted surfaces as well as horizontal flats and sloped } \\
\text { surfaces. This vacuum also serves to capture the water from the removal } \\
\text { process as well as the waste. Once captured, the waste/water is transported to } \\
\text { a holding tank for future treatment and processing. The Robotic Climber is a } \\
\text { work platform given mobility with two tank-like tracks powered by two } \\
\text { independent motors. An Ultra High Pressure rotary nozzle with eight spray tips } \\
\text { housed beneath the robot spins at high speed, delivering the water jet to the } \\
\text { surface, cutting an 8-inch path. A vacuum chamber houses the spray tips and is } \\
\text { encircled with a seal that holds the robot to the surface and prevents the egress } \\
\text { of water and waste into the environment. } \\
\text { Dimension of technology model (L } \times W \times H): 2^{\prime} \times 2 \text { ' } \times 18^{n} \text {. } \\
\text { The weight of the technology model (lb): } 67 \text {. }\end{array}$ \\
\hline $\begin{array}{l}\text { Technology Model } \\
\text { Capital Cost (\$) } \\
\text { (Not Inc. Support Equip.) }\end{array}$ & $\begin{array}{r}\text { Robotic Climber Base System: Monthly rental - } \$ 36,000 \\
\text { Weekly rental - } \$ 12,500 \\
\text { Robotic Climber Hydro Blast System (36,000 psi): } \\
\text { Monthly rental - } \$ 60,000 \\
\text { Weekly rental - } \$ 20,800\end{array}$ \\
\hline $\begin{array}{l}\text { Technology Model Cost } \\
\text { Year (Year Only) }\end{array}$ & 1998 \\
\hline $\begin{array}{l}\text { Technology Model } \\
\text { Availability } \\
\text { (Choose ONE of the following } \\
\text { options.) }\end{array}$ & $\begin{array}{llll} & \text { Available off the shelf } & \text { O } & \text { 8-10 week turnaround } \\
\text { O } & 2-3 \text { week turnaround } & 0 & 6 \text { month turnaround } \\
\text { O } & 4-7 \text { week turnaround } & 0 & >6 \text { month turnaround } \\
& & & \text { Specify when: }\end{array}$ \\
\hline $\begin{array}{l}\text { Maturity of Technology } \\
\text { (Choose ONE of the following } \\
\text { options) }\end{array}$ & $\begin{array}{l}\text { - } \text { Commercially available } \\
\text { O Prototype } \\
\text { O NEW: }\end{array}$ \\
\hline $\begin{array}{l}\text { Useful Life Expectancy } \\
\text { (hour(s) ONLV }\end{array}$ & $\geq 5$ years (Assume hours per year) Rléaseiprovidê \\
\hline $\begin{array}{l}\text { Utility Requirements for } \\
\text { Technology Model }\end{array}$ & $\begin{array}{l}\text { Water supply }-6 \mathrm{gpm} \text { at } 30 \mathrm{psi} \text {. } \\
\text { Electricity }-110 \mathrm{~V} \text { for filtration system. } \\
\text { Diesel fuel for air compressor and water pump. } \\
\text { Gasoline for vacuum }\end{array}$ \\
\hline
\end{tabular}

Page 2 of 19 
DECONTAMINATION TECHNOLOGIES FOR BUILDING MATERIALS

(Continued)

\begin{tabular}{|c|c|c|c|c|}
\hline \multicolumn{5}{|c|}{ GENERAL INFORMATION ABOUT THE'TECHNOLOGY } \\
\hline $\begin{array}{l}\text { Applicable Surface } \\
\text { Media } \\
\text { (Select all that apply) }\end{array}$ & $\begin{array}{l}\text { Metals: } \\
\text { - Aluminum } \\
\text { - Carbon steel } \\
\text { - Copper } \\
\text { - Lead } \\
\text { - Nickel } \\
\text { - Stainless steel } \\
\text { O NEW: }\end{array}$ & $\begin{array}{l}\text { Mas } \\
\bullet \\
\bullet \\
\bullet \\
0\end{array}$ & -block & \begin{tabular}{ll}
\multicolumn{2}{l}{ Other: } \\
o & Ceramic \\
- & Composite \\
O & Wood \\
- NEW: Fiberglass
\end{tabular} \\
\hline $\begin{array}{l}\text { Applicable Geometries } \\
\text { (Select all that apply) }\end{array}$ & \multicolumn{2}{|l|}{$\begin{array}{ll}\text { O } & \text { Equipment } \\
\text { O } & \text { Glove box } \\
\text { O } & \text { Pipe } \\
\text { - } & \text { Plate } \\
\text { O } & \text { Structural } \\
\text { O } & \text { Tank } \\
\end{array}$} & \multicolumn{2}{|c|}{$\begin{array}{ll}\text { O } & \text { Wire } \\
\text { - Ceiling } \\
\text { - } & \text { Floor } \\
\text { - Pier } \\
\text { - Wall } \\
\text { O NEW: }\end{array}$} \\
\hline $\begin{array}{l}\text { Removal Capabilities } \\
\text { (Select all that apply) }\end{array}$ & \multicolumn{2}{|c|}{$\begin{array}{l}\text { Metals or Other: } \\
\text { - Surface cleaning } \\
\text { - Less than coating/rust } \\
\text { - Coating/rust removal } \\
\text { o NEW: } \\
\text { NEW Category: } \\
\text { NEW Field: }\end{array}$} & \multicolumn{2}{|c|}{$\begin{array}{l}\text { Masonry: } \\
\text { - Surface cleaning } \\
\text { - } \quad \text { Less than coating/rust } \\
\text { - } \text { Coating/rust removal } \\
\text { - Less than } 1 / 4 \text { in. } \\
\text { - Greater than } 1 / 4 \text { but less than } 1 / 2 \text { in. } \\
\text { O Greater than } 1 / 2 \text { in. but less than } 1 \text { in. } \\
\text { O Greater than } 1 \text { in. (cracks) } \\
\text { O NEW: }\end{array}$} \\
\hline $\begin{array}{l}\text { Portability Options } \\
\text { (Select all that apply) }\end{array}$ & \multicolumn{4}{|c|}{$\begin{array}{ll}\text { O } & 1 \text { person needed to remove from truck } \\
\text { o } & 2 \text { people needed to remove from truck } \\
\text { - } & \text { Forklift needed to remove from truck } \\
\text { - Truck/trailer mounted } \\
\text { O NEW: }\end{array}$} \\
\hline
\end{tabular}




\section{DECONTAMINATION TECHNOLOGIES FOR BUILDING MATERIALS \\ (Continued)}

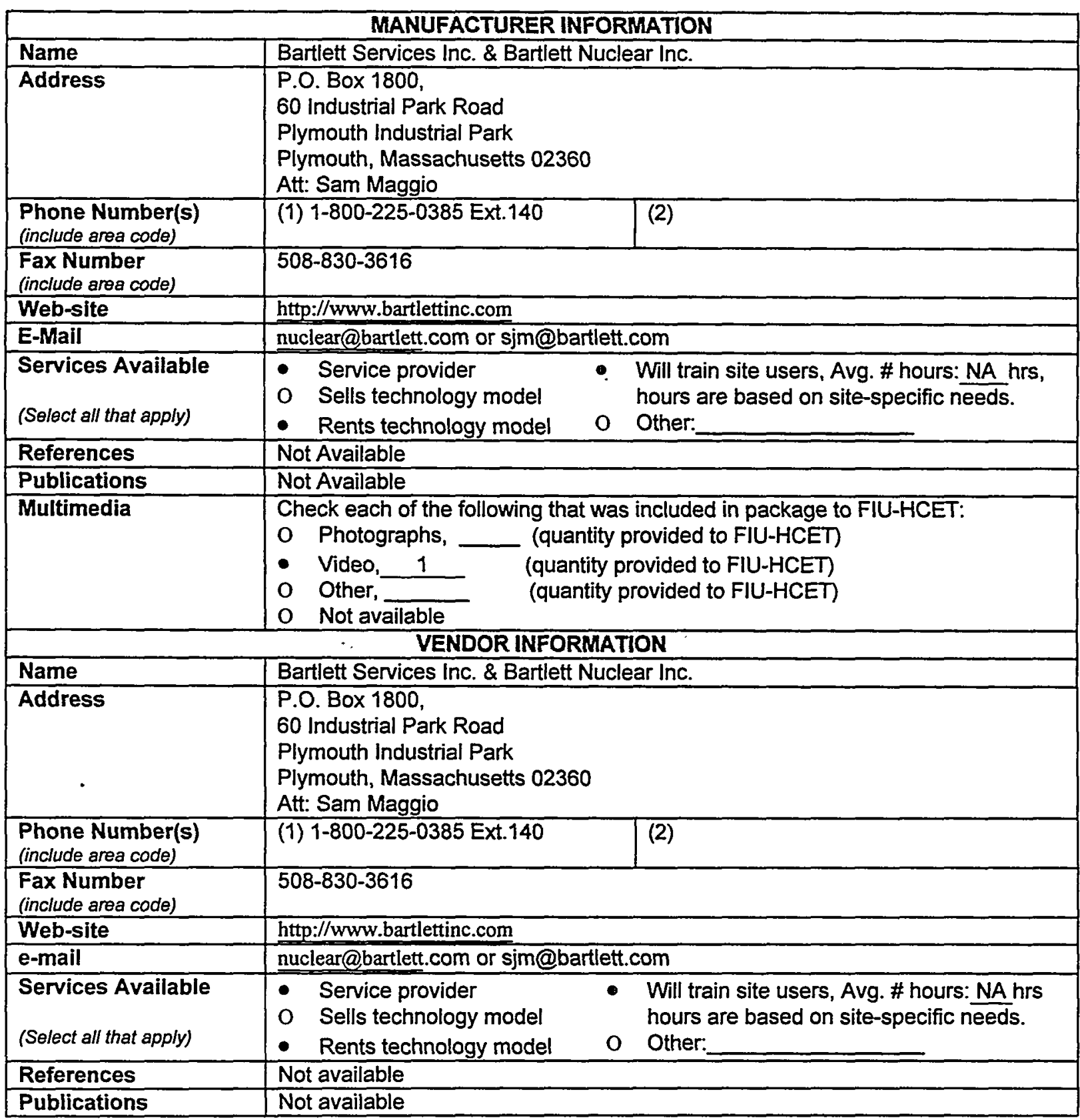


DECONTAMINATION TECHNOLOGIES FOR BUILDING MATERIALS

(Continued)

\begin{tabular}{|c|c|}
\hline & VENDOR SUPPLIED INFORMATION \\
\hline $\begin{array}{l}\text { Removal Media Type } \\
\text { and Cost (\$) per Unit }\end{array}$ & $\begin{array}{l}\text { Type: water } \\
\text { Cost: } \$ 0.7693 \\
\text { Unit: } 1,000 \text { gallons }\end{array}$ \\
\hline $\begin{array}{l}\text { Required Personnel } \\
\text { for Operation }\end{array}$ & $\begin{array}{l}\text { State number of each required for basic operation of equipment: } \\
\text { O Equipment operators, } \\
\text { O Technicians, } \\
\text { O NEW: }\end{array}$ \\
\hline $\begin{array}{l}\text { Scale-up } \\
\text { Requirements } \\
\text { (for } 10,000 \mathrm{ft}^{2} \text { or over) }\end{array}$ & Single basic unit with support equipment. \\
\hline $\begin{array}{l}\text { Maintenance } \\
\text { Requirements }\end{array}$ & $\begin{array}{l}\text { - } \text { Pump packings } \\
\text { - Spray tips } \\
\text { - } \text { Check: Hoses, seal rings, tank tracks, water filters }\end{array}$ \\
\hline $\begin{array}{l}\text { Total Maintenance } \\
\text { Cost per } \mathrm{ft}^{2}\left(\$ / \mathrm{ft}^{2}\right)\end{array}$ & $\$ 12,000-15,000$ per year (Seals, tracks and wear items) \\
\hline $\begin{array}{l}\text { Technology Support } \\
\text { Equipment and Cost } \\
\text { for Each Unit (\$) }\end{array}$ & $\begin{array}{l}\text { Pléase providejcost } \\
\text { - Ultra high pressure hydroblast pump- } 150 \mathrm{Hp} \\
\text { - Vacuum system }-450 \mathrm{cfm} \\
\text { - Air compressor }-185 \mathrm{~cm} \\
\text { - Safety tether system - Air-powered winch and cable to prevent accidental } \\
\text { falls. }\end{array}$ \\
\hline $\begin{array}{l}\text { Consumables } \\
\text { and Cost for } \\
\text { Each Unit (\$) }\end{array}$ & $\begin{array}{l}\text { Please provide } \\
\text { Seals - \$/unit } \\
\text { Spray tips - \$/unit } \\
\text { Hoses - \$/unit } \\
\text { Water filters - \$/unit } \\
\text { Etc. - \$/unit }\end{array}$ \\
\hline
\end{tabular}




\section{DECONTAMINATION TECHNOLOGIES FOR BUILDING MATERIALS (Continued)}

\begin{tabular}{|c|c|c|c|c|}
\hline \multicolumn{5}{|c|}{$\begin{array}{l}\text { DEMONSTRATION INFORMATION } \\
\text { Coated Metal Plates }\end{array}$} \\
\hline $\begin{array}{l}\text { Demonstration Site } \\
\text { (include city \& state) }\end{array}$ & \multicolumn{4}{|c|}{$\begin{array}{l}\text { Florida International University - Hemispheric Center for Environmental } \\
\text { Technology, Miami, FL }\end{array}$} \\
\hline Demonstration Date & \multicolumn{4}{|l|}{$\begin{array}{l}\text { Start: } 12 / 08 / 98 \\
\text { End: } 12 / 08 / 98\end{array}$} \\
\hline $\begin{array}{l}\text { Testing Organization } \\
\text { Information }\end{array}$ & \multicolumn{4}{|c|}{$\begin{array}{l}\text { Testing Organization: FIU-HCET } \\
\text { Contact Name: Cindy Zhang } \\
\text { Phone Number: (305) 348-6340 } \\
\text { e-mail: zhangc@eng.fiu.edu }\end{array}$} \\
\hline Vendor Name & \multicolumn{4}{|c|}{ Bartlett Services Inc. \& Bartlett Nuclear Inc. } \\
\hline Test Engineer Name & \multicolumn{4}{|c|}{\begin{tabular}{|l|l|l} 
Cindy Zhang \\
\end{tabular}} \\
\hline $\begin{array}{l}\text { Vendor Principal Investigator } \\
\text { Name }\end{array}$ & \multicolumn{4}{|l|}{ Sam Maggio } \\
\hline $\begin{array}{l}\text { Size of Area Assigned } \\
\text { (include units) }\end{array}$ & \multicolumn{4}{|l|}{$64 \mathrm{ft}^{2}$} \\
\hline $\begin{array}{l}\text { Demonstrated Surface Media } \\
\text { (Choose ONE of the following } \\
\text { options) }\end{array}$ & \multicolumn{4}{|c|}{$\begin{array}{ll}\text { Metals: } \\
\text { O } & \text { Aluminum } \\
\text { - } & \text { Carbon steel } \\
\text { O } & \text { Copper } \\
\text { O } & \text { Lead } \\
\text { O } & \text { Nickel } \\
\text { O } & \text { Stainless steel } \\
\text { O } & \text { NEW: }\end{array}$} \\
\hline Surface Media Description & \multicolumn{4}{|c|}{$\begin{array}{l}\text { Coated metal plates with an epoxy polyamine coating primer of } 7 \text { mils Ply- } \\
\text { Mastic and } 1.5 \text { mils Ply-Thane } 890 \mathrm{HS} .1 / 4-\text { in. thick }\end{array}$} \\
\hline $\begin{array}{l}\text { Demonstrated Geometry } \\
\text { (Choose ONE of the following } \\
\text { options) }\end{array}$ & $\begin{array}{|ll|}\text { O } & \text { Equipment } \\
\text { O } & \text { Glove box } \\
\text { O } & \text { Pipe } \\
\text { - } & \text { Plate } \\
0 & \text { Structural } \\
\text { O } & \text { Tank } \\
\text { O } & \text { Wire } \\
\end{array}$ & $\begin{array}{ll}\text { O } & \text { Ceilin } \\
\text { O } & \text { Floor } \\
\text { O } & \text { Pier } \\
\text { O } & \text { Wall } \\
\text { O } & \text { NEW }\end{array}$ & & \\
\hline \multicolumn{5}{|c|}{ The following sections are to be completed during or immediately after the demonstration: } \\
\hline Mobilization Time (h) & \multicolumn{4}{|l|}{4.33} \\
\hline $\begin{array}{l}\text { Portability Option Chosen } \\
\text { (Choose ONE of the following } \\
\text { options) }\end{array}$ & \multicolumn{4}{|c|}{$\begin{array}{ll}\mathrm{O} & 1 \text { person needed } \\
\mathrm{O} & 2 \text { people needed } \\
- & \text { forklift needed } \\
\mathrm{O} & \text { trailer mounted } \\
\mathrm{O} & \text { Not available } \\
\mathrm{O} & \text { NEW: }\end{array}$} \\
\hline $\begin{array}{l}\text { Required PPE for } \\
\text { Demonstration } \\
\text { (Select all that apply- most } \\
\text { restrictive used at demonstration) }\end{array}$ & \multicolumn{2}{|c|}{$\begin{array}{ll}\text { - } & \text { Hard hat } \\
\text { - } & \text { Safety boots } \\
\text { - } & \text { Safety glasses } \\
\text { O } & \text { Goggles } \\
\text { O Work gloves (leather or cloth) } \\
\text { O Respirator, air-purifying } \\
\text { O } & \text { Respirator, supplied air }\end{array}$} & \multicolumn{2}{|c|}{$\begin{array}{l}\text { Coveralls, cloth } \\
\text { Tyveks, or other disposable } \\
\text { coveralls } \\
\text { Ear plugs } \\
\text { Ear muffs } \\
\text { Not available } \\
\text { NEW: }\end{array}$} \\
\hline $\begin{array}{l}\text { Technology Model } \\
\text { Maneuverability }\end{array}$ & \multicolumn{4}{|c|}{$\begin{array}{l}\text { Robot is remotely operated by joysticks. This allows the robot to move in } \\
\text { any direction. }\end{array}$} \\
\hline
\end{tabular}




\section{DECONTAMINATION TECHNOLOGIES FOR BUILDING MATERIALS}

(Continued)

\begin{tabular}{|c|c|c|c|}
\hline \multicolumn{4}{|c|}{$\begin{array}{l}\text { DEMONSTRATION INFORMATION } \\
\text { Coated Metal Plates }\end{array}$} \\
\hline $\begin{array}{l}\text { Area of Surface } \\
\text { Decontaminated }\left(\mathrm{ft}^{2}\right)\end{array}$ & \multicolumn{3}{|c|}{ 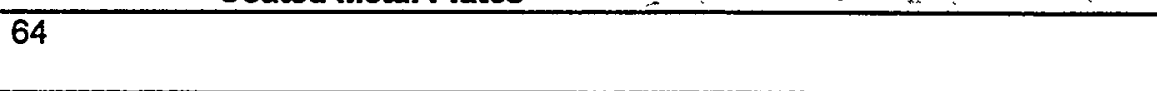 } \\
\hline Production Rate (ft'/h) & \multicolumn{3}{|l|}{73.56} \\
\hline $\begin{array}{l}\text { Depth of Surface } \\
\text { Removed and Method for } \\
\text { Determining Depth }\end{array}$ & \multicolumn{2}{|c|}{$\begin{array}{ll}\text { O } & \text { Surface cleaning } \\
\text { O } & \text { Less than coating/rust } \\
\text { - } & \text { Coating/rust removal } \\
\text { O } & \text { Less than } 1 / 4 \text { in. } \\
\text { O } & \text { Greater than } 1 / 4 \text { but less than } 1 / 2 \text { in. } \\
\text { O } & \text { Greater than } 1 / 2 \text { in. but less than } 1 \text { in. } \\
\text { O } & \text { Greater than } 1 \text { in. (cracks) }\end{array}$} & $\begin{array}{l}\text { d: } \\
\text { Juantity: } \quad \text { (in.) } \\
\text { fuantity: } \quad \text { (in.) } \\
\text { uantity: } \quad \text { (in.) }\end{array}$ \\
\hline Removal Gap (in.) & \multicolumn{3}{|c|}{$\begin{array}{l}0 \\
0\end{array}$ Greater than 1 in. (cracks) $\quad$ Exact quantity: } \\
\hline $\begin{array}{l}\text { Removal Media Usage } \\
\text { Rate (Unit/ft') }\end{array}$ & \multicolumn{3}{|l|}{$\begin{array}{l}\text { Type: Water } \\
\text { Quantity: } 4.31 \\
\text { Unit: gpm }\end{array}$} \\
\hline $\begin{array}{l}\text { Technology Model } \\
\text { Decontamination Method }\end{array}$ & \multicolumn{3}{|l|}{ Please:provide } \\
\hline Demobilization Time (h) & \multicolumn{3}{|l|}{0.73} \\
\hline $\begin{array}{l}\text { Ending Surface } \\
\text { Condition } \\
\text { (Choose ONE of the following } \\
\text { options) }\end{array}$ & $\begin{array}{ll}\text { Metal (Steel): } \\
\text { O } \\
\text { O Comsh-off finish } \\
\text { O Near white metal } \\
\text { O finish } \\
\text { - White metal finish } \\
\text { O NEW: }\end{array}$ & $\begin{array}{ll}\text { Metal (Other): } \\
\text { o } & \text { Coating/rust not } \\
\text { successfully } \\
\text { removed } \\
\text { o Dull bare metal } \\
\text { finish } \\
\text { O Shiny bare metal } \\
\text { finish } \\
\text { O NEW: }\end{array}$ & $\begin{array}{ll}\text { Masonry and Other: } \\
\text { o Coating not successfully } \\
\text { removed } \\
\text { o Coating removed } \\
\text { o Rough, bare, uneven } \\
\text { surface } \\
\text { o Smooth, bare, even } \\
\text { surface } \\
\text { o NEW: }\end{array}$ \\
\hline $\begin{array}{l}\left.\text { Waste Volume ( } \mathrm{ft}^{3} / \mathrm{ft}^{2}\right) \\
\text { (include only substrate removed } \\
\text { and spent media) }\end{array}$ & \multicolumn{3}{|l|}{0.473} \\
\hline $\begin{array}{l}\text { Waste Characteristics } \\
\text { (include only substrate removed } \\
\text { and spent media) }\end{array}$ & \multicolumn{3}{|c|}{ Water with suspended paint particles. } \\
\hline
\end{tabular}


DECONTAMINATION TECHNOLOGIES FOR BUILDING MATERIALS

(Continued)

\begin{tabular}{|c|c|c|c|c|c|}
\hline \multicolumn{6}{|c|}{$\begin{array}{l}\text { DEMONSTRATION INFORMATION } \\
\text { Rusted Metal Plates }\end{array}$} \\
\hline $\begin{array}{l}\text { Demonstration Site } \\
\text { (include city \& state) }\end{array}$ & \multicolumn{5}{|c|}{$\begin{array}{l}\text { Florida International University - Hemispheric Center for Environmental } \\
\text { Technology, Miami, FL }\end{array}$} \\
\hline Demonstration Date & \multicolumn{5}{|c|}{$\begin{array}{l}\text { Start: } 12 / 08 / 98 \\
\text { End: } 12 / 08 / 98\end{array}$} \\
\hline $\begin{array}{l}\text { Testing Organization } \\
\text { Information }\end{array}$ & \multicolumn{5}{|c|}{$\begin{array}{l}\text { Testing Organization: FIU-HCET } \\
\text { Contact Name: Cindy Zhang } \\
\text { Phone Number: (305) 348-6340 } \\
\text { e-mail: zhangc@eng.fiu.edu }\end{array}$} \\
\hline Vendor Name & \multicolumn{5}{|c|}{ Bartlett Services Inc. \& Bartlett Nuclear Inc. } \\
\hline Test Engineer Name & \multicolumn{5}{|c|}{ Cindy Zhang } \\
\hline $\begin{array}{l}\text { Vendor Principal } \\
\text { Investigator Name }\end{array}$ & \multicolumn{5}{|c|}{ Sam Maggio } \\
\hline $\begin{array}{l}\text { Size of Area Assigned } \\
\text { (include units) }\end{array}$ & \multicolumn{5}{|c|}{$64 \mathrm{ft}^{2}$} \\
\hline $\begin{array}{l}\text { Demonstrated Surface } \\
\text { Media } \\
\text { (Choose ONE of the following } \\
\text { options) }\end{array}$ & \multicolumn{4}{|c|}{\begin{tabular}{|ll} 
Metals: \\
O & Aluminum \\
- & Carbon steel \\
O & Copper \\
O & Lead \\
0 & Nickel \\
O & Stainless steel \\
O & NEW: \\
\end{tabular}} & $\begin{array}{l}\text { Other: } \\
\text { O Ceramic } \\
\text { O Composite } \\
\text { O Wood } \\
\text { O NEW: }\end{array}$ \\
\hline $\begin{array}{l}\text { Surface Media } \\
\text { Description }\end{array}$ & \multicolumn{5}{|c|}{ Rusted steel plate. $1 / 1$-in. thick. } \\
\hline $\begin{array}{l}\text { Demonstrated Geometry } \\
\text { (Choose ONE of the following } \\
\text { options) }\end{array}$ & \multicolumn{2}{|r|}{$\begin{array}{|ll|}\text { O } & \text { Equipment } \\
\text { O } & \text { Glove box } \\
\text { O } & \text { Pipe } \\
\text { - } & \text { Plate } \\
\text { O } & \text { Structural } \\
\text { O } & \text { Tank } \\
\text { O } & \text { Wire } \\
\end{array}$} & \multicolumn{3}{|c|}{$\begin{array}{ll}\text { O } & \text { Ceiling } \\
\text { O } & \text { Floor } \\
\text { O } & \text { Pier } \\
\text { O } & \text { Wall } \\
\text { O } & \text { NEW: }\end{array}$} \\
\hline \multicolumn{6}{|c|}{ The following sections are to be completed during or immediately after the demonstration: } \\
\hline Mobilization Time (h) & \multicolumn{5}{|c|}{4.33} \\
\hline $\begin{array}{l}\text { Portability Option } \\
\text { Chosen } \\
\text { (Choose ONE of the } \\
\text { following options) }\end{array}$ & \multicolumn{5}{|c|}{$\begin{array}{ll}\text { O } & 1 \text { person needed } \\
0 & 2 \text { people needed } \\
\text { - } & \text { forklift needed } \\
\text { O } & \text { trailer mounted } \\
O & \text { Not available } \\
\text { O } & \text { NEW: } \\
\end{array}$} \\
\hline $\begin{array}{l}\text { Required PPE for } \\
\text { Demonstration } \\
\text { (Select all that apply-most } \\
\text { restrictive used at } \\
\text { demonstration) }\end{array}$ & \multicolumn{3}{|c|}{$\begin{array}{ll}\text { - } & \text { Hard hat } \\
\text { - } & \text { Safety boots } \\
\text { - } & \text { Safety glasses } \\
\text { o } & \text { Goggles } \\
\text { O Work gloves (leather or cloth) } \\
\text { o Respirator, air-purifying } \\
\text { o Respirator, supplied air }\end{array}$} & \multicolumn{2}{|r|}{ ralls } \\
\hline
\end{tabular}




\section{DECONTAMINATION TECHNOLOGIES FOR BUILDING MATERIALS (Continued)}

\begin{tabular}{|c|c|c|c|}
\hline \multicolumn{4}{|c|}{$\begin{array}{l}\text { DEMONSTRATIONINFORMATION } \\
\text { Rusted Metal Plates. }\end{array}$} \\
\hline $\begin{array}{l}\text { Technology Model } \\
\text { Maneuverability }\end{array}$ & \multicolumn{3}{|c|}{$\begin{array}{l}\text { Robot is remotely operated by joysticks. This allows the robot to move in any } \\
\text { direction. }\end{array}$} \\
\hline $\begin{array}{l}\text { Area of Surface } \\
\text { Decontaminated }\left(\mathrm{ft}^{2}\right)\end{array}$ & \multicolumn{3}{|l|}{$\overline{64}$} \\
\hline Production Rate $\left(\mathrm{ft}^{2} / \mathrm{h}\right)$ & \multicolumn{3}{|l|}{172.97} \\
\hline $\begin{array}{l}\text { Depth of Surface } \\
\text { Removed and Method for } \\
\text { Determining Depth }\end{array}$ & \multicolumn{2}{|c|}{$\begin{array}{ll}\text { O } & \text { Surface cleaning } \\
\text { O } & \text { Less than coating/rust } \\
\text { - } & \text { Coating/rust removal } \\
\text { O } & \text { Less than } 1 / 4 \text { in. } \\
\text { O } & \text { Greater than } 1 / 4 \text { but less than } 1 / 2 \text { in. } \\
\text { O } & \text { Greater than } 1 / 2 \text { in. but less than } 1 \text { in. } \\
\text { O } & \text { Greater than } 1 \text { in. (cracks) }\end{array}$} & $\begin{array}{l}\text { uantity: } \\
\text { uantity: } \\
\text { uantity: } \\
\text { Jantity: }\end{array}$ \\
\hline Removal Gap (in.) & \multicolumn{3}{|c|}{0} \\
\hline $\begin{array}{l}\text { Removal Media Usage } \\
\text { Rate (Unit/ft²) }\end{array}$ & \multicolumn{3}{|l|}{$\begin{array}{l}\text { Type: Water } \\
\text { Quantity: } 4.31 \\
\text { Unit: gpm }\end{array}$} \\
\hline \multicolumn{4}{|l|}{$\begin{array}{l}\text { Technology Model } \\
\text { Decontamination Method }\end{array}$} \\
\hline Demobilization Time (h) & \multicolumn{3}{|l|}{0.73} \\
\hline $\begin{array}{l}\text { Ending Surface } \\
\text { Condition } \\
\text { (Choose ONE of the following } \\
\text { options) }\end{array}$ & $\begin{array}{l}\text { Metal (Steel): } \\
\text { o Brush-off finish } \\
\text { o Commercial finish } \\
\text { o Near white metal } \\
\text { finish } \\
\text { - White metal finish } \\
\text { O NEW: } \\
\\
\text { New Category: } \\
\text { New Field: }\end{array}$ & $\begin{array}{ll}\text { Metal (Other): } \\
\text { O Coating/rust not } \\
\\
\text { successfully } \\
\text { removed } \\
\text { o } \\
\text { Dull bare metal } \\
\text { finish } \\
\text { o }\end{array}$ & $\begin{array}{ll}\text { Masonry and Other: } \\
\text { O Coating not } \\
\text { successfully removed } \\
\text { o Coating removed } \\
\text { O Rough, bare, uneven } \\
\text { surface } \\
\text { o } \\
\text { Smooth, bare, even } \\
\text { surface } \\
\text { O NEW: }\end{array}$ \\
\hline $\begin{array}{l}\text { Waste Volume (ft'/ft') } \\
\text { (include only substrate removed } \\
\text { and spent media) }\end{array}$ & 0.201 & & \\
\hline $\begin{array}{l}\text { Waste Characteristics } \\
\text { (include only substrate removed } \\
\text { and spent media) }\end{array}$ & Water with suspended & Ist particles. & \\
\hline
\end{tabular}




\section{DECONTAMINATION TECHNOLOGIES FOR BUILDING MATERIALS (Continued)}

\begin{tabular}{|c|c|c|c|}
\hline \multicolumn{4}{|c|}{$\begin{array}{l}\text { DEMONSTRATION INFORMATION } \\
\text { Coated Concrete Wall }\end{array}$} \\
\hline $\begin{array}{l}\text { Technology Model } \\
\text { Maneuverability }\end{array}$ & \multicolumn{3}{|c|}{$\begin{array}{l}\text { Robot is remotely operated by joysticks. This allows the robot to move in } \\
\text { any direction. }\end{array}$} \\
\hline $\begin{array}{l}\text { Area of Surface } \\
\text { Decontaminated }\left(\mathrm{ft}^{2}\right)\end{array}$ & \multicolumn{3}{|c|}{128.81} \\
\hline Production Rate $\left(\mathrm{ft}^{2} / \mathrm{h}\right)$ & \multicolumn{3}{|l|}{165.14} \\
\hline $\begin{array}{l}\text { Depth of Surface } \\
\text { Removed and Method } \\
\text { for Determining Depth }\end{array}$ & \multicolumn{3}{|c|}{$\begin{array}{lll}O & \text { Surface cleaning } & \text { NEW: } \\
0 & \text { Less than coating/rust } & \text { Method: } \\
- & \text { Coating/rust removal } & \\
0 & \text { Less than } 1 / 4 \text { in. } & \text { Exact quantity: } \\
0 & \text { Greater than } 1 / 4 \text { but less than } 1 / 2 \text { in. } & \text { Exact quantity:-(in.) } \\
0 & \text { Greater than } 1 / 2 \text { in. but less than } 1 \text { in. } & \text { Exact quantity: (in.) } \\
0 & \text { Greater than } 1 \text { in. (cracks) } & \text { Exact quantity: }\end{array}$} \\
\hline Removal Gap (in.) & \multicolumn{3}{|l|}{17.56} \\
\hline $\begin{array}{l}\text { Removal Media Usage } \\
\text { Rate (Unit } \mathrm{ft}^{2} \text { ) }\end{array}$ & \multicolumn{3}{|l|}{$\begin{array}{l}\text { Type: Water } \\
\text { Quantity: } 4.31 \\
\text { Unit: gpm } \\
\end{array}$} \\
\hline \multicolumn{4}{|l|}{$\begin{array}{l}\text { Technology Model } \\
\text { Decontamination } \\
\text { Method } \\
\end{array}$} \\
\hline Demobilization Time (h) & \multicolumn{3}{|l|}{0.73} \\
\hline $\begin{array}{l}\text { Ending Surface } \\
\text { Condition } \\
\text { (Choose ONE of the following } \\
\text { options) }\end{array}$ & $\begin{array}{ll}\text { Metal (Steel): } \\
0 & \text { Brush-off } \\
& \text { finish } \\
0 & \text { Commercial } \\
& \text { finish } \\
0 & \text { Near white } \\
& \text { metal finish } \\
0 & \text { White metal } \\
& \text { finish } \\
\text { O } & \text { NEW: } \\
& \\
\text { New Category: } \\
\text { New Field: }\end{array}$ & $\begin{array}{l}\text { Metal (Other): } \\
\text { O Coating/rust not } \\
\text { successfully } \\
\text { removed } \\
\text { O Dull bare metal } \\
\text { finish } \\
\text { O Shiny bare metal } \\
\text { finish } \\
\text { O NEW: }\end{array}$ & $\begin{array}{l}\text { Masonry and Other: } \\
\text { O Coating not successfully } \\
\text { removed } \\
\text { - Coating removed } \\
\text { O Rough, bare, uneven } \\
\text { surface } \\
\text { O Smooth, bare, even } \\
\text { surface } \\
\text { O NEW: }\end{array}$ \\
\hline $\begin{array}{l}\text { Waste Volume }\left(\mathrm{ft}^{3} / \mathrm{ft}^{2}\right) \\
\text { (include only substrate removed } \\
\text { and spent media) }\end{array}$ & 0.211 & & \\
\hline $\begin{array}{l}\text { Waste Characteristics } \\
\text { (include only substrate removed } \\
\text { and spent media) }\end{array}$ & Water with susp & d paint particles. & \\
\hline
\end{tabular}




\section{DECONTAMINATION TECHNOLOGIES FOR BUILDING MATERIALS (Continued)}

\begin{tabular}{|c|c|c|c|}
\hline \multicolumn{4}{|c|}{$\begin{array}{l}\text { DEMONSTRATION INFORMATION } \\
\text { Coated Floor }\end{array}$} \\
\hline $\begin{array}{l}\text { Demonstration Site } \\
\text { (include city \& state) }\end{array}$ & \multicolumn{3}{|c|}{$\begin{array}{l}\text { Florida International University - Hemispheric Center for Environmental } \\
\text { Technology, Miami, FL }\end{array}$} \\
\hline Demonstration Date & \multicolumn{3}{|c|}{$\begin{array}{l}\text { Start: } 12 / 10 / 98 \\
\text { End: } 12 / 10 / 98\end{array}$} \\
\hline $\begin{array}{l}\text { Testing Organization } \\
\text { Information }\end{array}$ & \multicolumn{3}{|c|}{$\begin{array}{l}\text { Testing Organization: FIU-HCET } \\
\text { Contact Name: Cindy Zhang } \\
\text { Phone Number: (305) 348-6340 } \\
\text { e-mail: zhangc@eng.fiu.edu }\end{array}$} \\
\hline Vendor Name & \multicolumn{3}{|c|}{ Bartlett Services Inc. \& Bartlett Nuclear Inc. } \\
\hline Test Engineer Name & \multicolumn{3}{|c|}{ Cindy Zhang } \\
\hline $\begin{array}{l}\text { Vendor Principal } \\
\text { Investigator Name }\end{array}$ & \multicolumn{3}{|l|}{ Sam Maggio } \\
\hline $\begin{array}{l}\text { Size of Area Assigned } \\
\text { (include units) }\end{array}$ & \multicolumn{3}{|l|}{$394.97 \mathrm{ft}^{2}$} \\
\hline $\begin{array}{l}\text { Demonstrated Surface } \\
\text { Media } \\
\text { (Choose ONE of the } \\
\text { following options) }\end{array}$ & $\begin{array}{ll}\text { Metals: } \\
\text { O } & \text { Aluminum } \\
\text { O } & \text { Carbon steel } \\
0 & \text { Copper } \\
\text { O } & \text { Lead } \\
\text { O } & \text { Nickel } \\
\text { O } & \text { Stainless steel } \\
\text { O } & \text { NEW: }\end{array}$ & $\begin{array}{ll}\text { Masonry: } \\
\text { O } & \text { Brick } \\
\text { O } & \text { Concrete-block } \\
\text { - } & \text { Concrete-poured } \\
\text { O } & \text { NEW: }\end{array}$ & $\begin{array}{ll}\text { Other: } \\
\text { O } & \text { Ceramic } \\
\text { O } & \text { Composite } \\
\text { O } & \text { Wood } \\
\text { O } & \text { NEW: }\end{array}$ \\
\hline $\begin{array}{l}\text { Surface Media } \\
\text { Description }\end{array}$ & \multicolumn{3}{|c|}{$\begin{array}{l}\text { Concrete - poured, with an epoxy polyamine coating primer of } 7 \text { mils Ply-Mastio } \\
\text { and } 1.5 \text { mils Ply-Thane } 890 \text { HS. The coated concrete floor has dimensions of } \\
236^{\prime \prime} \times 241^{\prime \prime} \text { with a compression strength between } 4,420 \text { to } 5,660 \text { psi. }\end{array}$} \\
\hline $\begin{array}{l}\text { Demonstrated } \\
\text { Geometry } \\
\text { (Choose ONE of the } \\
\text { following options) }\end{array}$ & \begin{tabular}{ll|}
0 & Equipment \\
0 & Glove box \\
O & Pipe \\
0 & Plate \\
0 & Structural \\
0 & Tank \\
0 & Wire \\
\end{tabular} & $\begin{array}{ll}\text { O } & \text { Ceiling } \\
\text { - } & \text { Floor } \\
\text { O } & \text { Pier } \\
\text { O } & \text { Wall } \\
\text { O } & \text { NEW: }\end{array}$ & \\
\hline \multicolumn{4}{|c|}{ The following sections are to be completed during or immediately after the demonstration: } \\
\hline Mobilization Time (h) & \multicolumn{3}{|l|}{4.33} \\
\hline $\begin{array}{l}\text { Portability Option } \\
\text { Chosen } \\
\text { (Choose ONE of the } \\
\text { following options) }\end{array}$ & \multicolumn{3}{|l|}{$\begin{array}{ll}\text { O } & 1 \text { person needed } \\
\text { O } & 2 \text { people needed } \\
\text { - forklift needed } \\
\text { o } & \text { trailer mounted } \\
\text { O } & \text { Not available } \\
0 & \text { NEW: } \\
\end{array}$} \\
\hline
\end{tabular}




\section{DECONTAMINATION TECHNOLOGIES FOR BUILDING MATERIALS (Continued)}

\begin{tabular}{|c|c|c|c|c|}
\hline \multicolumn{5}{|c|}{$\begin{array}{l}\text { DEMONSTRATION INFORMATION } \\
\text { Coated Floor }\end{array}$} \\
\hline $\begin{array}{l}\text { Required PPE for } \\
\text { Demonstration } \\
\text { (Select all that apply- } \\
\text { most restrictive used at } \\
\text { demonstration) }\end{array}$ & \multicolumn{4}{|c|}{$\begin{array}{lll}\text { - Hard hat } & \text { O } & \text { Coveralls, cloth } \\
\text { - Safety boots } & \text { O Tyveks, or other disposable } \\
\text { - Safety glasses } & & \text { coveralls } \\
\text { O Goggles } & \text { Ear plugs } \\
\text { O Work gloves (leather or } & \text { O Ear muffs } \\
& \text { Cloth) } & \text { O Not Available } \\
\text { O Respirator, air-purifying } & \text { O NEW: } \\
\text { O Respirator, supplied air } & & \end{array}$} \\
\hline $\begin{array}{l}\text { Technology Model } \\
\text { Maneuverability }\end{array}$ & \multicolumn{4}{|c|}{$\begin{array}{l}\text { Robot is remotely operated by joysticks. This allows the robot to move in } \\
\text { any direction. }\end{array}$} \\
\hline $\begin{array}{l}\text { Area of Surface } \\
\text { Decontaminated }\left(\mathrm{ft}^{2}\right)\end{array}$ & \multicolumn{4}{|c|}{341.30} \\
\hline Production Rate (ft'/h) & \multicolumn{4}{|l|}{235.38} \\
\hline $\begin{array}{l}\text { Depth of Surface } \\
\text { Removed and Method } \\
\text { for Determining Depth }\end{array}$ & \multicolumn{4}{|c|}{$\begin{array}{lll}\text { O } & \text { Surface cleaning } & \text { NEW: } \\
0 & \text { Less than coating/rust } & \text { Method: } \\
\text { - Coating/rust removal } & \\
0 & \text { Less than } 1 / 4 \text { in. } & \text { Exact quantity: } \\
0 & \text { Greater than } 1 / 4 \text { but less than } 1 / 2 \text { in. } & \text { Exact quantity:-(in.) } \\
0 & \text { Greater than } 1 / 2 \text { in. but less than } 1 \text { in. } & \text { Exact quantity:- (in.) } \\
0 & \text { Greater than } 1 \text { in. (cracks) } & \text { Exact quantity: }\end{array}$} \\
\hline Removal Gap (in.) & \multicolumn{4}{|c|}{8.88} \\
\hline $\begin{array}{l}\text { Removal Media Usage } \\
\text { Rate (Unit/ft²) }\end{array}$ & \multicolumn{4}{|l|}{$\begin{array}{l}\text { Type: Water } \\
\text { Quantity: } 4.31 \\
\text { Unit: gpm }\end{array}$} \\
\hline \multicolumn{5}{|l|}{$\begin{array}{l}\text { Technology Model } \\
\text { Decontamination } \\
\text { Method }\end{array}$} \\
\hline Demobilization Time (h) & \multicolumn{4}{|l|}{0.73} \\
\hline $\begin{array}{l}\text { Ending Surface } \\
\text { Condition } \\
\text { (Choose ONE of the following } \\
\text { options) }\end{array}$ & \begin{tabular}{ll}
\multicolumn{2}{l}{ Metal (Steel): } \\
O Brush-off \\
finish \\
o Commercial \\
finish \\
o Near white \\
metal finish \\
O White metal \\
finish \\
O NEW: \\
New Category: \\
New Field:
\end{tabular} & $\begin{array}{ll}\text { Metal } \\
\text { O } & \text { Coa } \\
& \text { succ } \\
& \text { rem } \\
0 & \text { Dull } \\
& \text { finis } \\
0 & \text { Shin } \\
& \text { finis } \\
0 & \text { NEV }\end{array}$ & $\begin{array}{l}\text { er): } \\
\text { lrust not } \\
\text { sfully } \\
\text { d } \\
\text { re metal } \\
\text { lare metal }\end{array}$ & $\begin{array}{l}\text { Masonry and Other: } \\
\text { O Coating not successfully } \\
\text { removed } \\
\text { - Coating removed } \\
\text { o Rough, bare, uneven } \\
\text { surface } \\
\text { o Smooth, bare, even } \\
\text { surface } \\
\text { o NEW: }\end{array}$ \\
\hline $\begin{array}{l}\text { Waste Volume }\left(\mathrm{ft}^{3} / \mathrm{ft}^{2}\right) \\
\text { (include only substrate removed } \\
\text { and spent media) }\end{array}$ & \multicolumn{4}{|l|}{0.148} \\
\hline $\begin{array}{l}\text { Waste Characteristics } \\
\text { (include only substrate removed } \\
\text { and spent media) }\end{array}$ & \multicolumn{4}{|c|}{ Water with suspended paint particles. } \\
\hline
\end{tabular}




\section{DECONTAMINATION TECHNOLOGIES FOR BUILDING MATERIALS (Continued)}

\begin{tabular}{|c|c|}
\hline \multicolumn{2}{|r|}{$\begin{array}{l}\text { DEMONSTRATION INFORMATION } \\
\text { Brick Wall }\end{array}$} \\
\hline $\begin{array}{l}\text { Demonstration Site } \\
\text { (include city \& state) }\end{array}$ & $\begin{array}{l}\text { Florida International University - Hemispheric Center for Environmental } \\
\text { Technology, Miami, FL }\end{array}$ \\
\hline Demonstration Date & $\begin{array}{l}\text { Start: } 12 / 11 / 98 \\
\text { End: } 12 / 11 / 98\end{array}$ \\
\hline $\begin{array}{l}\text { Testing Organization } \\
\text { Information }\end{array}$ & $\begin{array}{l}\text { Testing Organization: FIU-HCET } \\
\text { Contact Name: Cindy Zhang } \\
\text { Phone Number: (305) 348-6340 } \\
\text { e-mail: zhangc@eng.fiu.edu }\end{array}$ \\
\hline Vendor Name & Bartlett Services Inc. \& Bartlett Nuclear Inc. \\
\hline Test Engineer Name & Cindy Zhang \\
\hline $\begin{array}{l}\text { Vendor Principal } \\
\text { Investigator Name }\end{array}$ & Sam Maggio \\
\hline $\begin{array}{l}\text { Size of Area } \\
\text { Assigned } \\
\text { (include units) }\end{array}$ & $188.88 \mathrm{ft}^{2}$ \\
\hline $\begin{array}{l}\text { Demonstrated } \\
\text { Surface Media } \\
\text { (Choose ONE of the } \\
\text { following options) }\end{array}$ & 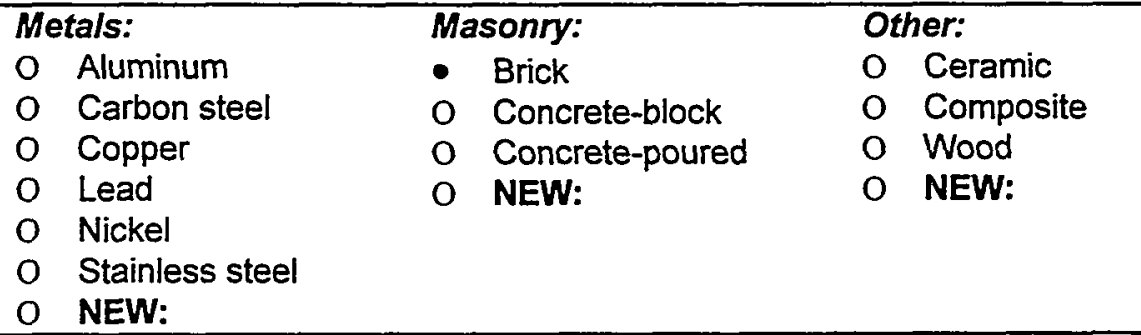 \\
\hline $\begin{array}{l}\text { Surface Media } \\
\text { Description }\end{array}$ & $\begin{array}{l}\text { Brick with an epoxy polyamine coating primer of } 7 \text { mils Ply-Mastic and } 1.5 \\
\text { mils Ply-Thane } 890 \mathrm{HS} \text {. Coated brick wall with the dimensions of } 230.5^{n} \times \\
118^{n} \text {. }\end{array}$ \\
\hline $\begin{array}{l}\text { Demonstrated } \\
\text { Geometry } \\
\text { (Choose ONE of the } \\
\text { following options) }\end{array}$ & $\begin{array}{llll}\text { O } & \text { Equipment } & \text { O } & \text { Ceiling } \\
\text { O } & \text { Glove box } & \text { O } & \text { Floor } \\
\text { O } & \text { Pipe } & \text { O } & \text { Pier } \\
\text { O } & \text { Plate } & \bullet & \text { Wall } \\
\text { O } & \text { Structural } & \text { O } & \text { NEW: } \\
\text { O } & \text { Tank } & & \\
\text { O } & \text { Wire } & & \\
\end{array}$ \\
\hline \multicolumn{2}{|c|}{ The following sections are to be completed during or immediately after the demonstration: } \\
\hline Mobilization Time (h) & 4.33 \\
\hline $\begin{array}{l}\text { Portability Option } \\
\text { Chosen } \\
\text { (Choose ONE of the } \\
\text { following options) }\end{array}$ & $\begin{array}{ll}0 & 1 \text { person needed } \\
0 & 2 \text { people needed } \\
\text { - } & \text { forklift needed } \\
0 & \text { trailer mounted } \\
0 & \text { Not available } \\
O & \text { NEW: } \\
\end{array}$ \\
\hline
\end{tabular}




\section{DECONTAMINATION TECHNOLOGIES FOR BUILDING MATERIALS}

(Continued)

\begin{tabular}{|c|c|c|c|c|}
\hline \multicolumn{5}{|c|}{$\begin{array}{l}\text { DEMONSTRATION INFORMATION } \\
\text { Brick Wall }\end{array}$} \\
\hline $\begin{array}{l}\text { Required PPE for } \\
\text { Demonstration } \\
\text { (Select all that apply-most } \\
\text { restrictive used at } \\
\text { demonstration) }\end{array}$ & \multicolumn{4}{|c|}{ 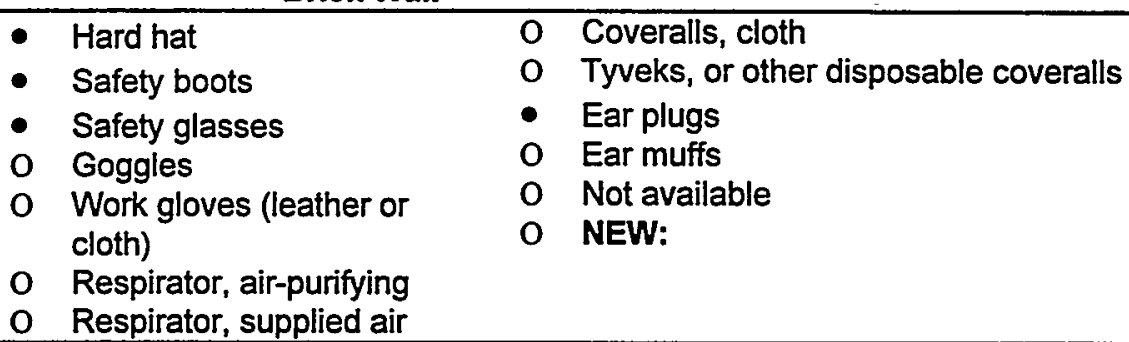 } \\
\hline $\begin{array}{l}\text { Technology Model } \\
\text { Maneuverability }\end{array}$ & \multicolumn{4}{|c|}{$\begin{array}{l}\text { Robot is remotely operated by joysticks. This allows the robot to move in any } \\
\text { direction. }\end{array}$} \\
\hline $\begin{array}{l}\text { Area of Surface } \\
\text { Decontaminated }\left(\mathrm{ft}^{2}\right)\end{array}$ & \multicolumn{4}{|l|}{130.33} \\
\hline Production Rate (ft'/h) & \multicolumn{4}{|l|}{141.66} \\
\hline $\begin{array}{l}\text { Depth of Surface } \\
\text { Removed and Method for } \\
\text { Determining Depth }\end{array}$ & \multicolumn{4}{|c|}{$\begin{array}{lll}\text { O } & \text { Surface cleaning } & \text { NEW: } \\
0 & \text { Less than coating/rust } & \text { Method: } \\
\text { - Coating/rust removal } & \\
0 & \text { Less than } 1 / 4 \text { in. } & \text { Exact quantity: } \\
0 & \text { Greater than } 1 / 4 \text { but less than } 1 / 2 \text { in. } & \text { Exact quantity:_(in.) } \\
0 & \text { Greater than } 1 / 2 \text { in. but less than } 1 \text { in. } & \text { Exact quantity:_ (in.) } \\
0 & \text { Greater than } 1 \text { in. (cracks) } & \text { Exact quantity: }\end{array}$} \\
\hline Removal Gap (in.) & \multicolumn{4}{|l|}{14.96} \\
\hline $\begin{array}{l}\text { Removal Media Usage } \\
\text { Rate (Unittft') }\end{array}$ & \multicolumn{4}{|l|}{$\begin{array}{l}\text { Type: Water } \\
\text { Quantity: } 4.31 \\
\text { Unit: gpm } \\
\end{array}$} \\
\hline \multicolumn{5}{|l|}{$\begin{array}{l}\text { Technology Model } \\
\text { Decontamination Method }\end{array}$} \\
\hline Demobilization Time (h) & \multicolumn{4}{|l|}{0.73} \\
\hline $\begin{array}{l}\text { Ending Surface } \\
\text { Condition } \\
\text { (Choose ONE of the following } \\
\text { options) }\end{array}$ & $\begin{array}{ll}\text { Metal (Steel): } \\
\text { o } & \text { Brush-off finish } \\
\text { o } & \text { Commercial } \\
& \text { finish } \\
0 & \text { Near white } \\
& \text { metal finish } \\
0 & \text { White metal } \\
& \text { finish } \\
\text { o NEW: } & \\
& \\
\text { New Category: } \\
\text { New Field: }\end{array}$ & $\begin{array}{ll} & \text { Meta } \\
0 & c \\
& 5 \\
& 1 \\
0 & 5 \\
& f \\
0 & 5 \\
0 & f\end{array}$ & $\begin{array}{l}\text { al (Other): } \\
\text { Coating/rust not } \\
\text { successfully } \\
\text { removed } \\
\text { Dull bare metal } \\
\text { finish } \\
\text { Shiny bare metal } \\
\text { finish } \\
\text { NEW: }\end{array}$ & $\begin{array}{l}\text { Masonry and Other: } \\
\text { O Coating not successfully } \\
\text { removed } \\
\text { - Coating removed } \\
\text { o Rough, bare, uneven } \\
\text { surface } \\
\text { o Smooth, bare, even } \\
\text { surface } \\
\text { O NEW: }\end{array}$ \\
\hline $\begin{array}{l}\text { Waste Volume }\left(\mathrm{ft}^{3} / \mathrm{ft}^{2}\right) \\
\text { (include only substrate removed } \\
\text { and spent media) }\end{array}$ & \multicolumn{4}{|l|}{0.246} \\
\hline $\begin{array}{l}\text { Waste Characteristics } \\
\text { (include only substrate removed } \\
\text { and spent media) }\end{array}$ & \multicolumn{4}{|c|}{ Water with suspended paint particles. } \\
\hline
\end{tabular}




\section{DECONTAMINATION TECHNOLOGIES FOR BUILDING MATERIALS}

(Continued)

\begin{tabular}{|c|c|c|c|c|c|}
\hline \multicolumn{6}{|c|}{$\begin{array}{l}\text { DEMONSTRATION INFORMATION } \\
\text { Uncoated Concrete Wall }\end{array}$} \\
\hline $\begin{array}{l}\text { Demonstration Site } \\
\text { (include city \& state) }\end{array}$ & \multicolumn{5}{|c|}{$\begin{array}{l}\text { Florida International University - Hemispheric Center for Environmental } \\
\text { Technology, Miami, FL }\end{array}$} \\
\hline Demonstration Date & \multicolumn{5}{|c|}{$\begin{array}{l}\text { Start: } 12 / 08 / 98 \\
\text { End: } 12 / 10 / 98\end{array}$} \\
\hline $\begin{array}{l}\text { Testing Organization } \\
\text { Information }\end{array}$ & \multicolumn{5}{|c|}{$\begin{array}{l}\text { Testing Organization: FIU-HCET } \\
\text { Contact Name: Cindy Zhang } \\
\text { Phone Number: (305) 348-6340 } \\
\text { e-mail: zhangc@eng.fiu.edu }\end{array}$} \\
\hline Vendor Name & \multicolumn{5}{|c|}{ Bartlett Services inc. \& Bartlett Nuclear Inc. } \\
\hline Test Engineer Name & \multicolumn{5}{|c|}{ Cindy Zhang } \\
\hline $\begin{array}{l}\text { Vendor Principal } \\
\text { Investigator Name }\end{array}$ & \multicolumn{5}{|c|}{ Sam Maggio } \\
\hline $\begin{array}{l}\text { Size of Area Assigned } \\
\text { (include units) }\end{array}$ & \multicolumn{5}{|c|}{$387.60 \mathrm{ft}^{2}$} \\
\hline $\begin{array}{l}\text { Demonstrated Surface } \\
\text { Media } \\
\text { (Choose ONE of the } \\
\text { following options) }\end{array}$ & \multicolumn{4}{|c|}{$\begin{array}{ll}\text { Metals: } \\
\text { O } & \text { Aluminum } \\
\text { O } & \text { Carbon steel } \\
\text { O } & \text { Copper } \\
\text { O } & \text { Lead } \\
\text { O } & \text { Nickel } \\
\text { O } & \text { Stainless steel } \\
\text { O } & \text { NEW: } \\
\end{array}$} & $\begin{array}{ll}\text { Other: } \\
\text { O } & \text { Ceramic } \\
\text { O } & \text { Composite } \\
\text { O } & \text { Wood } \\
\text { O } & \text { NEW: }\end{array}$ \\
\hline $\begin{array}{l}\text { Surface Media } \\
\text { Description }\end{array}$ & \multicolumn{5}{|c|}{$\begin{array}{l}\text { The uncoated concrete floor has the dimensions of } 236.5^{n} \times 236^{n} \text { with a } \\
\text { compression strength between } 4,420 \text { to } 5,660 \text { psi. }\end{array}$} \\
\hline $\begin{array}{l}\text { Demonstrated } \\
\text { Geometry } \\
\text { (Choose ONE of the } \\
\text { following options) }\end{array}$ & \multicolumn{5}{|c|}{\begin{tabular}{|llll} 
O & Equipment & O & Ceiling \\
0 & Glove box & $\bullet$ & Floor \\
O & Pipe & O & Pier \\
O & Plate & O & Wall \\
O & Structural & O & NEW: \\
0 & Tank & & \\
0 & Wire & & \\
\end{tabular}} \\
\hline \multicolumn{6}{|c|}{ The following sections are to be completed during or immediately after the demonstration: } \\
\hline Mobilization Time (h) & \multicolumn{5}{|c|}{4.33} \\
\hline $\begin{array}{l}\text { Portability Option } \\
\text { Chosen } \\
\text { (Choose ONE of the } \\
\text { following options) }\end{array}$ & \multicolumn{5}{|c|}{\begin{tabular}{|ll}
$\mathrm{O}$ & 1 person needed \\
0 & 2 people needed \\
- & forklift needed \\
$\mathrm{O}$ & trailer mounted \\
$\mathrm{O}$ & Not available \\
$\mathrm{O}$ & NEW: \\
\end{tabular}} \\
\hline $\begin{array}{l}\text { Required PPE for } \\
\text { Demonstration } \\
\\
\text { (Select all that apply - most } \\
\text { restrictive used at } \\
\text { demonstration) }\end{array}$ & \multicolumn{3}{|c|}{$\begin{array}{ll}- & \text { Hard hat } \\
\text { - Safety boots } \\
\text { - Safety glasses } \\
\text { O } & \text { Goggles } \\
\text { O Work gloves (leather or cloth) } \\
\text { O } & \text { Respirator, air-purifying } \\
\text { O } & \text { Respirator, supplied air } \\
\end{array}$} & \multicolumn{2}{|c|}{$\begin{array}{l}\text { Coveralls, cloth } \\
\text { Tyveks, or other disposable coveralls } \\
\text { Ear plugs } \\
\text { Ear muffs } \\
\text { Not available } \\
\text { NEW: }\end{array}$} \\
\hline
\end{tabular}




\section{DECONTAMINATION TECHNOLOGIES FOR BUILDING MATERIALS}

(Continued)

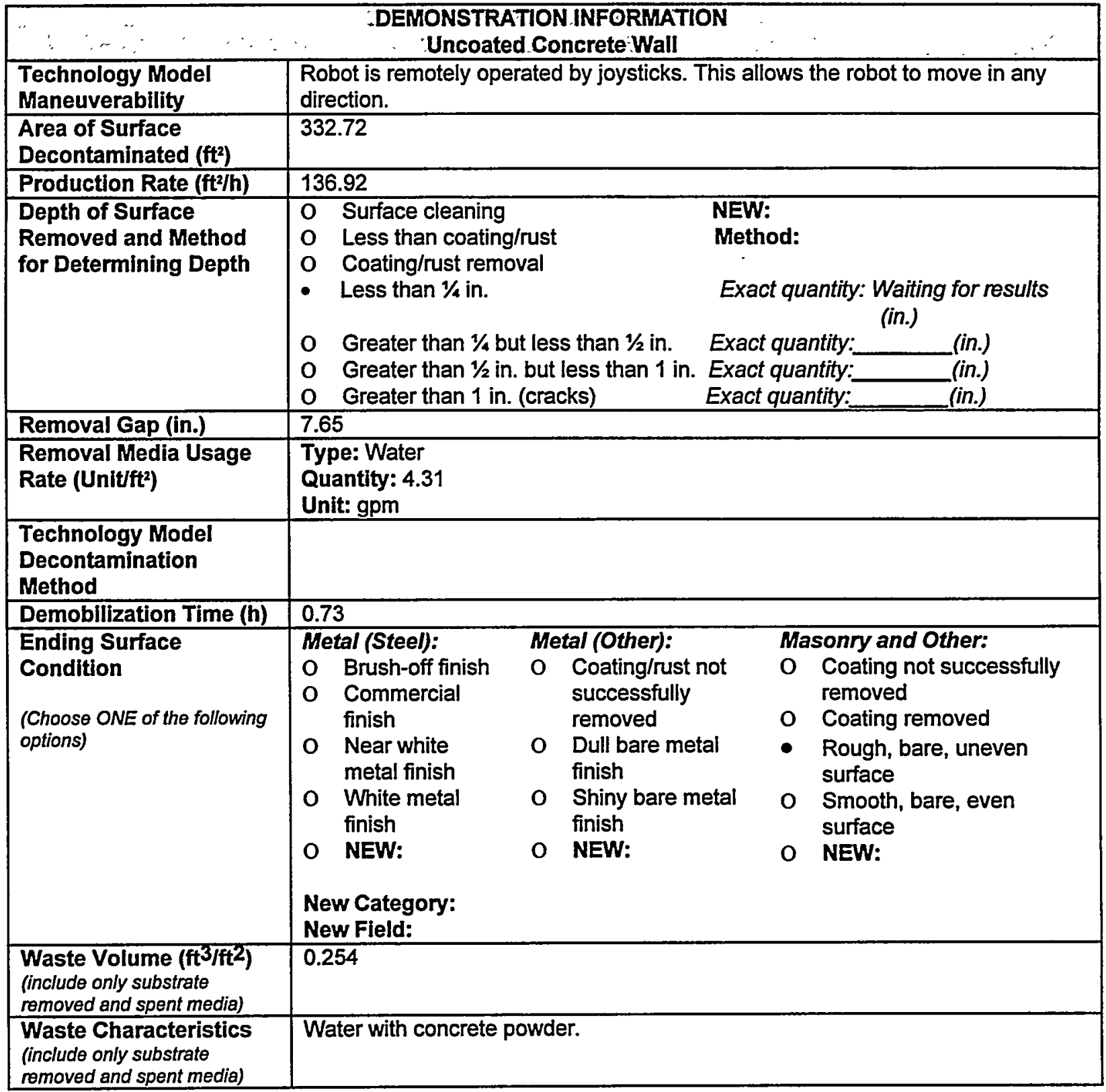




\section{DECONTAMINATION TECHNOLOGIES FOR BUILDING MATERIALS (Continued)}

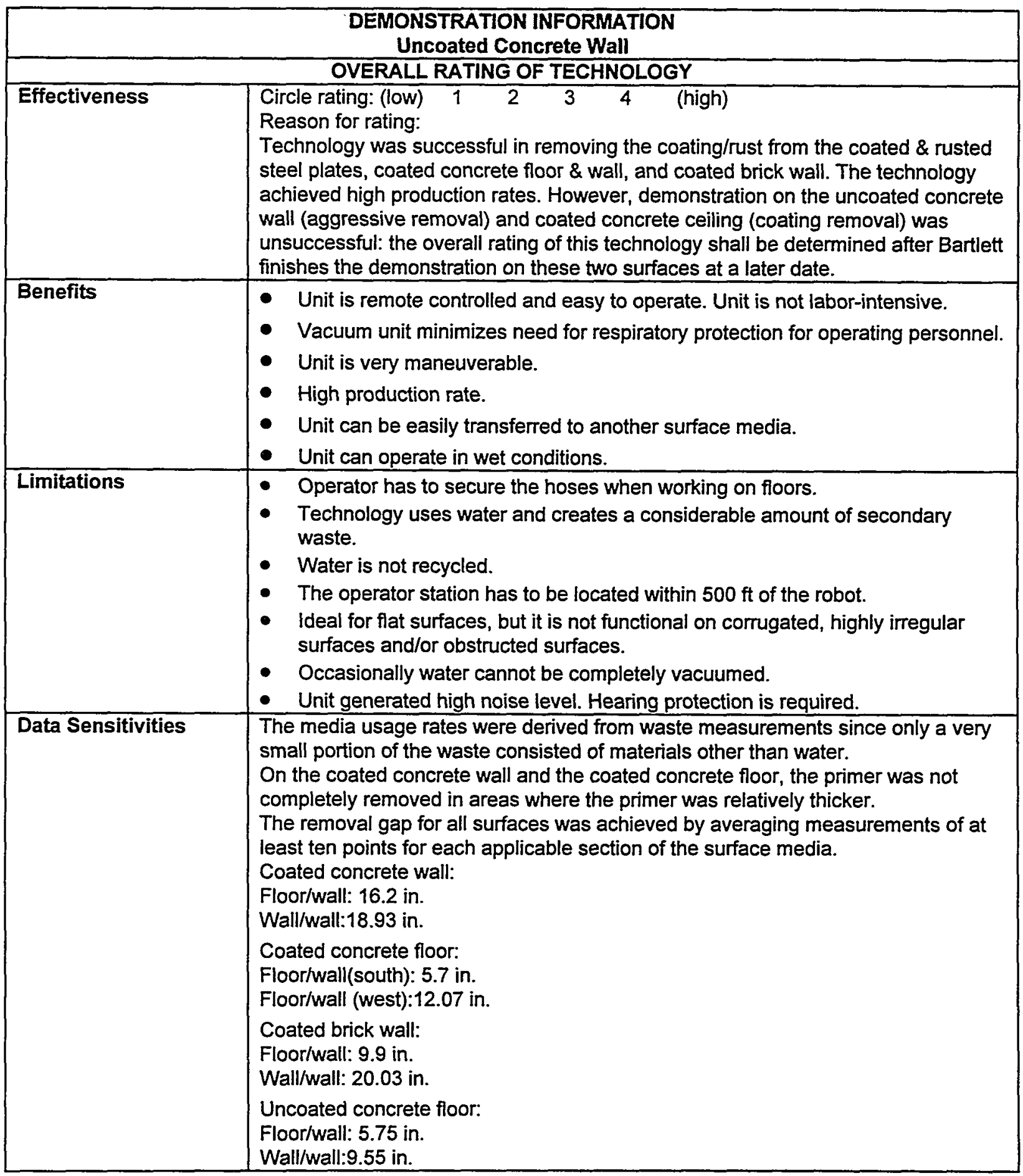




\section{DECONTAMINATION TECHNOLOGIES FOR BUILDING MATERIALS}

(Continued)

\begin{tabular}{|c|c|}
\hline$\therefore$ & $\begin{array}{l}\text { DEMONSTRATION INFORMATION } \\
\text { Uncoated Concrete Wall }\end{array}$ \\
\hline Multimedia & 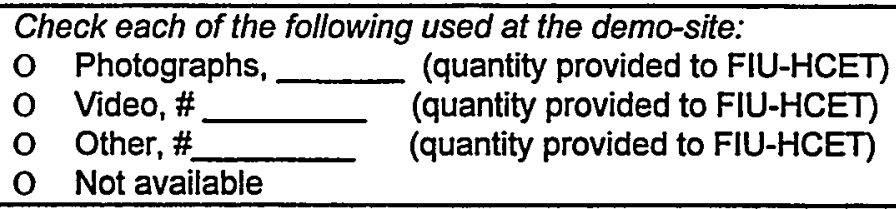 \\
\hline 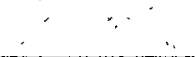 & HEALTH AND SAFETYINFORMATION \\
\hline & HEALTH AND SAFETY RATINGS \\
\hline See IUOE F & sued at the end of $F Y 99$ ) \\
\hline
\end{tabular}




\title{
Integrated Vertical and Overhead Decontamination System
}

\author{
Project Number: HCET-1998-D023
}

\section{Project objectives}

The overall objective of this subtask is to fabricate and test an innovative technology for the purpose of characterizing and decontaminating vertical and overhead structures and to transfer this technology to industry for use in reducing the cost to perform decontamination operations. The subobjectives required to meet the overall objective include the following:

- Design and fabricate a characterization system for overhead and vertical applications.

- Design and fabricate a decontamination system for overhead and vertical applications.

- Integrate and assess the system for commercial application.

- Transfer the system to industry for use throughout the DOE complex.

\section{Major milestones}

\begin{tabular}{|c|l|l|l|}
\hline Milestone No. & \multicolumn{1}{|c|}{ Milestone Description } & \multicolumn{1}{|c|}{ Completion Criteria } & \multicolumn{1}{|c|}{ Status } \\
\hline D023-M1 & Selection of Industrial Partner & $\begin{array}{l}\text { Selection of a responsible and } \\
\text { qualified vendor }\end{array}$ & $\begin{array}{l}\text { In progress } \\
\text { Scheduled completion } \\
2 / 1 / 99 .\end{array}$ \\
\hline D023-M2 & $\begin{array}{l}\text { Approved Design } \\
\text { Specifications for the } \\
\text { Decontamination System }\end{array}$ & $\begin{array}{l}\text { Approval of final design } \\
\text { specifications for the } \\
\text { decontamination system }\end{array}$ & $\begin{array}{l}\text { Scheduled completion } \\
4 / 6 / 99 .\end{array}$ \\
\hline D023-M3 & $\begin{array}{l}\text { Fabrication of Decontamination } \\
\text { System }\end{array}$ & $\begin{array}{l}\text { Complete fabrication of } \\
\text { decontamination system unit }\end{array}$ & $\begin{array}{l}\text { Scheduled completion } \\
7 / 30 / 99\end{array}$ \\
\hline D023-M4 & $\begin{array}{l}\text { Field Testing of } \\
\text { Decontamination System }\end{array}$ & $\begin{array}{l}\text { Completion of testing of } \\
\text { decontamination unit at FIU- } \\
\text { HCET Test Site }\end{array}$ & $\begin{array}{l}\text { Scheduled completion } \\
8 / 16 / 99 .\end{array}$ \\
\hline D023-M5 & $\begin{array}{l}\text { Design Drawings for the } \\
\text { Characterization System }\end{array}$ & $\begin{array}{l}\text { Approval of final design } \\
\text { specifications for the } \\
\text { characterization system }\end{array}$ & $\begin{array}{l}\text { Scheduled completion } \\
6 / 1 / 99\end{array}$ \\
\hline D023-M6 & $\begin{array}{l}\text { Fabrication of Characterization } \\
\text { System }\end{array}$ & $\begin{array}{l}\text { Complete fabrication of } \\
\text { characterization system }\end{array}$ & $\begin{array}{l}\text { Scheduled completion } \\
9 / 30 / 99 .\end{array}$ \\
\hline D023-M7 & $\begin{array}{l}\text { Testing the Characterization } \\
\text { System }\end{array}$ & $\begin{array}{l}\text { Completion of } \\
\text { characterization system testing } \\
\text { at FIU-HCET }\end{array}$ & $\begin{array}{l}\text { Scheduled completion } \\
10 / 15 / 99\end{array}$ \\
\hline D023-M8 & $\begin{array}{l}\text { Final report on the } \\
\text { Decontamination and } \\
\text { Characterization System. }\end{array}$ & $\begin{array}{l}\text { Deliver final report to DDFA- } \\
\text { determined distribution list }\end{array}$ & $\begin{array}{l}\text { Scheduled completion } \\
11 / 30 / 99\end{array}$ \\
\hline
\end{tabular}

\section{Significant events}

- A Scope of Work document has been generated. This document details the requirements of the integrated decontamination, deployment mechanism, and waste collection system. This document is intended to help vendors in the development of their proposals to Florida International University's Hemispheric Center for Environmental Technology (FIU-HCET). 
Several conceptual configuration AutoCad figures are provided in this document. This Scope of Work is presented in Appendix A. After internal review this document will be provided to a list of already identified technology vendors capable of developing responsive and competitive proposals.

- The performance specifications were generated describing the project scope, system requirements, project deliverables, and requirements for completion of Scope of Work. Refer to the FY98 year-end report and the FY99 Project Technical Plan for "Integrated Vertical and Overhead Decontamination System."

\section{Accomplishments and technical progress}

- During FY98 an extensive search for decontamination technologies was conducted. Several sources were utilized, including Remedial Action Program Information Center (RAPIC), FIUHCET databases for decontamination technologies, and others. Eight technologies were shortlisted for comprehensive analysis to determine the optimum technology to deploy. The criteria for final selection were

- Removal capabilities

- Production rates

- Cost information

- Waste generation

- Health and safety.

- Based on the comprehensive technology analysis, the Marcrist Diamond Wheel Shaving Technology was selected for deployment.

- In addition to the selection of the optimum decontamination technology, the characterization technology and deployment platform selection criteria were determined, and screening of potential technologies to integrate with the selected decontamination technology was commenced.

- For additional details on accomplishments and technical progress, refer to the Integrated Vertical and Overhead Decontamination System FY98 Year-End Report.

\section{Assessment of current status}

A performance document and initial conceptual drawing have been developed for the decontamination system. A decontamination technology has been selected, and a deployment mechanism has been identified. A commercial partner needs to be selected and contracted to complete the design and manufacturing of the decontamination system. To this end, a Scope of Work document (see Appendix A) has been developed and will be distributed among a list of technically capable vendors. 


\section{Plans for the next two months}

Activities for the next two months include the following:

- Transmit the Scope of Work document to selected vendors and select vendor based on qualifications and response to Scope of Work document.

- Complete Test Plan for the development of a test site for the characterization system.

- Receive preliminary Title I Design documentation from selected vendor.

\section{FIU-HCET collaborators}

Leonel E. Lagos, (305) 348-1810

Man Young Cheung, (305) 348-6653

Richard Musgrove, (305) 348-6622 


\section{Appendix A. Scope of Work for \\ Development of an Integrated Vertical and Overhead Decontamination System}

\subsection{Problem Definition}

The treatment of radioactively contaminated concrete and metal surfaces is a concern during the deactivation and decommissioning (D\&D) process. As buildings undergo D\&D, concrete, brick, and metal surfaces contaminated with radionuclides, such as uranium and technetium-99, and others must be decontaminated before final disposal. It is estimated that 23 million cubic meters of concrete and 600,000 tons of metal will require disposition as 1200 buildings undergo the D\&D process.

Florida International University's Hemispheric Center for Environmental Technology (FIU-HCET), through its Technology Assessment Program, has been able to identify the necessary requirements and tools for addressing some of these needs.

To perform D\&D activities on these surfaces in a cost-effective and safe manner, the situation requires the following:

- The development of an integrated decontamination and robotic deployment system for removing layers of concrete from wall, ceiling, and floor surfaces as well as removal of coatings from metal surfaces combined with a real-time characterization information system. Such a system has not been developed yet, although FIU-HCET believes that technologies in the characterization and decontamination areas already exist.

- A decontamination system that can be remotely operated from a safe distance, thus providing a safer working environment for workers and alleviating workers' Health and Safety (H\&S) concerns.

- A robotic-operated decontamination system to reduce workload on workers in large areas requiring $D \& D$. Current manual labor methods employed to perform the decontamination of concrete wall, ceiling, floor, and metal surfaces are labor-intensive as opposed to robotic operation. The treatment of large areas with a robotic deployment/decontamination system will reduce labor as well as cost and schedule.

- A system that generates small amounts of secondary waste. Current decontamination systems generate large volumes of secondary waste (i.e., high-pressure water systems).

- The integration of a nuclear-rated close loop waste collection system equipped with nuclear-rated high-efficiency particulate air (HEPA) filtering system.

- The transfer of the complete integrated system to industry for deployment throughout the DOE complex.

\subsection{Project Scope}

To design a decontamination system (a remote-controlled, free-climbing robot) using a shrouded concrete shaving decontamination unit. The decontamination system shall employ a self-propelled remote joystick-operated robotic deployment system. Adhesion to the surface shall be achieved by 
using negative pressure, enabling the robot to perform on vertical, horizontal, and overhead surfaces. This system shall also be equipped with a waste collection and handling system rated for nuclear use. This waste system shall be a closed loop system able to capture the waste scabbled from the surface. The captured waste shall be sent to a waste collection system equipped with an ultra-high performance HEPA vacuum system. A controlled seal drum fill system shall allow the operator to fill, seal, remove, and replace the waste drum under controlled vacuum conditions, thus minimizing the risk of releasing airborne contamination and exposing the workers to possible contamination.

The decontamination, robotic deployment, and waste collection systems shall be integrated to perform decontamination activities on vertical, horizontal, and overhead concrete, brick, and metal surfaces.

\subsection{System Requirements}

The following list contains the requirements for the integrated deployment/decontamination system. Any deviations from the list must be fully detailed by the technology vendor in the proposal to FIUHCET.

- System must be capable of removing $1 / 8$ to $1 / 4$ inch of concrete per pass.

- System must be capable of a removal path of greater than 9 inches wide per pass on concrete or metal surfaces.

- System must be capable of removing coating from metal and concrete surfaces.

- System should have a depth of removal adjusting mechanism to lower the concrete shaving decontamination unit on to the surface after it is turned on.

- System must be capable of working in horizontal, vertical, and overhead deployment positions.

- Vertical reach of the system shall be at least $20 \mathrm{ft}$ and no more than $40 \mathrm{ft}$.

- Removal gaps near corners or edges of walls or ceilings shall not exceed 10 inches. Minimizing the removal gap on any side of the robot is desirable.

- Vendor shall incorporate a decontamination technology capable of achieving the above requirements. It is highly desirable that the vendor incorporates a diamond wheel (drum) system as the base for the decontamination technology. Vendor can consider using other suitable decontamination technology as well.

- System must capture all decontamination media and shall not generate hazardous loose waste.

- All waste must be dry with waste collection into 38- or 55-gallon drums.

- The system must have nuclear-grade bag-in bag-out HEPA filtration as the final stage of filtration.

- System must be completely field-mobile.

- All electrical work must meet Occupational Safety and Health Administration (OSHA) standards and must be watertight.

- Robot wheels shall be electric or pneumatic motor-driven with speed control. 
- Safety features should stop the decontamination system when loss of contact with the surface is detected.

- Handle bars or cage structure on the topside of the robot should enable easy handling and protection from accidental falls upside down.

- Safety tether system should support the free-climbing robot in accidental falls.

- The robot shall be as light as possible.

Conceptual design drawings have been provided. Figures 1 through 4 show the conceptual layout of the decontamination system and integrated system. These conceptual designs are intended to show the functions of the system and are not necessarily the final design. These figures are intended to guide the prospective bidder in the types of designs and concepts that best address the needs stated above.

\subsection{Alternative Seal for the Decontamination System}

Inflatable seals could provide a substitute seal solution instead of an air pillow. This product can be procured from companies such as Seal Master Corporation (http://sealmaster.com/). The inflatable seals would decrease the size and removal gap of the decontamination system.

\subsection{Alternative Decontamination System}

The development of the decontamination system requires the integration of an aggressive decontamination system able to scabble concrete surfaces from $1 / 8$ to $1 / 4$ inch per pass. Based on FIU-HCET experience with decontamination systems, it is recommended to bidders to integrate a diamond impregnated wheel drum as the basis for the decontamination technology. This product can be procured from companies such as Marcrist Industries from the United Kingdom (U.K.) or similar companies with the same product. Figures 1 and 2 show the conceptual designs of the decontamination system for robotic arm deployment and free-climbing robotic deployment. Other proposed technologies will also be considered as long as they meet system design requirements.

\subsection{Alternative Deployment Methods}

Alternative deployment for the decontamination system can be accepted if it meets the requirements as specified above. An alternative design can be a robotic arm holding the decontamination system. The robotic arm deployment and the free climbing robotic deployment configurations are shown in Figures 3 and 4 . Other deployment alternatives may also be considered as long as the system requirements are met.

\subsection{Project Phases}

The procurement of the entire integrated system (decontamination, deployment mechanism, and waste collection systems) shall include the use of five main phases. At the completion of each phase, the approval of the FIU-HCET D\&D Program Manager is required. The execution of one task is contingent on the completion and approval of the previous task. Funding is not authorized to begin the next task unless it is approved by FIU-HCET. 
1. Title I Design - Provide initial design of system and review estimated costs for completion. FIUHCET will review and approve $20 \%$ completion of design drawings. FIU-HCET will also approve the expenditure of long lead-time procurement items. Upon the approval and completion of Title I, FIU-HCET will pay $20 \%$ of contract amount.

2. Title II Design - FIU-HCET will review and approve the final design of the Decontamination/ Deployment System and review estimated costs for completion. Upon approval and completion of Title II design, an additional $30 \%$ of the contract amount will be paid.

3. Title III Design - FIU-HCET will monitor the fabrication of the system to ensure the specifications and design drawings are being followed. At the completion of the fabrication FIUHCET will authorize an additional $30 \%$ of the contract amount to be paid.

4. Operations - FIU-HCET will witness the system in action under different conditions such as horizontal/vertical deployment, concrete/metal surface. This phase shall be conducted at FIUHCET testing facilities or a Department of Energy (DOE) facility identified and approved by FIU-HCET and the Federal Energy Technology Center (FETC). The vendor using a format approved by FIU-HCET will develop a detailed operations and maintenance manual. This manual shall be presented to FIU-HCET for final review and approval.

5. Closeout - Upon successful field testing of the system, FIU-HCET personnel will be trained on the operation and maintenance of the system. Upon successful completion, the final $20 \%$ of the contract money will be paid.

\subsection{Conceptual Design of the Integrated System}

The following figures represent conceptual design of the potential deployment, decontamination technologies, and waste collection system. These figures are only presented here to aid prospective bidders in the development of the integrated system. Vendors are encouraged to present other ideas and designs meeting the specification listed above. 


\subsection{Conceptual Design of the Decontamination System for Robotic Arm Deployment}

Front View

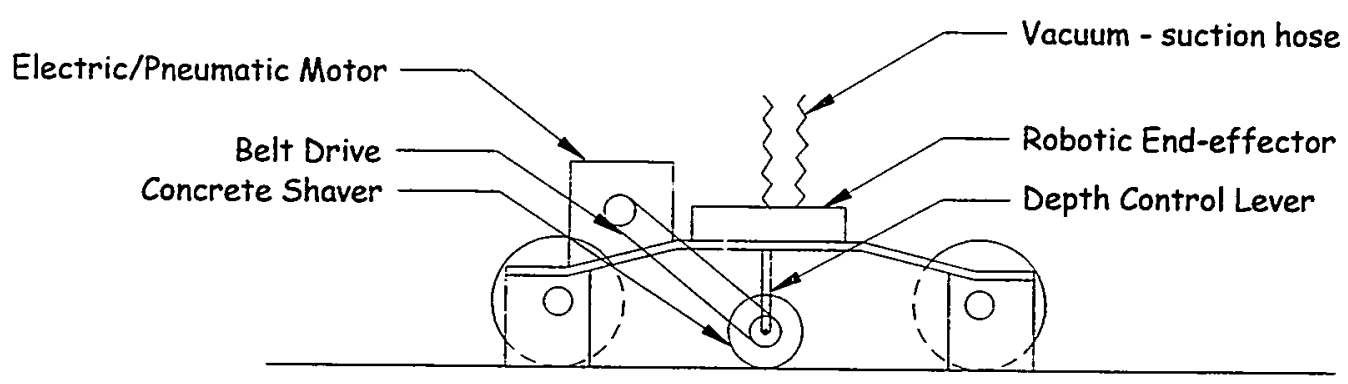

Top View

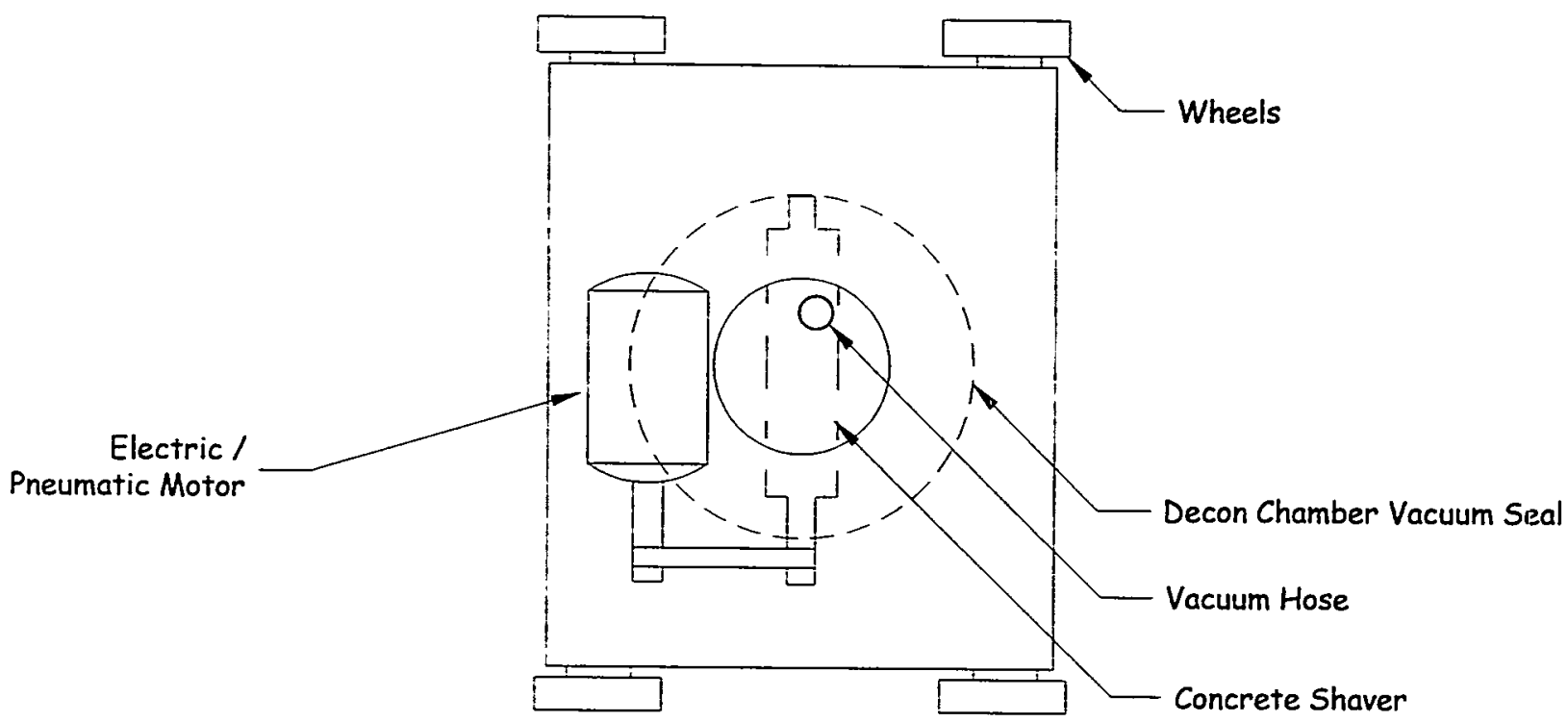

Figure 1. Conceptual Design of the Decontamination System for Robotic Arm Deployment. 


\subsection{Conceptual Design of the Decontamination System for Free Climbing Robotic Deployment:}

\section{Front View}

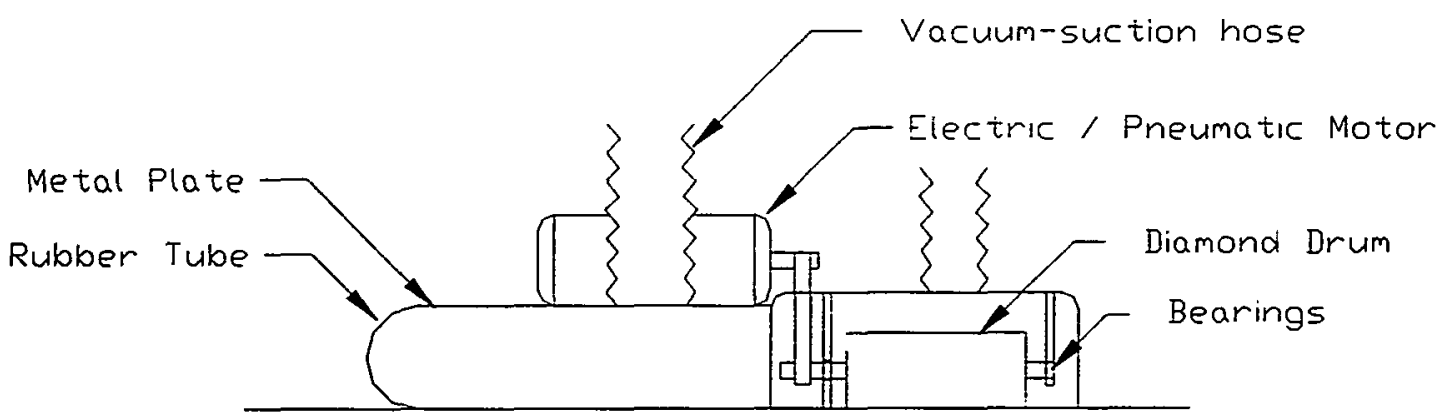

Top View

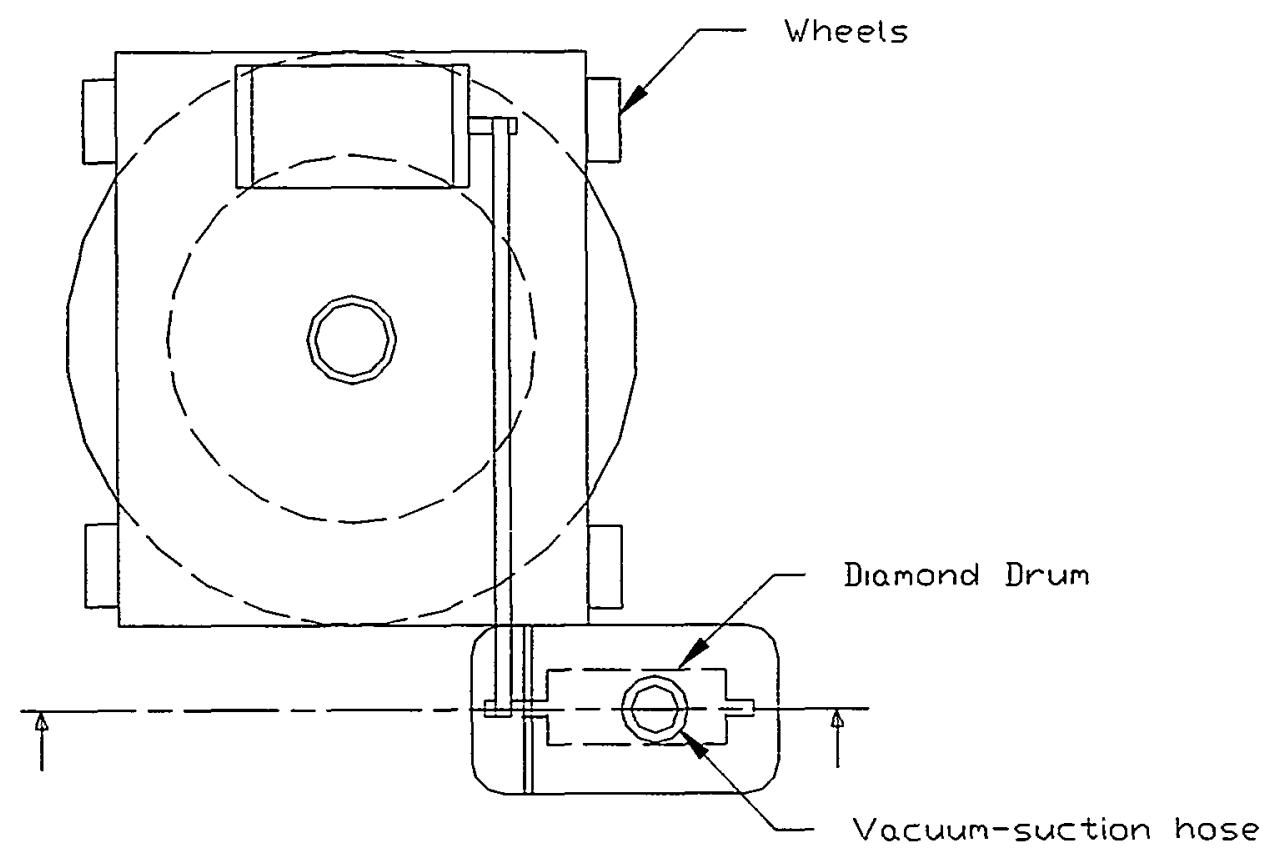

Figure 2. Conceptual Design of the Free-Climbing Robot Decontamination System for Vertical and Overhead Deployment. 


\subsection{Conceptual Design of the Integrated System for Robotic Arm Deployment}

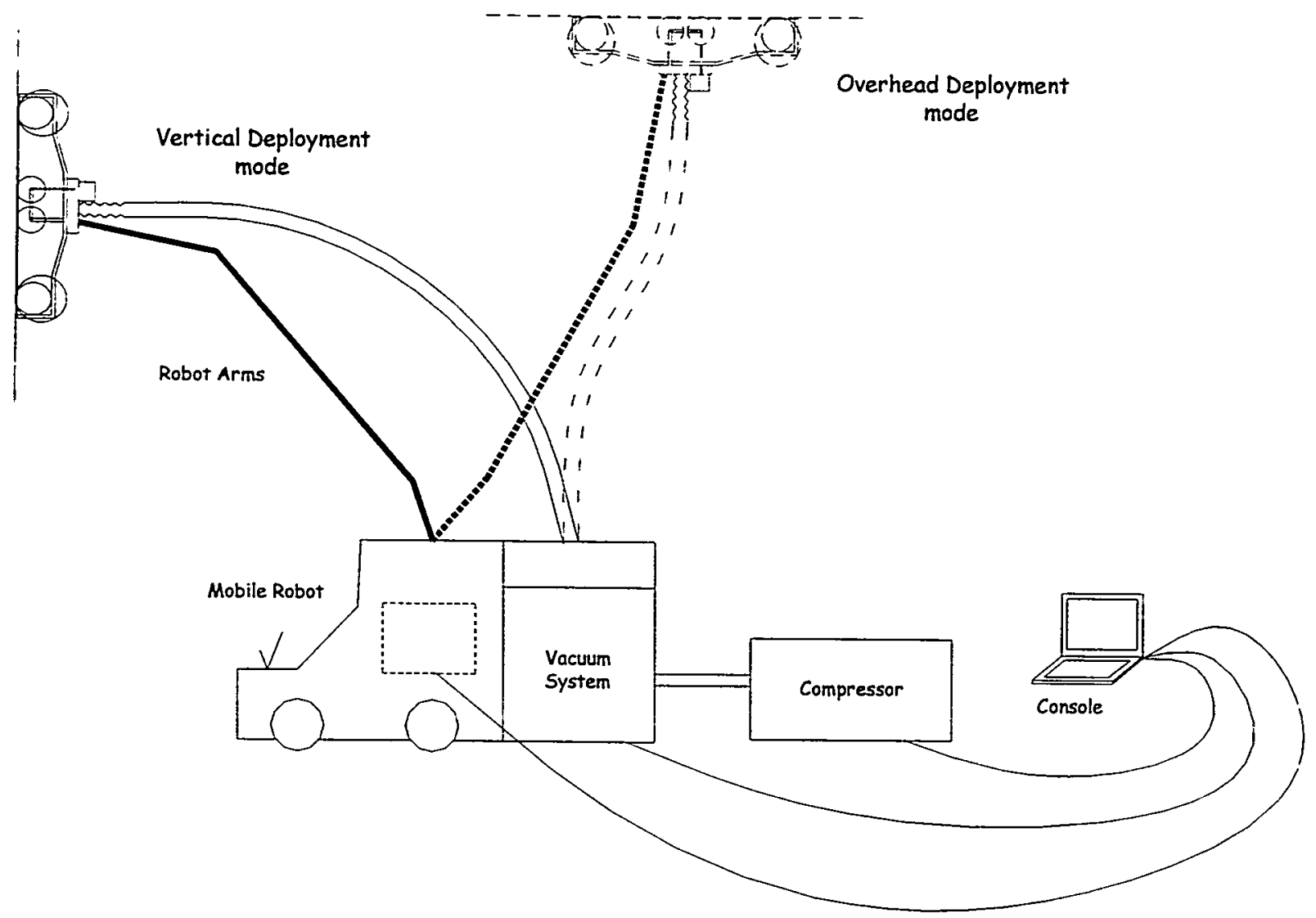

Figure 3. Robotic Deployment Conceptual Design for Robotic Arm and Free-Climbing Robot. 


\subsection{Conceptual Design of the Integrated System for Free Climbing Rohotic Deployment:}

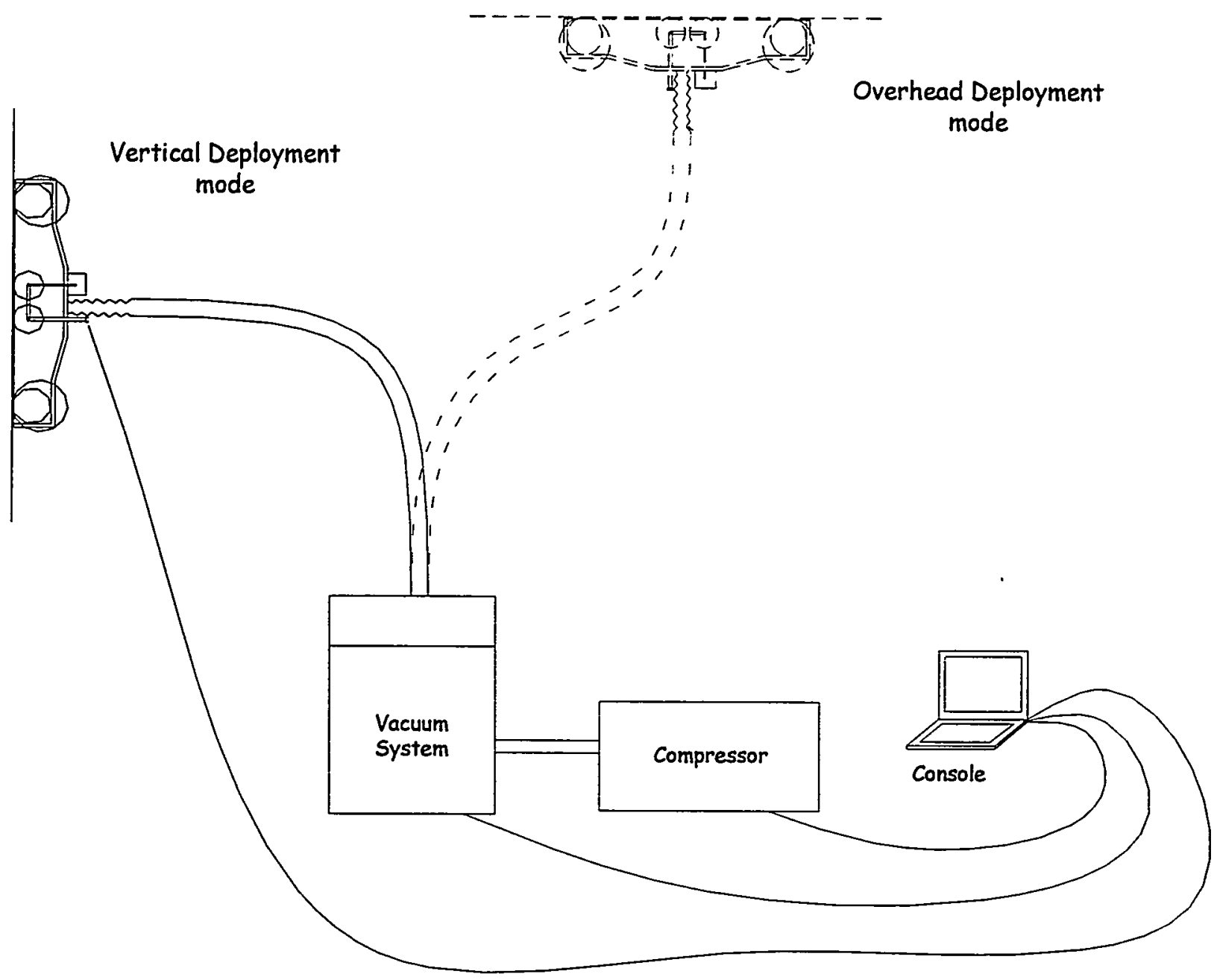

Figure 4. Integrated Vertical and Overhead Decontamination System-Conceptual Design. 


\title{
Large-Scale Demonstration and Deployment Project- Technology Information System (LSDDP-TIS)
}

\author{
Project Number: HCET-1998-D039
}

\section{Project objectives}

Within the DOE complex, there are some 10,000 buildings that require deactivation and decommissioning (D\&D). These facilities present an immense array of problems and challenges for D\&D project managers who must investigate and screen scores of candidate technologies to select the most appropriate one(s) for their specific remediation problems. The search for candidate technologies can be arduous and involve several sources of varying reliability. The Large-Scale Technology Demonstration and Deployment Project Technology Information System (LSDDP-TIS) will facilitate the search and selection process by providing D\&D managers with ready access to an extensive information base of DOE-screened environmental technologies.

The objectives of this subtask include the following:

- Collect technology information from LSDDP technology screenings and other reliable sources

- Compile a searchable database to serve as an aid to decision-makers for identifying candidate technologies for future LSDDPs or for addressing specific problems.

\section{Major milestones}

\begin{tabular}{|c|c|c|c|}
\hline Milestone No. & Milestone Description & Completion Criteria & Status \\
\hline D039-M1 & Release 1 of the TIS & $\begin{array}{l}\text { User Access to the functional } \\
\text { LSDDP-TIS via the Internet }\end{array}$ & $\begin{array}{l}\text { Completed on } \\
\text { schedule } 1 / 4 / 99 \text {. }\end{array}$ \\
\hline D039-M2 & $\begin{array}{l}\text { Information from new } \\
\text { LSDDPs incorporated }\end{array}$ & $\begin{array}{l}\text { Screening data from new } \\
\text { LSDDPs accessible through TIS }\end{array}$ & $\begin{array}{l}\text { Ongoing. Scheduled } \\
\text { for completion } 3 / 1 / 99\end{array}$ \\
\hline D039-M3 & $\begin{array}{l}\text { Information on DOE's } \\
\text { baseline technologies and } \\
\text { FIU-HCET's Technology } \\
\text { Assessment Program } \\
\text { incorporated }\end{array}$ & $\begin{array}{l}\text { Information on DOE baseline and } \\
\text { FIU-HCET-assessed technologies } \\
\text { accessible through TIS }\end{array}$ & $\begin{array}{l}\text { Ongoing. Scheduled } \\
\text { for completion } 3 / 1 / 99\end{array}$ \\
\hline D039-M4 & $\begin{array}{l}\text { Information from DOE } \\
\text { databases incorporated }\end{array}$ & $\begin{array}{l}\text { Data from DOE designated } \\
\text { databases accessible through TIS }\end{array}$ & $\begin{array}{l}\text { Ongoing. Scheduled } \\
\text { for completion } 4 / 30 / 99\end{array}$ \\
\hline D039-M5 & $\begin{array}{l}\text { TIS linked to other D\&D } \\
\text { Technology Web Sites }\end{array}$ & $\begin{array}{l}\text { Users of TIS provided with } \\
\text { hyperlinks to other technology } \\
\text { web sites }\end{array}$ & $\begin{array}{l}\text { Not scheduled to begin } \\
\text { until May } 1999\end{array}$ \\
\hline$\overline{D 039-M 6}$ & Final Report & $\begin{array}{l}\text { Final report on results of the } \\
\text { project delivered to DOE. }\end{array}$ & $\begin{array}{l}\text { Scheduled for } \\
\text { completion } 10 / 31 / 99\end{array}$ \\
\hline D039-M7 & $\begin{array}{l}\text { DDFA decision on other } \\
\text { media to access TIS }\end{array}$ & $\begin{array}{l}\text { Users able to access TIS } \\
\text { information via other media such } \\
\text { as telephone and return fax. }\end{array}$ & $\begin{array}{l}\text { If approved, scheduled } \\
\text { for FY- } 00 \text {. }\end{array}$ \\
\hline
\end{tabular}




\section{Significant events}

The first release of the fully functional TIS debuted on January 4, 1999.

\section{Accomplishments and technical progress}

- The results of 131 CP-5 LSDDP technology screenings have been verified by Florida International University's Hemispheric Center for Environmental Technology (FIU-HCET) and entered into the TIS database. Missing data sheets for 14 technologies were tracked down by FIU-HCET and have been received and entered into the database.

- Of 183 technologies screened at the Fernald Environmental Management Project (FEMP) LSDDP, 8 data sheets are outstanding; 170 of the 175 received have been verified by FIU-HCET and entered into the TIS database. The remaining 5 data sheets have incomplete data. On January 12,1999 , a report listing the technologies that have incomplete data was sent to Mr. Mark Peters, Project Manager at Fernald, requesting the additional information.

- Data sheets on all 41 technologies that were screened in connection with the Hanford C-Reactor LSDDP have been received and are currently being verified for data entry.

- Recommendations by the beta testers for enhancement to the TIS are being reviewed, assessed, and, where appropriate, implemented. Suggestions on improving the functionality of the system have been provided by Mr. Dan Young, a support contractor for FETC and are being reviewed for implementation into future versions of the system.

- Data sheets from four new LSDDPs have been requested. Summarized below are the number that have been received to date. Data entry into the TIS database will commence in February.

\begin{tabular}{|l|c|c|}
\hline LSDDP Site & $\begin{array}{c}\text { \# of Technologies Screened } \\
\text { To Date at LSDDP Site }\end{array}$ & $\begin{array}{c}\text { \# of Data Sheets Received } \\
\text { To Date }\end{array}$ \\
\hline $\begin{array}{l}\text { Mound Environmental } \\
\text { Management Project (MEMP) }\end{array}$ & 62 & 62 \\
\hline Savannah River Site (SRS) & 27 & 27 \\
\hline $\begin{array}{l}\text { Idaho National Environmental } \\
\text { Engineering Laboratory } \\
\text { (INEEL) }\end{array}$ & 123 & 48 \\
\hline $\begin{array}{l}\text { Los Alamos National } \\
\text { Laboratory (LANL) }\end{array}$ & 29 & 29 \\
\hline
\end{tabular}

- Review of DOE's Baseline Technologies, FETC's Technology Database, and FIU-HCET's Technology Assessment Program (TAP) data for inclusion into the TIS has begun. Summarized below are the number of technologies that have been reviewed. Data entry will commence in February. 


\begin{tabular}{|l|c|}
\hline \multicolumn{1}{|c|}{ Databases } & \multicolumn{1}{|c|}{$\begin{array}{l}\text { \# of Technologies } \\
\text { Reviewed To Date }\end{array}$} \\
\hline $\begin{array}{l}\text { DOE's Baseline } \\
\text { Technologies }\end{array}$ & 9 \\
\hline $\begin{array}{l}\text { FETC's Technology } \\
\text { Database }\end{array}$ & 34 \\
\hline FIU-HCET's TAP & 5 \\
\hline
\end{tabular}

\section{Assessment of current status}

The system development and data acquisition phases of the project are proceeding on schedule and no major problems are foreseen.

\section{Plans for the next two months}

- Further attempts will be made to obtain missing data sheets from the FEMP Plant-1 LSDDP and the INEEL LSDDP. Once received, they will be verified and entered into the database.

- Technology information on baseline technologies listed in the DOE's Preferred Alternative Matrices (PAM) will be incorporated into the TIS database.

- Information from FETC's technology database and FIU-HCET's Technology Assessment Program will be incorporated into the TIS database.

FIU-HCET collaborator

Mabel Acosta, (305) 348-6650 


\title{
Ex-Situ Large-Bore Pipe Decontamination and Characterization System
}

\author{
Project Number: HCET-1997-D017
}

\section{Project objectives}

The deactivation and decommissioning of 10,000 buildings in the U.S. Department of Energy (DOE) complex will require the disposition of miles of pipe. In particular, the disposition of large-bore pipe presents difficulties in the areas of decontamination and characterization. This pipe is potentially contaminated internally as well as externally. This situation requires a system capable of decontaminating and characterizing both the internal and external surfaces of the pipe. Current decontamination and characterization systems are not designed for application to this geometry, necessitating, in many cases, direct disposal of the piping systems. Once disposed of, the pipe often creates voids in the disposal cell, requiring the pipe to be cut in half or filled with a grout material. These methods are labor-intensive and costly to perform on large volumes of pipe. Direct disposal does not take advantage of recycling, which would provide monetary dividends as a result of the disposition of large-bore pipe.

To facilitate the decontamination and characterization of large-bore piping and thereby reduce the volume of piping required for disposal, the following objectives have been established:

- A detailed analysis will be conducted to document the pipe remediation problem set. (completed FY97)

- Determine potential technologies to solve this remediation problem set. (completed FY97)

- Design and laboratory test potential decontamination and characterization technologies. (completed FY97)

- Fabricate a prototype system. (FY98 and FY99)

- Provide a cost-benefit analysis of the proposed system. (preliminary completed FY98)

- Deploy the system. (FY99 and beyond)

\section{Major milestones}

\begin{tabular}{|c|l|l|l|}
\hline Milestone No. & Milestone Description & \multicolumn{1}{|c|}{ Completion Criteria } & \multicolumn{1}{c|}{ Status } \\
\hline D017-M1 & $\begin{array}{l}\text { Title III of the } \\
\text { decontamination system } \\
\text { complete }\end{array}$ & $\begin{array}{l}\text { The completion of Title III provides for a } \\
\text { complete decontamination system ready for } \\
\text { a field assessment. }\end{array}$ & $\begin{array}{l}\text { Ongoing. } \\
\text { Scheduled } \\
\text { completion } 3 / 31 / 99\end{array}$ \\
\hline D017-M2 & $\begin{array}{l}\text { Field testing of the } \\
\text { decontamination system }\end{array}$ & $\begin{array}{l}\text { The decontamination system will be tested } \\
\text { to ensure the performance specifications are } \\
\text { met. This will be accomplished by } \\
\text { witnessing the cleaning of five tons of pipe } \\
\text { of various diameters. }\end{array}$ & $\begin{array}{l}\text { Ongoing. } \\
\text { Scheduled } \\
\text { completion } 4 / 20 / 99\end{array}$ \\
\hline
\end{tabular}




\begin{tabular}{|c|c|c|c|}
\hline Milestone No. & Milestone Description & Completion Criteria & Status \\
\hline D017-M3 & $\begin{array}{l}\text { Close-out of } \\
\text { decontamination system }\end{array}$ & $\begin{array}{l}\text { This milestone requires the completion of } \\
\text { all required activities, including } \\
\text { operation/maintenance procedures. Five } \\
\text { people from FIU-HCET will be trained on } \\
\text { the operation and maintenance of the } \\
\text { system. The completed system will be } \\
\text { turned over to FIU-HCET for operation and } \\
\text { integration with the characterization system. }\end{array}$ & $\begin{array}{l}\text { Ongoing. } \\
\text { Scheduled } \\
\text { completion } 5 / 26 / 99\end{array}$ \\
\hline D017-M4 & $\begin{array}{l}\text { Title I of the } \\
\text { characterization system } \\
\text { complete }\end{array}$ & $\begin{array}{l}\text { FIU-HCET will approve initial design } \\
\text { details of the characterization system and } \\
\text { the costs associated with the } \\
\text { characterization system. }\end{array}$ & $\begin{array}{l}\text { Completed } \\
11 / 30 / 98\end{array}$ \\
\hline D017-M5 & $\begin{array}{l}\text { Title II of the } \\
\text { characterization system } \\
\text { complete }\end{array}$ & $\begin{array}{l}\text { FIU-HCET will approve initial design } \\
\text { details of the characterization system and } \\
\text { the costs associated with the } \\
\text { characterization system. }\end{array}$ & $\begin{array}{l}\text { Scheduled } \\
\text { completion } \\
2 / 16 / 99^{*}\end{array}$ \\
\hline D017-M6 & $\begin{array}{l}\text { Title III of the } \\
\text { characterization system } \\
\text { complete }\end{array}$ & $\begin{array}{l}\text { The completion of Title III provides for a } \\
\text { complete characterization system ready for } \\
\text { a field assessment. }\end{array}$ & $\begin{array}{l}\text { Scheduled } \\
\text { completion } \\
7 / 28 / 99^{*}\end{array}$ \\
\hline D017-M7 & $\begin{array}{l}\text { Field testing the } \\
\text { characterization system }\end{array}$ & $\begin{array}{l}\text { The characterization system will be tested to } \\
\text { verify that it meets performance } \\
\text { requirements by characterizing five tons of } \\
\text { pipe of various sizes and contaminant types. }\end{array}$ & $\begin{array}{l}\text { Scheduled } \\
\text { completion } \\
\text { 9/14/99* }\end{array}$ \\
\hline $\mathrm{D} 017-\mathrm{M} 8$ & $\begin{array}{l}\text { Close-out of } \\
\text { characterization system }\end{array}$ & $\begin{array}{l}\text { This milestone requires the completion of } \\
\text { all required activities, including } \\
\text { operation/maintenance procedures. Five } \\
\text { people from FIU-HCET will be trained on } \\
\text { the operation and maintenance of the } \\
\text { system. The completed system will be } \\
\text { turned over to FIU-HCET for operation and } \\
\text { integration with the decontamination } \\
\text { system. }\end{array}$ & $\begin{array}{l}\text { Scheduled } \\
\text { completion } \\
11 / 30 / 99^{* *}\end{array}$ \\
\hline D017-M9 & $\begin{array}{l}\text { Final Report on the } \\
\text { decontamination and } \\
\text { characterization system }\end{array}$ & $\begin{array}{l}\text { Final report detailing the technology } \\
\text { assessment process and the design, } \\
\text { fabrication, and testing of the system will be } \\
\text { completed and issued. The final report will } \\
\text { be distributed through the Remedial Action } \\
\text { Program Information Center (RAPIC) and } \\
\text { the DDFA mailing list data base and will be } \\
\text { available on the FIU-HCET Home Page. }\end{array}$ & $\begin{array}{l}\text { Scheduled } \\
\text { completion } \\
11 / 30 / 99\end{array}$ \\
\hline D017-M10 & $\begin{array}{l}\text { Large-scale field } \\
\text { deployment of ex-situ } \\
\text { large-bore pipe } \\
\text { characterization and } \\
\text { decontamination system. }\end{array}$ & $\begin{array}{l}\text { The integrated characterization and } \\
\text { decontamination system will be deployed at } \\
\text { an environmental restoration site. }\end{array}$ & $\begin{array}{l}\text { Scheduled } \\
\text { completion } 1 / 19 / 00\end{array}$ \\
\hline
\end{tabular}

*This date has slipped 18 days due to problem with procurement of conveyor.

**This date has been moved back to allow for a more extensive demonstration to be completed at a commercial site. 


\section{Significant events}

- The blast cabinet and conveyor system for the decontamination system has been fabricated. The major system component fabrication will be complete in January 1999. The initial run-off of the system is scheduled for the second week in February 1999.

- The design drawings for the ventilation system container are complete. The container was inspected by Florida International University's Hemispheric Center for Environmental Technology (FIU-HCET) on January 20, 1999, and will be delivered to the decontamination system vendor before January 29, 1999.

- Title I design for the characterization system is complete.

- A presentation was made to representatives of FETC-DDFA and Consumers Energy on the potential demonstration of the system at the Big Rock Point Decommissioning Project. Schedule acceleration opportunities are being pursued to perform the demonstration and initial deployment at Big Rock Point in September 1999.

\section{Accomplishments and technical progress}

\section{Literature Search to Determine Pipe Remediation Problem Set}

Rough order-of-magnitude quantities were obtained from Hanford and Fernald, including 150,000 $\mathrm{m}^{3}$ of pipe at Hanford and $5,880 \mathrm{~m}^{3}$ of pipe at Fernald. Obtaining quantities from other DOE operations offices would require a significant level of effort; therefore, FIU-HCET and the Deactivation and Decommissioning Focus Area (DDFA) decided that acquiring the additional information would not be cost-effective and concluded that significant volumes of pipe exist to warrant the continuation of the project.

\section{Determine Applicable Regulatory Policies and Procedures}

The list of regulations that govern the fabrication and operation of the pipe decontamination and characterization system was compiled. This list was given to the potential technology vendors to aid in proposal development, design, equipment fabrication, and system evaluation.

\section{Review of Decontamination and Characterization Technologies}

The review and collection of data for possible decontamination and characterization options for large-bore pipe are complete. Based on the information reviewed, an initial screening method used for pipe decontamination technologies was developed and implemented. The initial criteria include the technology's ability to meet the required clean, near-white metal surface finish ${ }^{1}$ on the interior or exterior of a pipe and the system's potential to be developed into a field mobile system. Seventeen decontamination technologies were evaluated as part of the initial screening process. Of the technologies screened, six technologies were selected for further evaluation; these six were then narrowed to one technology: grit blasting.

\footnotetext{
1 "A cleaned, near-white surface, when viewed without magnifications, shall be free of all visible oil, grease, dirt, dust, mill scale, rust, paint and oxides, corrosion products, and other foreign matter, except for staining. Staining shall be limited to no more than 5 percent of each square inch of the surface area and may consist of light shadows, slight streaks, or minor discolorations caused by rust stains, mill scale stains, or previously applied paint stains." (Structural Steel Painting Council, 1991, Surface Preparation Specifications, Structural Steel Painting Council, Pittsburgh, PA, pp. 53-56.)
} 
The literature survey of technologies capable of characterizing the interior and exterior of large-bore pipe is complete, and the resulting list detailing 21 technologies was prepared.

\section{Design and Fabricate Decontamination System}

Delong Equipment Company was selected to design, fabricate, and perform proof of principle testing of the decontamination module. The primary design difficulties involve laying out the system to fit into transportation containers. All critical issues have been resolved, and the system design indicates the performance specifications will be met.

Title I and Title II design are complete. Title III design is currently being performed. The transportation container for the ventilation system is currently being fabricated.

\section{Design and Fabricate Characterization System}

Canberra, Inc., was selected as contractor to supply the characterization module. A kick-off meeting was held on August 28,1998. Title I design was completed on November 30, 1998. Title II design is currently underway.

\section{Assessment of current status}

- The activities planned for completion are on schedule. The operations and maintenance procedures for the decontamination system are delayed due to revisions to the blast lance for pipe internal blasting. These procedures are not on a critical path for the completion of the decontamination system and will be available prior to the required date for use in the completion of milestone D017-M3.

- The procurement of the transportation container has a delivery schedule of April 2,1999. This delivery schedule will impact the completion of the decontamination system. The decontamination system is not on the critical path to meet the system deployment date; therefore, the schedule impact is minimal.

- The delivery of the conveyor for the characterization system has impacted the completion of the Title II design. The review and revision time periods for Title II will be shortened to minimize the schedule impact.

\section{Plans for the next two months}

- The transportation container for the decontamination system will be designed and fabricated. The procurement process began January 14, 1999, with delivery scheduled for April 2, 1999.

- The transportation trailers for all system components will be designed and fabricated. The procurement began on January 15, 1999, with delivery scheduled for March 31, 1999.

- The material run-off for the decontamination system will be complete. Material run-off is scheduled for February 12, 1999.

- Title II design for the characterization system will be complete.

- The air compressor and generator will be specified, and the procurement will be initiated. 


\section{FIU-HCET collaborator}

Joe Boudreaux, (423) 220-8844 


\title{
In-Situ Pipe Decontamination System
}

\author{
Project Number: HCET-1999-D041
}

\section{Project objectives}

The deactivation of radiologically contaminated facilities in many cases requires the characterization and decontamination of piping systems. There exists within the Department of Energy (DOE) inventory several thousand miles of piping and ductwork from facilities throughout the United States. The pipelines were used to move several types of contaminated fluids from one area to another within these facilities. The ductwork moved air within the facilities through ventilation systems. In-situ pipe decontamination options are limited; most commercial systems use highpressure water to clean the pipe internals. High-pressure water generates large volumes of wastewater, which requires treatment, and in many cases is not aggressive enough to remove heavy scale and contaminants.

The goal of this subtask is to develop a low-cost and efficient system for in-situ decontamination of pipes, which does not release contaminants into the environment or generate secondary waste.

The objectives of the project are the following:

- Determine performance factors for the decontamination system.

- Select the most capable technology for decontaminating in-situ pipes.

- Incorporate an efficient filtration system to prevent release of contaminants or generation of waste.

- Perform technology enhancement/integration to accommodate horizontal, straight, circular, and rectangular piping and ducting sections.

- Perform a cost-benefit analysis.

- Fabricate a prototype system and assess its performance.

Major milestones

\begin{tabular}{|c|l|l|l|}
\hline $\begin{array}{c}\text { Milestone } \\
\text { No. }\end{array}$ & \multicolumn{1}{|c|}{$\begin{array}{c}\text { Milestone } \\
\text { Description }\end{array}$} & \multicolumn{1}{|c|}{ Completion Criteria } & \multicolumn{1}{|c|}{ Status } \\
\hline D041-M1 & $\begin{array}{l}\text { Technology selection } \\
\text { complete }\end{array}$ & $\begin{array}{l}\text { Determine optimum technology to } \\
\text { deploy considering production rate, } \\
\text { decontamination factor, and safety } \\
\text { factors }\end{array}$ & $\begin{array}{l}\text { Scheduled } \\
\text { completion 1/29/99 }\end{array}$ \\
\hline D041-M2 & Design plan complete & $\begin{array}{l}\text { Peer review of design plan complete } \\
\text { and approved }\end{array}$ & $\begin{array}{l}\text { Scheduled } \\
\text { completion 2/5/99 }\end{array}$ \\
\hline D041-M3 & Design complete & $\begin{array}{l}\text { Peer review of final design drawings } \\
\text { and the cost to complete approved }\end{array}$ & $\begin{array}{l}\text { Scheduled } \\
\text { completion 6/11/99 }\end{array}$ \\
\hline D041-M4 & $\begin{array}{l}\text { Prototype system } \\
\text { complete and } \\
\text { demonstration test plan } \\
\text { developed }\end{array}$ & $\begin{array}{l}\text { Fabricated prototype system ready for } \\
\text { tests and demonstration test plan } \\
\text { approved }\end{array}$ & $\begin{array}{l}\text { Scheduled } \\
\text { completion 10/15/99 }\end{array}$ \\
\hline
\end{tabular}




\section{Significant events}

- The initial draft of the design plan has been submitted for Florida International University's Hemispheric Center for Environmental Technology (FIU-HCET) internal peer review. Expected completion date is $2 / 5 / 99$.

- From the list of candidate technologies identified in the December MPR, grit blasting was chosen as the technology to integrate into the system..

\section{Accomplishments and technical progress}

- A technical note on characterization of alpha contamination inside pipes was prepared for submission to Health Physics Journal.

- Candidate technologies for in-situ decontamination of pipes were screened and a list completed. (See December MPR Appendix A.)

\section{Assessment of current status}

This is the first year of a two-year project. The project is currently on schedule, and no issues impacting design or deployment have been identified to date.

\section{Plans for the next two months}

- Incorporate comments from the review and finalize design plan by February 5, 1999.

- A commercial vendor to fabricate and deploy the system will be selected by the end of March 1999.

\section{FIU-HCET collaborators}

Stan Vallidum, (305) 348-6554

S.K. Dua, (305) 348-1640 


\title{
Deactivation and Decommissioning Technology Opportunities for Non-Power NRC-Licensed Sites
}

\author{
Project Number: HCET-1999-D042
}

\section{Project objectives}

The Nuclear Regulatory Commission's (NRC) Operator Licensing Tracking System (OLTS) lists approximately 5,000 NRC-licensed operators of nuclear facilities in the United States. At the end of their useful life, power and non-power nuclear facilities must be deactivated and decommissioned. The use of appropriate deactivation and decommissioning (D\&D) technologies can enhance the safety, efficiency, and cost-effectiveness of cleanup operations.

Over the next 10 years, approximately 34 NRC-licensed non-power reactors (NPR) will begin the process of deactivation and decommissioning. Project managers at these sites will be faced with the challenge of selecting safe, cost-effective environmental technologies for achieving their remediation goals. Florida International University's Hemispheric Center for Environmental Technology (FIU-HCET), with its knowledge and expertise in environmental technologies and the D\&D process, will accomplish the following:

- Assess the needs of these NRC-licensed non-power reactor sites.

- Identify opportunities for the fielding of technologies that have been proven safe and effective through research, development, and testing sponsored by the DOE's Office of Science and Technology.

\section{Major milestones}

\begin{tabular}{|c|l|l|l|}
\hline Milestone No. & Milestone Description & \multicolumn{1}{|c|}{ Completion Criteria } & \multicolumn{1}{c|}{ Status } \\
\hline D042-M1 & $\begin{array}{l}\text { Report on site licensing, } \\
\text { decommissioning, and } \\
\text { participation information }\end{array}$ & $\begin{array}{l}\text { List of NPRs indicating nature of } \\
\text { operation, license expiration date, } \\
\text { decommissioning schedule, and } \\
\text { willingness of the site to participate in the } \\
\text { study. }\end{array}$ & $\begin{array}{l}\text { On schedule for } \\
\text { completion by } \\
\text { February 28,1999. }\end{array}$ \\
\hline D042-M2 & $\begin{array}{l}\text { DOE-FIU-HCET decision } \\
\text { to proceed and NPR sites to } \\
\text { be studied }\end{array}$ & $\begin{array}{l}\text { Based on level of participation, a decision } \\
\text { by DOE and FIU-HCET on whether to } \\
\text { proceed with the project. }\end{array}$ & $\begin{array}{l}\text { Scheduled for May } \\
15,1999 .\end{array}$ \\
\hline D042-M3 & Site needs assessment & $\begin{array}{l}\text { Identification of key problem sets facing } \\
\text { each NPR scheduled for } \\
\text { decommissioning. }\end{array}$ & $\begin{array}{l}\text { Scheduled to begin } \\
\text { April } 1999 .\end{array}$ \\
\hline D042-M4 & Technological solutions & $\begin{array}{l}\text { Identification of candidate technologies } \\
\text { for addressing problem sets identified in } \\
\text { milestone 3. }\end{array}$ & $\begin{array}{l}\text { Scheduled to begin } \\
\text { July 1999. }\end{array}$ \\
\hline D042-M5 & Final report & $\begin{array}{l}\text { Report summarizing problem sets and } \\
\text { potential technological solutions } \\
\text { submitted to DoE and distributed to NPR } \\
\text { sites and technology providers. }\end{array}$ & $\begin{array}{l}\text { To be completed } \\
\text { before October 31, } \\
1999 .\end{array}$ \\
\hline
\end{tabular}




\section{Significant events}

- Contact information for managers on 40 of the 43 NPRs in the survey has been obtained either from the Internet web sites of the facilities or through direct telephone contact (see Appendix A). Survey information is being collected via telephone interviews.

\section{Accomplishments and technical progress}

- To date, six NPR managers have been contacted, and four have committed to participating in the survey and providing site assessment and decommissioning information on their facilities.

\section{Assessment of current status}

- The project is proceeding on schedule, and planned milestones will be met.

\section{Plans for the next two months}

- The remaining site managers at candidate NPRs listed in Appendix A will be contacted and informed of the purpose of this subtask and the potential benefits of the results of the study to their decommissioning efforts. They will also be asked to indicate their interest in participating in the study and be apprised of the information they will be required to provide if they wish to participate.

\section{FIU-HCET collaborator}

Robert Tucker, (305) 348-6181 


\section{Appendix A: U.S. Nuclear Non-power Reactors}

\begin{tabular}{|c|c|c|c|c|}
\hline 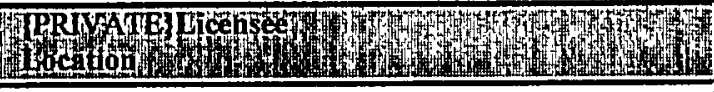 & 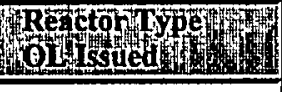 & 6) & M & 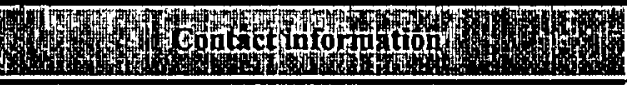 \\
\hline $\begin{array}{l}\text { Aerotest } \\
\text { San Ramon, CA }\end{array}$ & $\begin{array}{l}\text { Triga (Indus) } \\
07 / 02 / 1965\end{array}$ & $\begin{array}{l}\text { OL } \\
50-228\end{array}$ & $\mathrm{R}-98$ & Name Not Available \\
\hline $\begin{array}{l}\text { Armed Forces Radiobiology Research Inst. } \\
\text { Bethesda, MD }\end{array}$ & $\begin{array}{l}\text { Triga } \\
06 / 26 / 1962\end{array}$ & $\begin{array}{l}\mathrm{OL} \\
50-170\end{array}$ & R-84 & Public Affairs Officer: pao@mx.afrri.usuhs.mil \\
\hline $\begin{array}{l}\text { Cornell University } \\
\text { Ithaca, NY }\end{array}$ & $\begin{array}{l}\text { Zero Power } \\
12 / 11 / 1962\end{array}$ & $\begin{array}{c}\mathrm{OL} \\
50-97\end{array}$ & $\mathrm{R}-89$ & $\begin{array}{c}\text { Mr. Martin Moravek } \\
\text { Gamma Cell Operations } \\
\text { phone: (607)255-9420 } \\
\text { E-mail: mm129@comell.edu }\end{array}$ \\
\hline $\begin{array}{l}\text { Cornell University } \\
\text { Ithaca, NY }\end{array}$ & $\begin{array}{l}\text { Triga Mark II } \\
01 / 11 / 1962\end{array}$ & $\begin{array}{l}\text { OL } \\
50-157\end{array}$ & $\mathrm{R}-80$ & $\begin{array}{l}\text { Mr. Paul I. Craven } \\
\text { TRIGA Reactor Operations } \\
\text { phone: (607)255-2370 } \\
\text { E-mail: pic1@comell.edu }\end{array}$ \\
\hline $\begin{array}{l}\text { Dow Chemical Company } \\
\text { Midland, MI }\end{array}$ & $\begin{array}{l}\text { Triga } \\
07 / 03 / 1967\end{array}$ & $\begin{array}{c}\mathrm{OL} \\
50-264\end{array}$ & R-108 & $\begin{array}{c}\text { Dow Chemical Customer Information Group } \\
690 \text { Building } \\
\text { Midland, Michigan } \\
\text { U.S.A. - } 48640 \\
\text { Phone: } 1-800-258-2436 \\
\text { Fax: } 517-832-1465\end{array}$ \\
\hline $\begin{array}{l}\text { General Atomics Mark I } \\
\text { San Diego, CA }\end{array}$ & $\begin{array}{l}\text { Triga Mark I } \\
05 / 03 / 1958\end{array}$ & $\begin{array}{c}\mathrm{OL} \\
50-89\end{array}$ & $\mathrm{R}-38$ & $\begin{array}{l}\frac{\text { www.gat.com }}{\text { triga@gat.com }} \\
\text { Phone: }(619) 457-8820 \\
\text { fax (619) 457-8786 }\end{array}$ \\
\hline $\begin{array}{l}\text { General Electric Company } \\
\text { Pleasanton, CA }\end{array}$ & $\begin{array}{l}\text { Nuclear Test } \\
10 / 31 / 1957\end{array}$ & $\begin{array}{c}\mathrm{OL} \\
50-73\end{array}$ & $\mathrm{R}-33$ & Name Not Available \\
\hline
\end{tabular}

HCET /anuary 1999 Monthly Progress Reports 


\begin{tabular}{|c|c|c|c|c|}
\hline $\begin{array}{l}\text { Georgia Institute of Technology } \\
\text { Atlanta, GA }\end{array}$ & $\begin{array}{l}\text { Heavy Water } \\
12 / 29 / 1964\end{array}$ & $\begin{array}{c}\mathrm{OL} \\
50-160\end{array}$ & R-97 & $\begin{array}{l}\frac{\text { dwayne.blaylock@nnrc.gatech.edu }}{\text { for general information, and }} \\
\text { arlene.smith@nnrc.gatech.edu } \\
\text { for administrative queries }\end{array}$ \\
\hline $\begin{array}{l}\text { Idaho State University } \\
\text { Pocatello, ID }\end{array}$ & $\begin{array}{l}\text { AGN-201\#103 } \\
10 / 11 / 1967\end{array}$ & $\begin{array}{c}\mathrm{OL} \\
50-284\end{array}$ & $\mathrm{R}-110$ & $\begin{array}{c}\text { Dr. Frank Harmon } \\
\text { Department of Physics } \\
\text { Idaho State University } \\
\text { Campus Box } 8106 \\
\text { Pocatello, ID. } 83209-8106 \\
\text { Phone (208) 236-2350 } \\
\text { FAX (208) 236-4649 } \\
\text { E-mail: icsa@apollo.physics.isu.edu }\end{array}$ \\
\hline $\begin{array}{l}\text { lowa State University } \\
\text { Ames, IA }\end{array}$ & $\begin{array}{l}\text { Argonaut } \\
10 / 16 / 1959\end{array}$ & $\begin{array}{l}\mathrm{OL} \\
50-116\end{array}$ & R-59 & $\begin{array}{l}\text { Mechanical Engineering Department } \\
2025 \text { H. M. Black Engineering Building } \\
\text { lowa State University } \\
\text { Ames, Iowa 50011-2161 } \\
\text { Phone: } 515-294-1423 \\
\text { Fax: } 515-294-3261 \\
\text { E-mail: isume@iastate.edu }\end{array}$ \\
\hline $\begin{array}{l}\text { Kansas State University } \\
\text { Manhattan, KS }\end{array}$ & $\begin{array}{l}\text { Triga } \\
10 / 16 / 1962\end{array}$ & $\begin{array}{l}\mathrm{OL} \\
50-188\end{array}$ & $\mathrm{R}-88$ & $\begin{array}{c}\text { Brendan Ryan, } \\
\text { Reactor Facility Manager, } \\
\text { Phone: (785)-532-6657 } \\
\text { E-mail: ibryan@ne.ksu.edu, }\end{array}$ \\
\hline $\begin{array}{l}\text { Manhattan College } \\
\text { Bronx, NY }\end{array}$ & $\begin{array}{l}\text { Tank } \\
03 / 24 / 1964\end{array}$ & $\begin{array}{c}\mathrm{OL} \\
50-199\end{array}$ & $\mathrm{R}-94$ & $\begin{array}{c}\text { Manhattan College } \\
\text { Riverdale, New York city, } \\
\text { N.Y. 10471 } \\
\text { 1-800-mc2-xcel }\end{array}$ \\
\hline $\begin{array}{l}\text { Massachusetts Institute of Technology } \\
\text { Cambridge, MA }\end{array}$ & $\begin{array}{l}\text { HWR Reflected } \\
06 / 09 / 1958\end{array}$ & $\begin{array}{c}\mathrm{OL} \\
50-20\end{array}$ & $\mathrm{R}-37$ & nrl-www@mit.edu \\
\hline
\end{tabular}




\begin{tabular}{|c|c|c|c|c|}
\hline $\begin{array}{l}\text { National Institute of Standards \& Technology } \\
\text { Gaithersburg, MD }\end{array}$ & $\begin{array}{l}\text { Nuclear Test } \\
06 / 30 / 1970\end{array}$ & $\begin{array}{c}\mathrm{OL} \\
50-184\end{array}$ & TR-5 & $\begin{array}{l}\text { NIST Public Inquiries Unit, } \\
\text { Admin. A903, NIST, } \\
\text { Gaithersburg, MD 20899-0001, } \\
\text { Phone: (301) 975-NIST, } \\
\text { E-mail:inquiries@nist.gov } \\
\text { Katharine B. Gebbie, Director } \\
\text { Tel } 3019754201 \\
\text { Fax 301 975 3038 } \\
\text { E-mail: katharine.gebbie@nist.gov }\end{array}$ \\
\hline $\begin{array}{l}\text { North Carolina State } \\
\text { University } \\
\text { Raleigh, NC }\end{array}$ & $\begin{array}{l}\text { Pulstar } \\
08 / 25 / 1972\end{array}$ & $\begin{array}{c}\text { OL } \\
50-297\end{array}$ & $R-120$ & $\begin{array}{c}\text { Prof. Donald J. Dudziak } \\
\text { CAPES- Center for Applied Plasma Engineering and } \\
\text { Sciences. } \\
\text { Chairperson-Nuclear Eng. dept } \\
\text { Ph: } 919-515-6289, \\
\text { Fax: } 919-515-6305 \\
\text { E-mail: dudziak@eos.ncsu.edu }\end{array}$ \\
\hline $\begin{array}{l}\text { Ohio State University } \\
\text { Columbus, OH }\end{array}$ & $\begin{array}{l}\text { Pool } \\
02 / 24 / 1961\end{array}$ & $\begin{array}{c}\mathrm{OL} \\
50-150\end{array}$ & $\mathrm{R}-75$ & $\begin{array}{l}\text { Don W. Miller, } \\
\text { Chairperson- Nuclear Eng. dept. } \\
\text { 1075 Robinson Laboratory } \\
206 \text { W 18th Ave } \\
\text { Columbus, OH 43210 } \\
\text { Phone: 614-292-7979 } \\
\text { Fax:614-292-3163 } \\
\text { E-mail: miller.68@osu.edu }\end{array}$ \\
\hline $\begin{array}{l}\text { Oregon State University } \\
\text { Corvallis, OR }\end{array}$ & $\begin{array}{l}\text { Triga Mark II } \\
03 / 07 / 1967\end{array}$ & $\begin{array}{c}\mathrm{OL} \\
50.243\end{array}$ & R-106 & $\begin{array}{c}\text { Stephen E. Binney - Director } \\
\text { (541) 737-2344 } \\
\text { binneys@rc.orst.edu } \\
\text { Contact person for all new projects. } \\
\text { Office Manager-Donna Dalton } \\
\text { (541) 737-7039 } \\
\text { E-mail: daltondk@rc.orst.edu } \\
\text { Appointments, initial contact \& general query. }\end{array}$ \\
\hline
\end{tabular}




\begin{tabular}{|c|c|c|c|c|}
\hline $\begin{array}{l}\text { Pennsylvania State University } \\
\text { University Park, PA }\end{array}$ & $\begin{array}{l}\text { Triga } \\
07 / 08 / 1955\end{array}$ & $\begin{array}{l}\mathrm{OL} \\
50-5\end{array}$ & $\mathrm{R}-2$ & $\begin{array}{c}\text { Sears C Frederick - Director } \\
\text { Radiation Science and Engineering Center } \\
\text { Address: 0101 BREAZEALE NUCLEAR } \\
\text { REACTOR } \\
\text { UNIVERSITY PARK, PA } 16802 \\
\text { phone: } 8148656351 \\
\text { fax: } 8148634840 \\
\text { E-mail: cfs7@psu.edu }\end{array}$ \\
\hline $\begin{array}{l}\text { Purdue University } \\
\text { West Lafayette, IN }\end{array}$ & $\begin{array}{l}\text { Lockheed } \\
08 / 16 / 1962\end{array}$ & $\begin{array}{c}\mathrm{OL} \\
50-182\end{array}$ & R-87 & $\begin{array}{c}\text { Prof. Bement, } \\
\text { Head of nuclear Eng. dept. } \\
\text { phone : } 3174945742 \\
\text { E-mail: bement@ecn.purdue.edu }\end{array}$ \\
\hline $\begin{array}{l}\text { Reed College } \\
\text { Portland, OR }\end{array}$ & $\begin{array}{l}\text { Triga Mark I } \\
07 / 02 / 1968\end{array}$ & $\begin{array}{c}\mathrm{OL} \\
50-288\end{array}$ & $\mathrm{R}-112$ & $\begin{array}{l}\text { Stephen Frantz - Director } \\
\text { Reed College Reactor } \\
\text { 3203 Southeast Woodstock Blvd. } \\
\text { Portland, Oregon } 97202-8199 \\
\text { Voice: } 503-777-7222 \\
\text { Fax: 503-777-7274 } \\
\text { E-mail: sfrantz@aone.com } \\
\text { E-mail: reactor@reed.edu }\end{array}$ \\
\hline $\begin{array}{l}\text { Rensselaer Polytechnic Institute } \\
\text { Troy, NY }\end{array}$ & $\begin{array}{l}\text { Critical Assembly } \\
07 / 03 / 1964\end{array}$ & $\begin{array}{c}\mathrm{OL} \\
50-225\end{array}$ & $C X-22$ & $\begin{array}{c}\text { Dr. George Xu } \\
\text { Phone: (518) 276-4014; } \\
\text { FAX: (518) 276-4832. } \\
\text { E-mail: xug2@rpi.edu }\end{array}$ \\
\hline $\begin{array}{l}\text { Rhode Island Atomic Energy Commission } \\
\text { Narragansett, RI }\end{array}$ & $\begin{array}{l}\text { GE Pool } \\
07 / 21 / 1964\end{array}$ & $\begin{array}{c}\mathrm{OL} \\
50-193\end{array}$ & R-95 & $\begin{array}{c}\text { Terry - Director } \\
\text { Rhode Island Atomic Energy Commission } \\
\text { Rhode Island Nuclear Science Center } \\
\text { 16 Reactor Road } \\
\text { Narragansett, RI 02882-1197 } \\
\text { Fax: 401-782-4201 } \\
\text { Tehanttehan@gsosun1.gso.uri.edu }\end{array}$ \\
\hline $\begin{array}{l}\text { State University of New York (Buffalo) } \\
\text { Buffalo, NY }\end{array}$ & $\begin{array}{l}\text { Pulstar } \\
03 / 24 / 1961\end{array}$ & $\begin{array}{c}\mathrm{OL} \\
50-57\end{array}$ & $\mathrm{R}-77$ & \begin{tabular}{|c|} 
Mark S. Adams \\
Occupational and Environmental Safety Services \\
(OES) \\
Operations Manager, BMRC \\
Phone:716 829-3301 or 716 645-6844 \\
Richard L. Jones \\
Phone: 716 829-3281.
\end{tabular} \\
\hline
\end{tabular}




\begin{tabular}{|c|c|c|c|c|}
\hline $\begin{array}{l}\text { Texas A\&M University } \\
\text { College Station, TX }\end{array}$ & $\begin{array}{l}\text { AGN-201M \#106 } \\
08 / 26 / 1957\end{array}$ & $\begin{array}{c}\text { OL } \\
50-59\end{array}$ & R-23 & $\begin{array}{c}\text { Dr. Waltar } \\
\text { Chairperson- Texas A\&M Nuclear Eng. Dept } \\
\text { 129 Zachry Engineering Center } \\
\text { College Station, TX 77843-3133 } \\
\text { Telephone: 409-845-7078 } \\
\text { Telefax: 409-845-6443 } \\
\text { E-mail: waltar@trinity.tamu.edu }\end{array}$ \\
\hline $\begin{array}{l}\text { Texas A\&M University } \\
\text { College Station, TX }\end{array}$ & $\begin{array}{l}\text { Triga } \\
12 / 07 / 1961\end{array}$ & $\begin{array}{c}\mathrm{OL} \\
50-128\end{array}$ & R-128 & $\begin{array}{c}\text { Dr. Waltar } \\
\text { Chairperson- Texas A\&M Nuclear Eng. Dept } \\
\text { 129 Zachry Engineering Center } \\
\text { College Station, TX 77843-3133 } \\
\text { Telephone: 409-845-7078 } \\
\text { Telefax: 409-845-6443 } \\
\text { E-mail: waltar@trinity.tamu.edu }\end{array}$ \\
\hline $\begin{array}{l}\text { U.S. Geological Survey } \\
\text { Denver, CO }\end{array}$ & $\begin{array}{l}\text { Triga Mark I } \\
02 / 24 / 1969\end{array}$ & $\begin{array}{c}\mathrm{OL} \\
50-274\end{array}$ & R-113 & $\begin{array}{l}\text { Douglas R. Posson - Director } \\
\text { MS 150, PO Box 25046, } \\
\text { Denver Federal Center, Bldg. 53, } \\
\text { Denver, CO 80225-0046 } \\
\text { Phone: 303-236-5900 x303 } \\
\text { E-mail: dposson@usgs.gov }\end{array}$ \\
\hline $\begin{array}{l}\text { University of Arizona } \\
\text { Tucson, AZ }\end{array}$ & $\begin{array}{l}\text { Triga Mark I } \\
\text { 12/05/1958 }\end{array}$ & $\underset{50-113}{\mathrm{OL}}$ & $\mathrm{R}-52$ & $\begin{array}{c}\text { John Williams- - Director } \\
\text { Phone: 520-621-9729 } \\
\text { john-williams@ns.arizona.edu } \\
\text { (igw@engr.arizona.edu ) } \\
\text { Gould-Simpson 1013 } \\
\text { Tucson, Arizona 85721-0077 } \\
\text { Tel: 1-520-621-4064 } \\
\text { FAX: } 1-520-621-1364 \\
\text { Internet: arl@arl.arizona.edu }\end{array}$ \\
\hline $\begin{array}{l}\text { University of Calif } \cdots \text {, } \\
\text { Irvine, Irvine, } \mathrm{CA}\end{array}$ & $\begin{array}{l}\text { Triga Mark I } \\
\text { 11/24/1969 }\end{array}$ & $\underset{50-326}{\mathrm{OL}}$ & R-116 & $\begin{array}{c}\text { Dr. George E. Miller } \\
\text { Reactor Supervisor } \\
\text { Phone:(714) 824-6649 } \\
\text { E-mail:gemiller@uci.edu }\end{array}$ \\
\hline
\end{tabular}




\begin{tabular}{|c|c|c|c|c|}
\hline $\begin{array}{l}\text { University of Florida } \\
\text { Gainesville, FL }\end{array}$ & $\begin{array}{l}\text { Argonaut } \\
05 / 21 / 1959\end{array}$ & $\begin{array}{c}\mathrm{OL} \\
50-83\end{array}$ & R-56 & $\begin{array}{c}\text { James S. Tulenko } \\
\text { Professor and Chairman } \\
\text { E-mail: tulenko@ufl.edu } \\
\text { 202 Nuclear Sciences Center } \\
\text { P.O. BOX } 118300 \\
\text { Gainesville, Fl 32611-8300 } \\
\text { Voice: (352) 392-1401 } \\
\text { Fax: (352) 392-3380 } \\
\text { Email:deptdm@serverl.nuceng.ufl.edu }\end{array}$ \\
\hline $\begin{array}{l}\text { University of Illinois } \\
\text { Urbana, IL }\end{array}$ & $\begin{array}{l}\text { Triga } \\
07 / 22 / 1969\end{array}$ & $\begin{array}{l}\mathrm{OL} \\
50-151\end{array}$ & $\mathrm{R}-115$ & $\begin{array}{c}\text { Dr. Barclay G. Jones, } \\
\text { Head Department of Nuclear Eng. } \\
214 \text { Nuclear Engineering Laboratory } \\
\text { University of Illinois at Urbana-Champaign } \\
\text { 103 South Goodwin Avenue } \\
\text { Urbana, Illinois 61801-2984 } \\
\text { Phone: (217) 333-2295 } \\
\text { Fax: (217) 333-2906 } \\
\text { E-mail: bgiones@uiuc.edu }\end{array}$ \\
\hline $\begin{array}{l}\text { University of Lowell } \\
\text { Lowell, MA }\end{array}$ & $\begin{array}{l}\text { GE Pool } \\
12 / 24 / 1974\end{array}$ & $\begin{array}{c}\mathrm{OL} \\
50-223\end{array}$ & $R-125$ & $\begin{array}{c}\text { Lee H. Bettenhausen - Adjunct Professor, } \\
\text { Director-University of Massachusetts Lowell } \\
\text { Research Reactor. } \\
\text { Leo Bobek } \\
\text { Radiation Laboratory, Reactor Supervisor } \\
\text { Phone:978 934-3365 } \\
\text { Email: Leo Bobek@uml.edu }\end{array}$ \\
\hline $\begin{array}{l}\text { University of Maryland } \\
\text { College Park, MD }\end{array}$ & $\begin{array}{l}\text { Triga } \\
10 / 14 / 1960\end{array}$ & $\begin{array}{l}\mathrm{OL} \\
50-166\end{array}$ & $\mathrm{R}-70$ & $\begin{array}{c}\text { Vincent Adams } \\
\text { Manager-Reactor Facilities } \\
\text { A. J. Clark School of Engineering } \\
\text { 2135 Chemical and Nuclear Eng. bldg. } \\
\text { Phone: (301) 405-5207 } \\
\text { Fax:(301) 314-2029 } \\
\text { E-mail: via@umdacc.umd.edu }\end{array}$ \\
\hline $\begin{array}{l}\text { University of Michigan } \\
\text { Ann Arbor, MI }\end{array}$ & $\begin{array}{l}\text { Pool } \\
09 / 13 / 1957\end{array}$ & $\begin{array}{c}\mathrm{OL} \\
50-2\end{array}$ & $\mathrm{R}-28$ & $\begin{array}{c}\text { John C. Lee - Interim Director } \\
\text { Phone: (734)764-6213 } \\
\text { Philip A. Simpson - Acting Reactor Mgr. (734)764- } \\
6221 \\
\text { Telephone: (734) 764-6221 } \\
\text { Fax: (734) 936-1571 } \\
\text { E-Mail: phils@umich.edu }\end{array}$ \\
\hline
\end{tabular}




\begin{tabular}{|c|c|c|c|c|}
\hline $\begin{array}{l}\text { University of Missouri/Rolla } \\
\text { Rolla, MO }\end{array}$ & $\begin{array}{l}\text { Pool } \\
11 / 21 / 1961\end{array}$ & $\begin{array}{l}\mathrm{OL} \\
50-123\end{array}$ & R-79 & $\begin{array}{l}\text { Nuclear Engineering. } \\
\text { Email: gmueller@umr.edu } \\
\text { Department of Chemistry: } \\
\text { University of Missouri - Rolla } \\
\text { Rolla, MO 65409 } \\
\text { Phone: (573) } 341-4420 \\
\text { Fax: (573) 341-6033 } \\
\text { Email: chem@umr.edu } \\
\end{array}$ \\
\hline $\begin{array}{l}\text { University of Missouri/ } \\
\text { Columbia, MO }\end{array}$ & $\begin{array}{l}\text { Tank } \\
10 / 11 / 1966\end{array}$ & $\begin{array}{c}\text { OL } \\
50-186\end{array}$ & $R-103$ & $\begin{array}{l}\text { William H. Miller - Group leader } \\
\text { Professor \& Chairman } \\
\text { E2433 Eng. Bldg. East } \\
\text { University of Missouri - Columbia } \\
\text { Columbia 65211 } \\
\text { Phone: (573) 882-9692 } \\
\text { E-mail: whmiller@ecn.missouri.edu }\end{array}$ \\
\hline $\begin{array}{l}\text { University of New Mexico } \\
\text { Albuquerque, NM }\end{array}$ & $\begin{array}{l}\text { AGN-201M\#112 } \\
09 / 17 / 1966\end{array}$ & $\begin{array}{c}\mathrm{OL} \\
50-252\end{array}$ & R-102 & \begin{tabular}{|c|} 
Mohamed S El-Genk \\
Regents Professor \& Director \\
Institute for Space and Nuclear Power Studies \\
Department of Chemical \& Nuclear Engineering \\
University of New Mexico \\
Tel: (505)-277-5442/0446, \\
Fax: (505)-277-2814 \\
mgenk@unm.edu
\end{tabular} \\
\hline $\begin{array}{l}\text { University of Texas } \\
\text { Austin, TX }\end{array}$ & $\begin{array}{l}\text { Triga Mark II } \\
01 / 17 / 1992\end{array}$ & $\begin{array}{c}\mathrm{OL} \\
50-602\end{array}$ & R-92 & $\begin{array}{c}\text { Dr. Felib Y. Iskander, } \\
\text { Nuclear Engineering Teaching Laboratory } \\
\text { Mail Stop R9000 Austin, } \\
\text { Texas } 78712 \\
\text { Tel (512) 471-5787 } \\
\text { Fax (512) 471-4589 } \\
\text { Email - F.Iskander@mail.utexas.edu }\end{array}$ \\
\hline $\begin{array}{l}\text { University of Utah/Triga } \\
\text { Salt Lake City, UT }\end{array}$ & $\begin{array}{l}\text { Triga Mark I } \\
09 / 30 / 1975\end{array}$ & $\begin{array}{c}\mathrm{OL} \\
50-407\end{array}$ & R-126 & $\begin{array}{c}\text { Dr. David M. Slaughter-Director } \\
\text { RM. 104, } 160 \text { South Central Campus Dr. } \\
\text { Salt Lake City, Utah } 841112 \\
\text { Tel: } 801-581-8499 \\
\text { Email - davids@scottie.mech.utah.edu }\end{array}$ \\
\hline
\end{tabular}




\begin{tabular}{|c|c|c|c|c|}
\hline $\begin{array}{l}\text { University of Virginia } \\
\text { Charlottesville, VA }\end{array}$ & $\begin{array}{l}\text { Pool } \\
06 / 27 / 1960\end{array}$ & $\begin{array}{c}\mathrm{OL} \\
50-62\end{array}$ & $\mathrm{R}-66$ & $\begin{array}{c}\text { The Center for Electrochemical Science and } \\
\text { Engineering (CESE) } \\
\text { Department of Materials Science and Engineering } \\
\text { university of Virginia } \\
\text { Thornton Hall, } \\
\text { Charlottesville, Virginia 22903-2442 } \\
\text { Phone: (804) 982-5780 }\end{array}$ \\
\hline $\begin{array}{l}\text { University of Wisconsin } \\
\text { Madison, WI }\end{array}$ & $\begin{array}{l}\text { Triga } \\
11 / 23 / 1960\end{array}$ & $\begin{array}{c}\mathrm{OL} \\
50-156\end{array}$ & $\mathrm{R}-74$ & $\begin{array}{c}\text { Richard J. Cashwell, } \\
141 \text { Mechanical Engineering Building } \\
1513 \text { University Avenue } \\
\text { Madison, WI } 53706 \\
\text { Tel: } 608-262-3392 \\
\text { Fax: } 608-262-8590 \\
\text { E-mail: cashwell@engr.wisc.edu }\end{array}$ \\
\hline $\begin{array}{l}\text { Veterans Administration } \\
\text { Omaha, NE }\end{array}$ & $\begin{array}{l}\text { Triga } \\
06 / 26 / 1959\end{array}$ & $\underset{50-131}{\mathrm{OL}}$ & $\mathrm{R}-57$ & Name Not Available \\
\hline $\begin{array}{l}\text { Washington State University } \\
\text { Pullman, WA }\end{array}$ & $\begin{array}{l}\text { Triga } \\
03 / 06 / 1961\end{array}$ & $\begin{array}{l}\mathrm{OL} \\
50-27\end{array}$ & $\mathrm{R}-76$ & $\begin{array}{l}\text { Al Ruddy } \\
\text { Tel: } 509-335-4528 \\
\text { Tel: } 509-335-7592\end{array}$ \\
\hline $\begin{array}{l}\text { Worcester Polytechnic Institute } \\
\text { Worcester, MA }\end{array}$ & $\begin{array}{l}\text { GE } \\
12 / 16 / 1959\end{array}$ & $\begin{array}{c}\mathrm{OL} \\
50-134\end{array}$ & $\mathrm{R}-61$ & $\begin{array}{c}\text { Prof. Leo Bobek } \\
\text { Tel: 508-831-5276 } \\
\text { 508-831-5680 } \\
\text { Email: } \text { bobek@,wpi.edu }\end{array}$ \\
\hline
\end{tabular}

Note: Limited to nonpower reactors licensed to operate.

Source: Nuclear Regulatory Commission, http://www.nrc.gov/NRC/NUREGS/SR1350/V9/sr1350.html 


\title{
Life-Cycle Cost Analysis for Radioactively Contaminated Scrap Metal
}

\author{
Project Number: HCET-1999-D043
}

\section{Project objectives}

In September 1996, the Assistant Secretary of the U.S. Department of Energy's Office of Environmental Management (U.S. DOE-EM) issued a challenge to the DOE community that, to the degree that recycling is economically advantageous and protective of worker and public health, radioactively contaminated scrap metal (RSM) presently in storage, or projected to be generated by future EM activities, should be recycled.

Future deactivation and decommissioning (D\&D) of the DOE's surplus facilities is expected to generate more than 600,000 tons of metal and 23 million cubic meters of concrete. Already there are more than 400,000 tons of RSM from past D\&D activities temporarily stockpiled at DOE sites and pending disposition. There are also large quantities of RSM permanently buried at commercial and DOE-managed low-level waste (LLW) disposal facilities across the country. In total, it is estimated that more than 2,000,000 tons of RSM will be generated from the deactivation and decommissioning of radioactively contaminated facilities at the $\mathrm{DOE}$ and in the private sector.

Current waste disposal costing methodologies at DOE-managed waste disposal sites favor direct disposal of RSM in landfills over recycling. Studies commissioned by the DOE have shown that current rates for direct disposal of RSM are understated because they do not fully reflect all the costs associated with the full life-cycle of waste disposal, specifically the long-term maintenance and surveillance of disposal sites after they have been closed. A more accurate life-cycle cost analysis (LCCA) would certainly reflect higher costs for direct disposal and would lead to increased material recycling, resource recovery, and waste minimization - key goals of the DOE.

The objective of the subtask is the following:

- Update the methodology(s) currently used at DOE-managed waste disposal facilities for determining disposal costs for RSM to take into account all costs incurred over the entire lifecycle of the waste in order to demonstrate that recycling is a cost-competitive means for disposition of RSM.

\section{Major milestones}

\begin{tabular}{|c|l|l|l|}
\hline Milestone No. & Milestone Description & \multicolumn{1}{|c|}{ Completion Criteria } & \multicolumn{1}{c|}{ Status } \\
\hline D043-M1 & RSM inventory & $\begin{array}{l}\text { A summary of estimated current and } \\
\text { future RSM generated from D\&D } \\
\text { activities. }\end{array}$ & $\begin{array}{l}\text { The scheduled } \\
\text { completion date of } \\
2 / 1 / 99 \text { is revised } \\
\text { to 2/15/99. }\end{array}$ \\
\hline D043-M2 & $\begin{array}{l}\text { List of committed waste } \\
\text { site managers }\end{array}$ & $\begin{array}{l}\text { A list of waste site managers willing to } \\
\text { provide information necessary for } \\
\text { developing LCCAs. }\end{array}$ & $\begin{array}{l}\text { On schedule for } \\
\text { completion by } \\
2 / 1 / 99 .\end{array}$ \\
\hline D043-M3 & $\begin{array}{l}\text { Report to DOE on Phase 1 } \\
\text { (milestones 1 and 2) }\end{array}$ & $\begin{array}{l}\text { Summary of results of milestones 1 and } \\
\text { 2 and recommendation to DOE whether } \\
\text { to proceed. }\end{array}$ & $\begin{array}{l}\text { Scheduled for } \\
\text { completion 3/1/99 }\end{array}$ \\
\hline
\end{tabular}




\begin{tabular}{|c|l|l|l|}
\hline Milestone No. & Milestone Description & \multicolumn{1}{|c|}{ Completion Criteria } & \multicolumn{1}{|c|}{ Status } \\
\hline D043-M4 & $\begin{array}{l}\text { Decision on RSM disposal } \\
\text { sites and options to be } \\
\text { assessed }\end{array}$ & $\begin{array}{l}\text { List of waste disposal sites that will be } \\
\text { surveyed and disposal options for which } \\
\text { LCCAs will be developed. }\end{array}$ & $\begin{array}{l}\text { On schedule for } \\
\text { review 3/15/99 }\end{array}$ \\
\hline D043-M5 & $\begin{array}{l}\text { Documentation of NRC } \\
\text { and commercial means of } \\
\text { costing RSM disposition }\end{array}$ & $\begin{array}{l}\text { Comparative analysis of costing } \\
\text { methodology and factors used by } \\
\text { selected sites. }\end{array}$ & $\begin{array}{l}\text { Scheduled to } \\
\text { begin 3/99. }\end{array}$ \\
\hline D043-M6 & $\begin{array}{l}\text { Documentation of DOE } \\
\text { disposition }\end{array}$ & $\begin{array}{l}\text { Procedural outline of DOE's costing } \\
\text { methodology indicating variances with } \\
\text { other sites surveyed. }\end{array}$ & $\begin{array}{l}\text { Scheduled to } \\
\text { begin 4/99. }\end{array}$ \\
\hline D043-M7 & Life-Cycle Cost Analyses & $\begin{array}{l}\text { Revised LCCAs submitted to DOE } \\
\text { considering variances identified in } \\
\text { milestone 6 as well as ORNL's Life } \\
\text { Cycle Decision Methodology for RSM. }\end{array}$ & $\begin{array}{l}\text { Scheduled to } \\
\text { begin 3/99. }\end{array}$ \\
\hline D043-M8 & $\begin{array}{l}\text { Finalize LCCAs and update } \\
\text { DOE handbook }\end{array}$ & $\begin{array}{l}\text { Revised LCCAs incorporating DOE's } \\
\text { comments and procedures for LCCA } \\
\text { incorporated in DOE handbook. }\end{array}$ & $\begin{array}{l}\text { Scheduled to } \\
\text { begin 6/99. }\end{array}$ \\
\hline D043-M9 & Final report & $\begin{array}{l}\text { Final report documenting results of } \\
\text { project submitted to DOE. }\end{array}$ & Due 10/31/99. \\
\hline
\end{tabular}

\section{Significant events}

Progress toward completion of milestone 1 has been slowed by the delay in obtaining data on existing RSM inventories. Alternative arrangements are being made to obtain this information from the Association of Radioactive Metal Recyclers in Knoxville, Tennessee. Although milestone 1 will be delayed by approximately 2 weeks, this will not affect the March 1, 1999, delivery of FIUHCET's report to the US-DOE (milestone 3) summarizing the results of milestones 1 and 2 and recommendation to DOE whether to proceed.

\section{Accomplishments and technical progress}

In consultation with Dr. Katherine Yuracko, an expert in life-cycle analysis at the Oak Ridge National Laboratory, Florida International University's Hemispheric Center for Environmental Technology (FIU-HCET) has formulated a strategy for developing a life-cycle cost analysis tool for RSM that will complement the life-cycle decision methodology developed by Dr. Yuracko. In addition to providing decision-makers with RSM disposal options, the integrated decision tool will provide a clear indication as to which option is the most cost-effective. Other key issues that are being addressed by the LCCA decision methodology are

- A clear and common understanding of the meaning of each factor that is taken into account in current LCCAs. Therefore, one of the tasks that FIU-HCET will undertake is to compile a taxonomy of the factors and their definitions.

- A more accurate means of estimating future costs of maintaining waste disposal sites including facility surveillance and maintenance. Most importantly, current methodologies do not account 
for potential future liability in the event of accidental occurrences such as exposure or leakage at these facilities.

- Disposal Facility Summaries and/or Waste Disposition Maps indicating quantities of RSM and possible TSD alternatives have been obtained for the Fernald Environmental Management Project, Hanford Operations Site Environmental Restoration Disposal Facility, Idaho National Environmental Engineering Laboratory, Nevada Test Site, Oak Ridge Reservation, Savannah River Site, Rocky Flats Environmental Test Site, and Los Alamos National Laboratory. Together these facilities have a current stockpile of over one million tons of RSM.

\section{Assessment of current status}

Due to delays in obtaining RSM inventory information from Sanford, Cohen \& Associates, Inc. and Industrial Ecology, Inc., both of whom conducted RSM inventories on behalf of the Nuclear Regulatory Commission and the Environmental Protection Agency, a comparative analysis and reconciliation of existing inventories have not been possible. Alternative arrangements have been made to obtain this information, and FIU-HCET will deploy additional resources to this project to minimize delays and ensure that subsequent critical deadlines are met. Milestones 2 and 3 will be completed as scheduled.

Once the data from existing RSM inventories have been analyzed and reconciled by FIU-HCET, the results will be reviewed with DOE-EM's National Center of Excellence for Metals Recycle (NMR) at Oak Ridge and correlated to NMR's current estimates in order to derive a realistic estimate of DOE's present and future RSM inventories.

\section{Plans for the next two months}

During the next two months, the following will be accomplished:

- FIU-HCET will complete the inventory of the quantities and characteristics of RSM currently stockpiled at DOE facilities as well as RSM expected to be generated from future D\&D activities. A report on the inventory will be submitted to DOE on March 1, 1999.

- FIU-HCET shall contact DOE waste site managers and other appropriate personnel to discuss this scope of work for assessment of life-cycle costs for disposal of RSM. FIU-HCET shall ascertain the level of cooperation expected from these managers in providing full information needed for the assessment and to develop LCCAs. A list of site managers surveyed indicating their willingness to participate will be submitted to DOE.

\section{FIU-HCET collaborator}

Robert Tucker, (305) 348-6181. 


\title{
Legacy Waste Disposition for the Oak Ridge Reservation
}

\author{
Project Number: HCET-1999-D044
}

\section{Project objectives}

Deactivation and decommissioning (D\&D) of the surplus facilities at the Oak Ridge Reservation (ORR) will result in millions of cubic meters of waste of varying degrees of hazard and toxicity, requiring treatment, storage, and disposal (TSD). A large portion of the waste consists of low-level, uncharacterized, heterogeneous mixed waste streams. Currently, disposition paths do not exist at ORR for much of the mixed low-level waste (MLLW), which has to be sent to commercial waste facilities for disposition.

There are over 60 storage facilities on the ORR where MLLW is stockpiled. It is the goal of the U.S. Department of Energy (DOE) to eliminate this stockpile of legacy MLLW by the year 2006.

Several options exist for the TSD of contaminated waste streams at the ORR. These include neutralization, separation, vitrification, volume reduction by incineration or evaporation, packaging and direct disposal, and decontamination for reuse/recycling. In disposing of waste, the key objectives of the DOE's waste management program include safety, pollution prevention, waste minimization, and resource recovery. A clear understanding of proven TSD alternatives (disposition paths) for particular waste streams is therefore critical to achieving waste management goals and objectives. By their very nature, MLLW streams could potentially require an infinite number of disposition options for characterization, treatment, storage, and disposal, which could prove to be prohibitively costly. The need exists for a systematic means of evaluating MLLW streams and selecting the most appropriate disposition path for each stream from a limited number of options. This would minimize the number of disposition processes that would have to be set up to characterize, treat, store, and dispose of MLLW streams and would reduce costs for waste management.

Under this subtask, Florida International University's Hemispheric Center for Environmental Technology (FIU-HCET) will

- Perform a series of technical reviews for the DOE to aid in determining TSD options for MLLW streams at the ORR and to support the DOE's goal of eliminating the MLLW inventory by the year 2006.

- Investigate feasible TSD options and technologies for legacy MLLW streams at the ORR for which no disposition paths currently exist. 


\section{Major milestones}

\begin{tabular}{|c|l|l|l|}
\hline Milestone No. & \multicolumn{1}{|c|}{ Milestone Description } & \multicolumn{1}{|c|}{ Completion Criteria } & \multicolumn{1}{c|}{ Status } \\
\hline D044-M1 & $\begin{array}{l}\text { Report on TSD options for residue } \\
\text { from MLLW metal feeds to the } \\
\text { TSCA incinerator }\end{array}$ & $\begin{array}{l}\text { A set of feasible disposition paths for } \\
\text { the MLLW metal feeds to the TSCA } \\
\text { incinerator. }\end{array}$ & $\begin{array}{l}\text { Scheduled for } \\
\text { completion } \\
4 / 30 / 99\end{array}$ \\
\hline D044-M2 & $\begin{array}{l}\text { Report on TSD options for } \\
\text { contaminated accelerator lead } \\
\text { shielding. }\end{array}$ & $\begin{array}{l}\text { At least 2 feasible options for } \\
\text { disposing of the contaminated lead } \\
\text { shielding. }\end{array}$ & $\begin{array}{l}\text { Scheduled to } \\
\text { begin } 2 / 99 .\end{array}$ \\
\hline D044-M3 & $\begin{array}{l}\text { Report on TSD options for } \\
\text { contaminated cadmium plates. }\end{array}$ & $\begin{array}{l}\text { At least 2 feasible options for } \\
\text { disposing of the contaminated } \\
\text { cadmium plates. }\end{array}$ & $\begin{array}{l}\text { Scheduled to } \\
\text { begin 3/99. }\end{array}$ \\
\hline D044-M4 & $\begin{array}{l}\text { Report on wastewater residue TSD } \\
\text { options at Y-12. }\end{array}$ & $\begin{array}{l}\text { A set of feasible disposition paths for } \\
\text { the Y-12 wastewater residues. }\end{array}$ & $\begin{array}{l}\text { Scheduled to } \\
\text { begin } 4 / 99\end{array}$ \\
\hline D044-M5 & $\begin{array}{l}\text { Report on performance of PM- } \\
\text { CEMs in meeting EPA monitoring } \\
\text { guidelines. }\end{array}$ & $\begin{array}{l}\text { A statistical correlation of the } \\
\text { performances of the two tested CEMs. }\end{array}$ & $\begin{array}{l}\text { Scheduled to } \\
\text { begin } 5 / 99 .\end{array}$ \\
\hline D044-M6 & $\begin{array}{l}\text { Final report summarizing research } \\
\text { findings and disposal maps for } \\
\text { various MLLW streams. }\end{array}$ & $\begin{array}{l}\text { Final report submitted to Bechtel } \\
\text { Jacobs, Inc. }\end{array}$ & Due 10/31/99. \\
\hline
\end{tabular}

\section{Significant events}

- FIU-HCET is currently working with a waste management team led by Mr. Chuck Estes at Bechtel Jacobs, LLC, to assess and optimize the current process for characterizing, transporting, treating, and disposing of stored quantities of legacy waste at ORR, specifically 17,000 drums of MLLW for which disposition paths need to be developed. Due to an urgent need to finalize a Broad Spectrum Treatment Plan for the drums of MLLW, Mr. Estes was obliged to use the services of characterization contractors to define approximately 40 feasible waste disposition options for the MLLW inventory. This is a preliminary plan based on the experiences of the contractors and does not take account of the actual characteristics of the MLLW inventory. This has, to some extent, preempted but not negated much of the work to be performed by FIU-HCET under this task that was slated to commence in February 1999. On January 22, FIU-HCET met with Mr. Estes to clarify and redefine FIU-HCET's role in completing this task.

- To ensure that project deliverables and schedules are met, FIU-HCET will be fielding a full-time employee in February 1999 to work in Oak Ridge with Bechtel Jacobs to facilitate day-to-day contact and communication.

\section{Accomplishments and technical progress}

Based on discussions and a meeting with Mr. Estes, FIU-HCET has identified the following issues that will be addressed in completing its objectives for this task:

- There are approximately 17,000 drums of MLLW for which FIU-HCET will assist Bechtel Jacobs, Inc., to develop disposition paths. 
- Most of the waste in these drums can be characterized based on the accompanying Requests for Disposal (RFDs), of which there are approximately 10,000.

- A large portion of the waste was characterized before waste acceptance criteria (WAC) were developed for the waste disposal sites where they are to be dispositioned.

- Based on existing RFDs, FIU-HCET will perform an assessment of the MLLW inventory to assess whether the Broad Spectrum Treatment Plan developed by Bechtel Jacobs, Inc. is applicable to the inventory. If not, FIU-HCET will make recommendations for amending the Plan, including definition/redefinition of disposition options and paths.

\section{Assessment of current status}

With the deployment of a full-time project manager to Oak Ridge, FIU-HCET expects to maintain this project on schedule. This on-site presence will facilitate coordination of efforts between FIUHCET and Bechtel Jacobs, LLC, and promote rapid response to identified project needs and changes.

\section{Plans for the next two months}

The scope of FIU-HCET's effort on this subtask is summarized below and scheduled for completion by March 31, 1999. FIU-HCET will assess the MLLW Broad Spectrum Treatment Plan developed by Bechtel Jacobs, LLC, and conduct an engineering study of the current TSD process for MLLW. FIU-HCET will

- Obtain from Bechtel Jacobs a sample of 500 RFDs.

- Work with Bechtel Jacobs to assess and document the current process and options for characterizing, transporting/handling, treating, and disposing of the sample MLLW streams.

- Review the MLLW Broad Spectrum Treatment Plan and assess whether the plan adequately addresses all the paths necessary to disposition the sample waste streams. This will also involve reviewing external factors such as WACs and Department of Transportation regulations which may affect the logical grouping of drums into a particular path.

- Develop an optimized disposition process and options for MLLW.

\section{FIU-HCET collaborator}

Robert Tucker, (305) 348-6181 


\section{TANK FOCUS AREA (TFA)}

\section{MONTHLY PROGRESS REPORT}

FIU Principal Investigator

FIU TFA Program Manager

Focus Area Technical Leads

Program Officers
M.A. Ebadian

F. Mao

Kurt Gerdes

William Holtzscheiter

Peter Gibbons

John Wengle

Karl-Heinz Frohne

http://www.hcet.fiu.edu 


\title{
Waste Conditioning for Tank Slurry Transfer
}

\author{
Project Number: HCET-1998-T004
}

\section{Project objectives}

There are millions of gallons of radioactive waste slurries stored in underground tanks located at different U.S. Department of Energy (DOE)'s sites. DOE needs information and technologies to treat the wastes and close the tanks. Treatment of these wastes into safe waste forms and closure of these tanks require information of chemical and physical properties of the waste and fundamental data related to tank slurry conditioning, mixing, transport, and processing.

Florida International University's Hemispheric Center for Environmental Technology (FIU-HCET) is conducting research and examination on waste conditioning for tank slurry transfer. In this project, FIU-HCET is performing experimental tests to obtain reliable data in order to understand problems encountered in tank slurry mixing and transfer processes. Based on the data and results obtained from the experiments, FIU-HCET is investigating possible solutions to prevent pipeline plugging during slurry transfer and the problems that occur in slurry mixing. Additionally, this project has reviewed and compared the actual slurry natures at different DOE sites and facilities, such as Fluor Daniel Fernald (FDF), Oak Ridge National Laboratory (ORNL), Savannah River Sites (SRS), and Hanford, and identified the requirements for slurry transfer.

This project should accomplish the following:

- Determine the effect of chemical and physical properties on the tank slurry transfer process.

- Provide information for the transfer equipment design and operation.

- Identify and evaluate the most sensitive parameters that influence the waste conditioning and transfer operations.

\section{Major milestones}

\begin{tabular}{|c|l|l|l|}
\hline $\begin{array}{c}\text { Milestone } \\
\text { No. }\end{array}$ & \multicolumn{1}{|c|}{ Task Description } & \multicolumn{1}{|c|}{ Completion Criteria } & \multicolumn{1}{|c|}{ Status } \\
\hline T004-M1 & $\begin{array}{l}\text { Review previous work and adjust } \\
\text { experimental setup }\end{array}$ & $\begin{array}{l}\text { Experimental facility must fulfill } \\
\text { M2-6 test requirements }\end{array}$ & $\begin{array}{l}\text { On Schedule. } \\
\text { Due date: } \\
02 / 26 / 99\end{array}$ \\
\hline T004-M2 & $\begin{array}{l}\text { Measure surrogates particle size } \\
\text { distribution }\end{array}$ & $\begin{array}{l}\text { Perform six different particle size } \\
\text { distribution tests as described in } \\
\text { Table I in the PTP }\end{array}$ & $\begin{array}{l}\text { Due date: } \\
04 / 12 / 99\end{array}$ \\
\hline T004-M3 & Determine surrogates particle shapes & $\begin{array}{l}\text { Perform six particle geometry tests as } \\
\text { described in Table 2 in the PTP }\end{array}$ & $\begin{array}{l}\text { Due date: } \\
05 / 17 / 99\end{array}$ \\
\hline T004-M4 & $\begin{array}{l}\text { Characterize solid particle solubility } \\
\text { and crystallization behavior }\end{array}$ & $\begin{array}{l}\text { Perform nine solubility and } \\
\text { crystallization tests as described in } \\
\text { Table 3 in the PTP }\end{array}$ & $\begin{array}{l}\text { Due date: } \\
07 / 05 / 99\end{array}$ \\
\hline T004-M5 & Measure particle settling velocity. & $\begin{array}{l}\text { Perform 27 settling tests as described } \\
\text { in Table 4 in the PTP }\end{array}$ & $\begin{array}{l}\text { Due date: } \\
08 / 15 / 99\end{array}$ \\
\hline
\end{tabular}




\begin{tabular}{|c|l|l|l|}
\hline $\begin{array}{c}\text { Milestone } \\
\text { No. }\end{array}$ & \multicolumn{1}{|c|}{ Task Description } & \multicolumn{1}{|c|}{ Completion Criteria } & \multicolumn{1}{|c|}{ Status } \\
\hline T004-M6 & Measure slurry viscosity & $\begin{array}{l}\text { Perform 26 viscosity tests as } \\
\text { described in Table 5 in the PTP }\end{array}$ & $\begin{array}{l}\text { Due date: } \\
09 / 30 / 99\end{array}$ \\
\hline T004-M7 & $\begin{array}{l}\text { Perform data correlation and } \\
\text { documentation }\end{array}$ & Write a project final report & $\begin{array}{l}\text { Due date: } \\
10 / 30 / 99\end{array}$ \\
\hline
\end{tabular}

\section{Significant events}

- Slurry physical and chemical property measurements at different conditions were continued. The intent was to determine the effect of these conditions on the slurries properties.

- The waste conditioning experimental facility was adjusted to comply with the slurry test requirements as described in the project technical plans (PTP) for fiscal year 99 (FY99).

\section{Accomplishments and technical progress}

- This month's main activity was to continue the slurries physical and chemical properties analyses started last month. This included viscosity measurements at various conditions of $\mathrm{pH}$, temperature, and concentration.

- The experimental measurements and data correlation with the available data show that the slurry viscosity has slight variation at different temperatures. The graph in Figure 1 is an example of this trend.

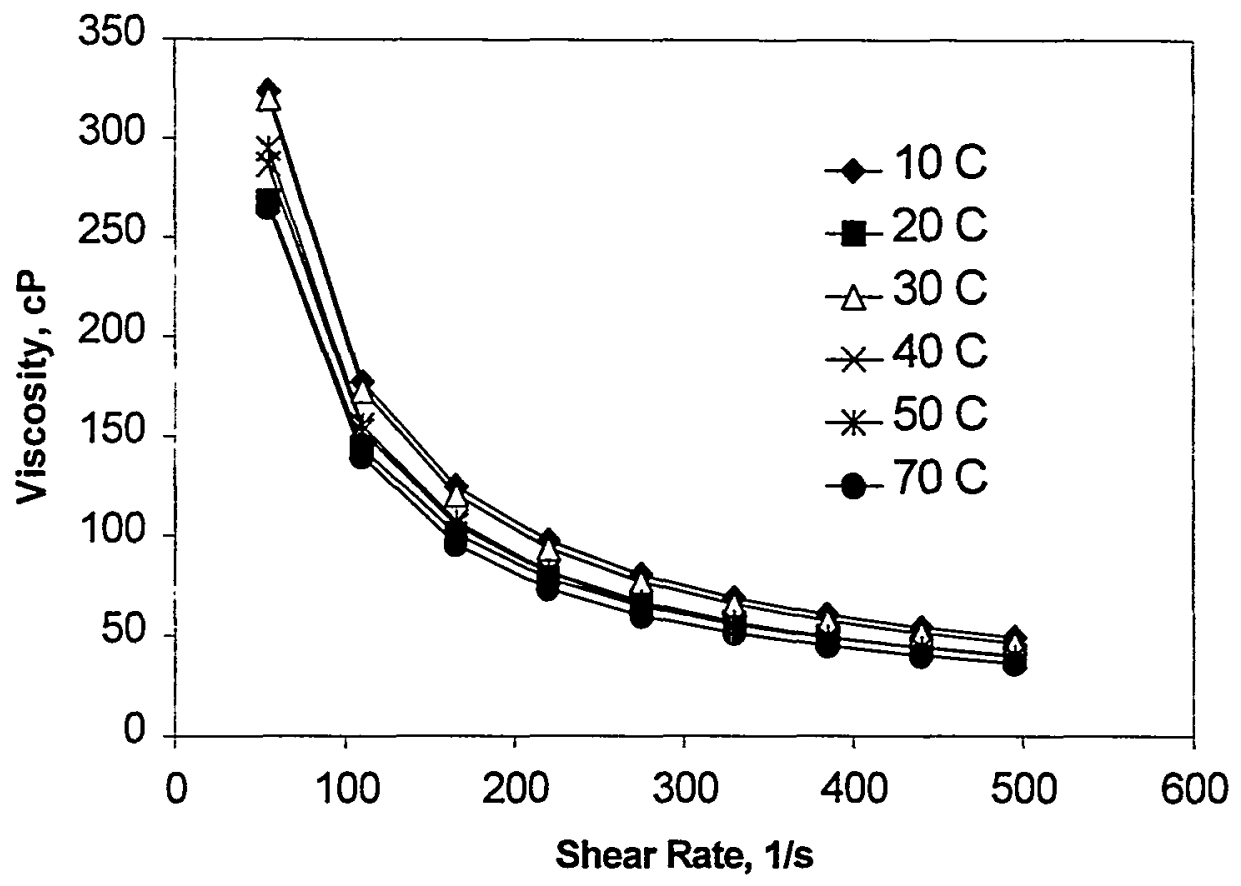

Figure 1. Effect of Temperature on SRS Slurry Viscosity (Conc. 19.7\%). 
- Furthermore, the graph in Figure 2 shows how the slurry $\mathrm{pH}$ affects its viscosity. As can be observed, the viscosity of the slurry is higher at higher $\mathrm{pH}$.



Figure 2. Effect of pH on SRS Slurry Viscosity (Conc. $19.7 \%$, Temp. $23^{\circ} \mathrm{C}$ ).

- In addition, slurry samples were prepared for particle size distribution analysis. This task consists of particle size distribution analysis in the settled portion as in the supernate layer, at different $\mathrm{pH}$.

- Also, a decision was made to analyze slurry samples corresponding to the plugging-andunplugging project. In this form, all samples from both projects will be analyzed under the same conditions for data comparison.

\section{Assessment of current status}

The project is on schedule, as described in the project milestones in the PTP. The physical and chemical property analyses performed at different conditions are critical to draw conclusions for the improvement of the waste conditioning process.

\section{Plan for the next two months}

- Continue with physical and chemical property measurements of available slurry samples at different concentrations.

- Check if particle settling rate affects viscosity under all conditions. If some settling occurs during viscosity measurements, it may lead to inconsistent results.

- Perform slurry particle size distribution analyses under different conditions. 
- Work together with the Plugging and Unplugging of Waste Transfer Pipelines (HCET-1998T005) project to compare results and improve testing methods.

\section{FIU-HCET collaborators}

Fuhe Mao, (305) 348-1838

Rubén Darío López, (305) 348-1872 


\title{
Plugging and Unplugging of Waste Transfer Pipelines
}

\author{
Project Number: HCET-1998-T005
}

\section{Project objectives}

As the waste tank clean-out and decommissioning program becomes active at the DOE sites, there is an increasing potential that the waste slurry transfer lines will become plugged and unable to transport from one tank to another or from the mixing tank to processing facilities. Whereas some sites, such as Savannah River, Hanford, and Oak Ridge, have already experienced plugged or blocked lines, plugging may occur at additional sites at the onset of waste transfer.

Florida International University's Hemispheric Center for Environmental Technology (FIU-HCET) will continue to investigate pipe plugging and unplugging behaviors of waste slurry transfer lines for a high-level waste ( $\mathrm{HLW}$ ) system on the waste transfer simulation flow loop in FY99. In addition to the pipe plugging caused by settling, pipe plugging and unplugging phenomena induced by gelling will also be studied by both experimental and theoretical methods. Some key aspects of particle deposition associated with pipe plugging will be addressed. These will include particle agglomeration leading to larger particles that fall out of suspension and particle deposition in the pipe at the end of the transfer as a function of pipe slope or dip depth. The experimental setup used for settling-induced plugging will be modified for the study of gelling-induced plugging and unplugging. The core-annular flow technology, which may be used to unplug the gel-caused blockage, will be examined.

In FY99, activities of industrial equipment tests and demonstrations of plug locating and pipe unplugging technologies will be coordinated by FIU-HCET, Numatec Hanford Corporation (NHC), Pacific Northwest National Laboratory (PNNL), Federal Energy Technology Center (FETC), and DOE sites. FIU-HCET will complete the design and construct the Plug Locating and Removal Demonstration test bed for the industrial equipment test and demonstration to be conducted in FY00. FIU-HCET will also plan additions to the large-scale (full-size) test bed required for pipeline inspection tools testing in the future.

The objectives of this work include the following:

- Further understand the pipeline plugging and unplugging mechanism by particle settling and gel formation.

- Identify and test industrial methods to locate and remove waste transfer pipeline blockage.

- Inspect and verify the condition of those pipelines. 


\section{Major milestones}

\begin{tabular}{|c|c|c|c|}
\hline Milestone No. & Milestone Description & Completion Criteria & Status \\
\hline T005-M1 & $\begin{array}{l}\text { Issue test plan for flow loop } \\
\text { pipeline plugging and unplugging } \\
\text { experiments }\end{array}$ & $\begin{array}{l}\text { Planned experimental studies of particle } \\
\text { settling caused plugging and unplugging } \\
\text { in the flow loop of both horizontal and } \\
\text { dipped pipes and planned activities of } \\
\text { gelation studies. }\end{array}$ & $\begin{array}{l}\text { Under revision. } \\
\text { Due by } 02 / 15 / 99\end{array}$ \\
\hline T005-M2 & $\begin{array}{l}\text { Experimental investigation of } \\
\text { settling-caused pipe plugging }\end{array}$ & $\begin{array}{l}\text { Reasonable data on slurry flow critical } \\
\text { velocity, resuspension velocity, plugging } \\
\text { and unplugging behaviors in a flow loop. }\end{array}$ & $\begin{array}{l}\text { Scheduled } \\
\text { completion } \\
03 / 30 / 99\end{array}$ \\
\hline $\mathrm{T} 005-\mathrm{M3}$ & $\begin{array}{l}\text { Theoretical study of settling- } \\
\text { caused plugging process }\end{array}$ & $\begin{array}{l}\text { Detailed flow field and particle } \\
\text { distribution of slurry flow in pipelines. }\end{array}$ & $\begin{array}{l}\text { Scheduled } \\
\text { completion } \\
05 / 28 / 99\end{array}$ \\
\hline T005-M4 & $\begin{array}{l}\text { Identify and prepare sludge } \\
\text { simulants and lubricating liquid } \\
\text { for gelling tests }\end{array}$ & $\begin{array}{l}\text { Sludge is capable of forming gel and } \\
\text { lubricant is immiscible in the gel. }\end{array}$ & $\begin{array}{l}\text { Scheduled } \\
\text { completion } \\
04 / 30 / 99\end{array}$ \\
\hline T005-M5 & $\begin{array}{l}\text { Design and modification of } \\
\text { experimental setup for gelling } \\
\text { tests }\end{array}$ & $\begin{array}{l}\text { Heating and cooling test sections will be } \\
\text { added to the existing flow loop to study } \\
\text { gelling process. }\end{array}$ & $\begin{array}{l}\text { Scheduled } \\
\text { completion } \\
05 / 28 / 99\end{array}$ \\
\hline T005-M6 & $\begin{array}{l}\text { Experimental investigation of } \\
\text { gelling-induced plugging and } \\
\text { unplugging }\end{array}$ & $\begin{array}{l}\text { Reasonable data of gelling process, } \\
\text { gelling-caused plugging and unplugging } \\
\text { behavior in pipelines. }\end{array}$ & $\begin{array}{l}\text { Scheduled } \\
\text { completion } \\
09 / 15 / 99 .\end{array}$ \\
\hline T005-M7 & $\begin{array}{l}\text { Issue report on results of flow } \\
\text { loop experiments of pipeline } \\
\text { plugging and unplugging by } \\
\text { slurry settling and gelling }\end{array}$ & $\begin{array}{l}\text { Report important experimental data to } \\
\text { help prevent plugging, and to unplug the } \\
\text { blocked pipeline when plugging occurs. }\end{array}$ & $\begin{array}{l}\text { Scheduled } \\
\text { completion } \\
09 / 30 / 99 .\end{array}$ \\
\hline T005-M8 & $\begin{array}{l}\text { Identify and determine industry } \\
\text { companies and potential } \\
\text { technologies for equipment tests } \\
\text { and demonstrations }\end{array}$ & $\begin{array}{l}\text { List of companies and technologies with } \\
\text { contact information. }\end{array}$ & $\begin{array}{l}\text { Scheduled } \\
\text { completion } \\
11 / 01 / 99 .\end{array}$ \\
\hline T005-M9 & $\begin{array}{l}\text { Design of the test bed for } \\
\text { equipment tests and } \\
\text { demonstrations }\end{array}$ & $\begin{array}{l}\text { Detailed design drawings of the } \\
\text { pipelines for every case in the test bed. }\end{array}$ & $\begin{array}{l}\text { Scheduled } \\
\text { completion } \\
03 / 30 / 99\end{array}$ \\
\hline T005-M10 & $\begin{array}{l}\text { Construction of the test bed for } \\
\text { equipment tests and } \\
\text { demonstrations }\end{array}$ & $\begin{array}{l}\text { All the cases of pipelines will be } \\
\text { fabricated with the specified material } \\
\text { and dimensions. }\end{array}$ & $\begin{array}{l}\text { Scheduled } \\
\text { completion } \\
09 / 15 / 99 .\end{array}$ \\
\hline T005-M11 & $\begin{array}{l}\text { Plan addition to large-scale test } \\
\text { bed for pipeline inspection }\end{array}$ & $\begin{array}{l}\text { Documentation of modification of the } \\
\text { existing test bed for pipeline inspection. }\end{array}$ & $\begin{array}{l}\text { Scheduled } \\
\text { completion } \\
07 / 30 / 99 .\end{array}$ \\
\hline T005-M12 & $\begin{array}{l}\text { Draft and distribute the year-end } \\
\text { report of the plugging and } \\
\text { unplugging project }\end{array}$ & $\begin{array}{l}\text { Report cover detailed experimental } \\
\text { studies and progress of the full-size test } \\
\text { bed. }\end{array}$ & $\begin{array}{l}\text { Scheduled } \\
\text { completion } \\
11 / 15 / 99 \text {. }\end{array}$ \\
\hline
\end{tabular}

Note: The project technical plan for FY99 is currently being revised. Therefore, the milestones are subject to change in the following months.

\section{Significant events}

- The year-end report of FY98 of the project has been revised and finalized according to the comments and suggestions from Dr. Fadel F. Erian at PNNL. 
- Based on the suggestions for the experimental setup, the positive displacement pump will be kept in the flow loop. However, the pump will be replaced to incorporate a higher flow rate in order to cover the experimental data within high flow velocity range.

- The new drawing on the flow loop, incorporating an additional differential pressure transducer and the new sampling tubes, is finished and will be reviewed by Peter Gibbons at NHC and Dr. Fadel F. Erian at PNNL.

- The fittings for installing the new differential pressure transducers have been ordered. Some other related preparations for the flow loop modification are in progress. Once the flow loop modification plan is approved by NHC and PNNL, the flow loop will be modified.

- The simulated case \#3 for the Large-Scale Plugging/Unplugging Demonstration Test Bed is revised based on discussion with Dr. Fadel Erian at PNNL.

\section{Accomplishments and technical progress}

\section{Part 1--Flow Loop Research on Pipeline Plugging and Unplugging}

Figure 1 shows the major part of the modified flow loop, in which the pump, the slurry mixing tank, and the collection tank are not shown. Compared with the original flow loop, an additional differential pressure transducer is to be installed in the developing section of the flow loop (just after the pump). The objective is to verify the difference in pressure drops between the developing section and the developed section of the flow loop. The location of the additional differential pressure transducer is also illustrated in Figure 1. The test section was divided into Sections $\mathrm{A}$ through $\mathrm{E}$ for the purpose of construction.

Figure 2 shows the new design of the sampling tubes that will be installed in the horizontal pipe. In FY98, FIU-HCET used the simple sampling tube of 0.25 -inch diameter with vertical to the main flow direction. When the slurry sample is drained through the sampling tube, the flow field is affected by the draining, and this introduced error. In the new design, the sampling tube is bent 90 degrees so that the sampling tube is parallel to the main flow direction. The distance between the sampling tube and the inside pipe wall surface is only $0.16 \mathrm{inch}$. One entire sampling tube arrangement consists of a pair of sampling tubes, with the axial distance of nearly 4 inches. One is for the top point, and the other is for the bottom point. The detailed sampling tube geometry is illustrated in Figure 3. The new sampling system will be discussed with Dr. Fadel Erian from PNNL.

In FY98, the maximum flow velocity achieved in the current horizontal pipe loop is $1.2 \mathrm{~m} / \mathrm{s}$. In some cases, such value was found not enough to cover the flow range required to obtain reasonable critical velocity. Therefore, based on the suggestions from Dr. Fadel F. Erain at PNNL, a new slurry pump, with the same type of positive displacement but with higher flow rate, is to be used in the flow loop. The expected flow rate is $25 \mathrm{GPM}$, and the pressure is $50 \mathrm{psi}$. The flow rate range is consistent with what Dr. Fadel F. Erain suggested, $4000 \mathrm{~L} / \mathrm{h}$, with equivalent flow velocity of $3.0 \mathrm{~m} / \mathrm{s}$ in the horizontal pipe. The preparation of such a pump is in process.

After the modification plan is approved by NHC and PNNL, the existing flow loop will be modified. 


\section{Part 2 Large-Scale Industrial Equipment Test Bed of Plug Locating and Unplugging}

Technologies

\subsection{Introduction}

Florida International University's Hemispheric Center for Environmental Technology (FIU-HCET) has been designing and will construct a Large-scale Industrial Equipment Test Bed of Plug Locating and Unplugging Technologies. The concept design of the test bed has been performed and continuously revised in the past months. Three revised simulated cases have been produced from the five original cases, based on the discussions with Peter Gibbons of Numatec Hanford Corporation (NHC), Dr. Fadel F. Erian at PNNL, and the document "Functions and Requirements for Blockage Locating and Removal Methods in Waste Transfer Lines," which was provided by NHC.

In FY99, the designs of the Large-scale Test Bed will be completed, followed by the construction and demonstration of the test bed and its equipment at FIU-HCET.

The concept design of the large-scale test bed for the simulated case \#1(original case \#1 and \#5), and simulated case \#2 (original case \#2, \#3, and \#4) are included in the December FY98 monthly report.

\subsection{New Design of Pipeline}

The latest revision of the simulated case \#3 of the full-size test bed is provided in this monthly report. The revision was performed based on the discussion with Peter Gibbons of NHC and Dr. Fadel F. Erian at PNNL.

Figure 4 shows the revised buried pipe. It consists of two pipes with 3- and 4-inch diameters, respectively, which are buried in a trapezoidal-shaped mixture of clay and sand with $95 \%$ compaction. The more detailed dimensions of the design are to be determined.

For simulated case \#1 and simulated case \#2, the feasibility of using Lexan as the material is assessed under the guidance of Dr. Fadel F. Erian of PNNL. Its transparent quality would be a great asset in detecting the location of and/or removing the blockage in the future.

\section{Assessment of current status}

The FIU-HCET team and Dr. Erian all agreed that the pump capacity of the flow loop should be increased. The replacement of the slurry pump is in process.

- Another differential pressure transducer is being added in the developing section of the flow loop. Test cases at additional volume concentrations for SRS or Hanford slurry simulants will be carried out as long as the flow loop is modified.

- The progress of the large-scale demonstration test bed for plugging and unplugging will depend to some extent on FETC's Progress Call for Technologies of the Plugging Inspections and the Unplugging Tools.

\section{Plans for the next two months}

- The flow loop will be modified. Test cases at additional volume concentrations of slurry simulants in horizontal pipelines will be performed after the modification. 
- The detailed design of the large-scale industrial test bed of plug locating and unplugging technologies will be further reviewed and finalized.

- The cost analysis for the different pipeline material including Lexan wil be conducted for the full-size test bed.

- The composition of the slurry simulants to be used in the large-scale industrial test bed will be studied and identified.

- The FY99 PTP will be revised based on the current progress and technical needs.

- Issue revised test plan for flow loop by $2 / 15 / 99$.

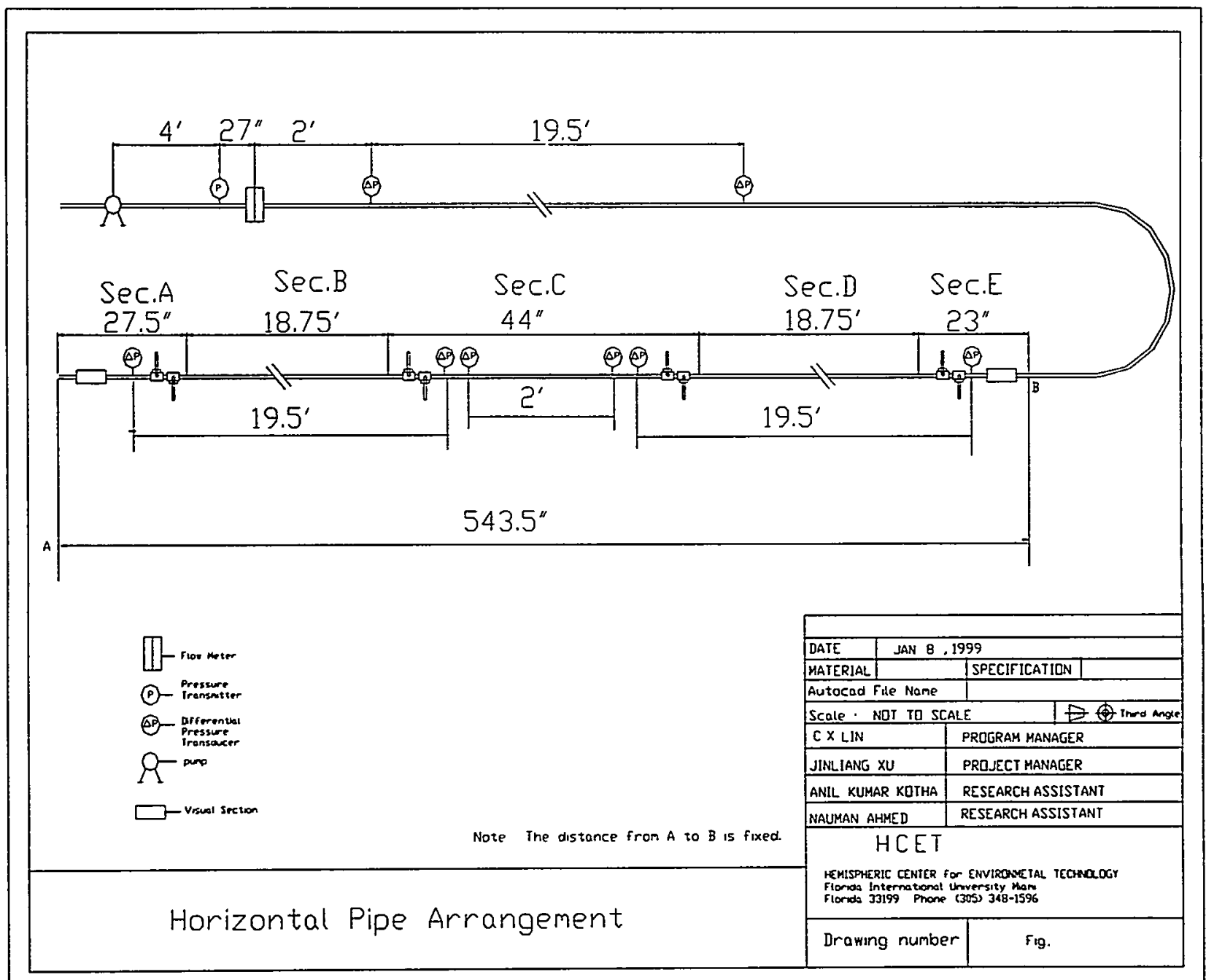

Figure 1. The modified flow loop, including one additional differential pressure transducer, and the modified sampling tubes. 


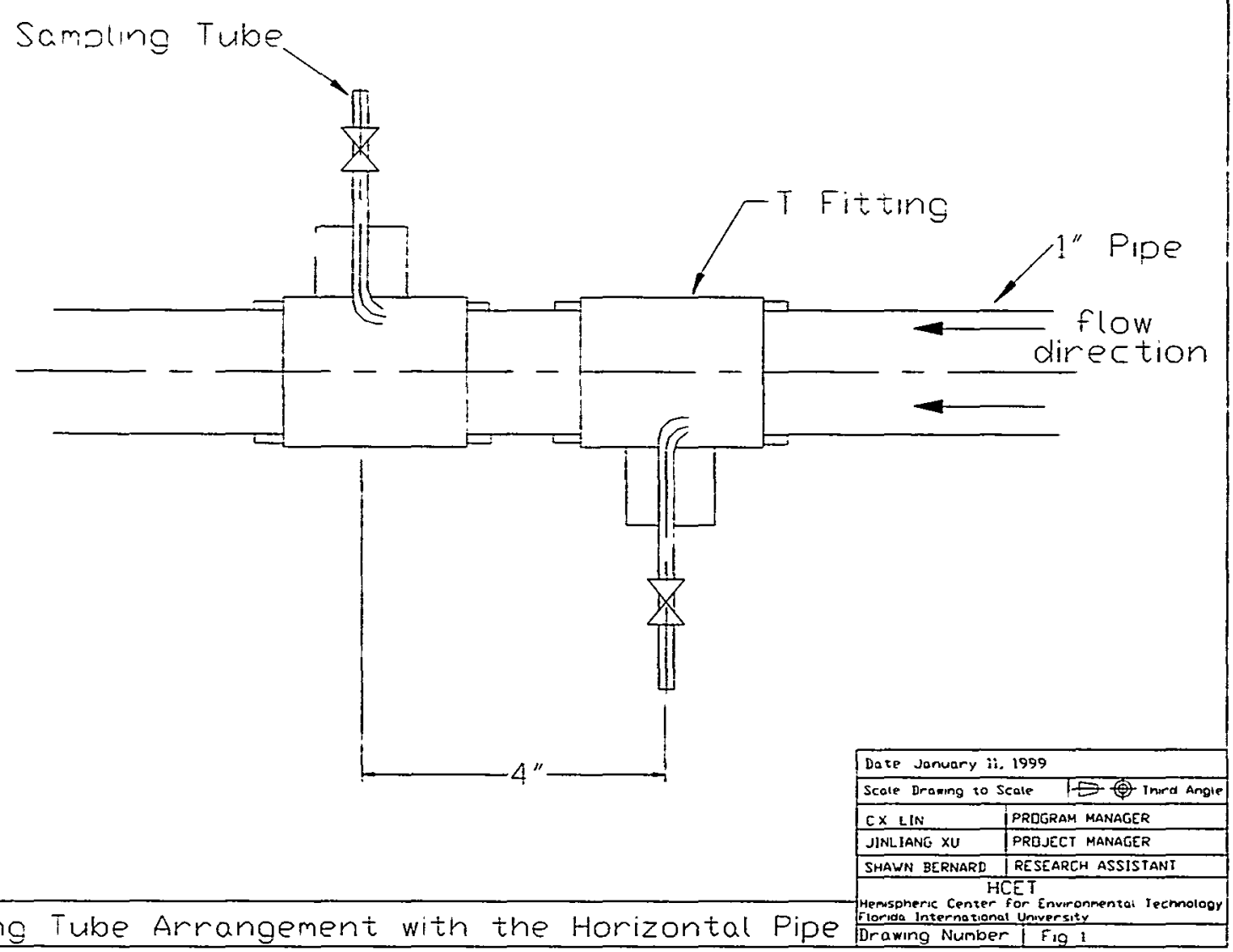

Figure 2. Sampling tube arrangement with the horizontal pipe. 


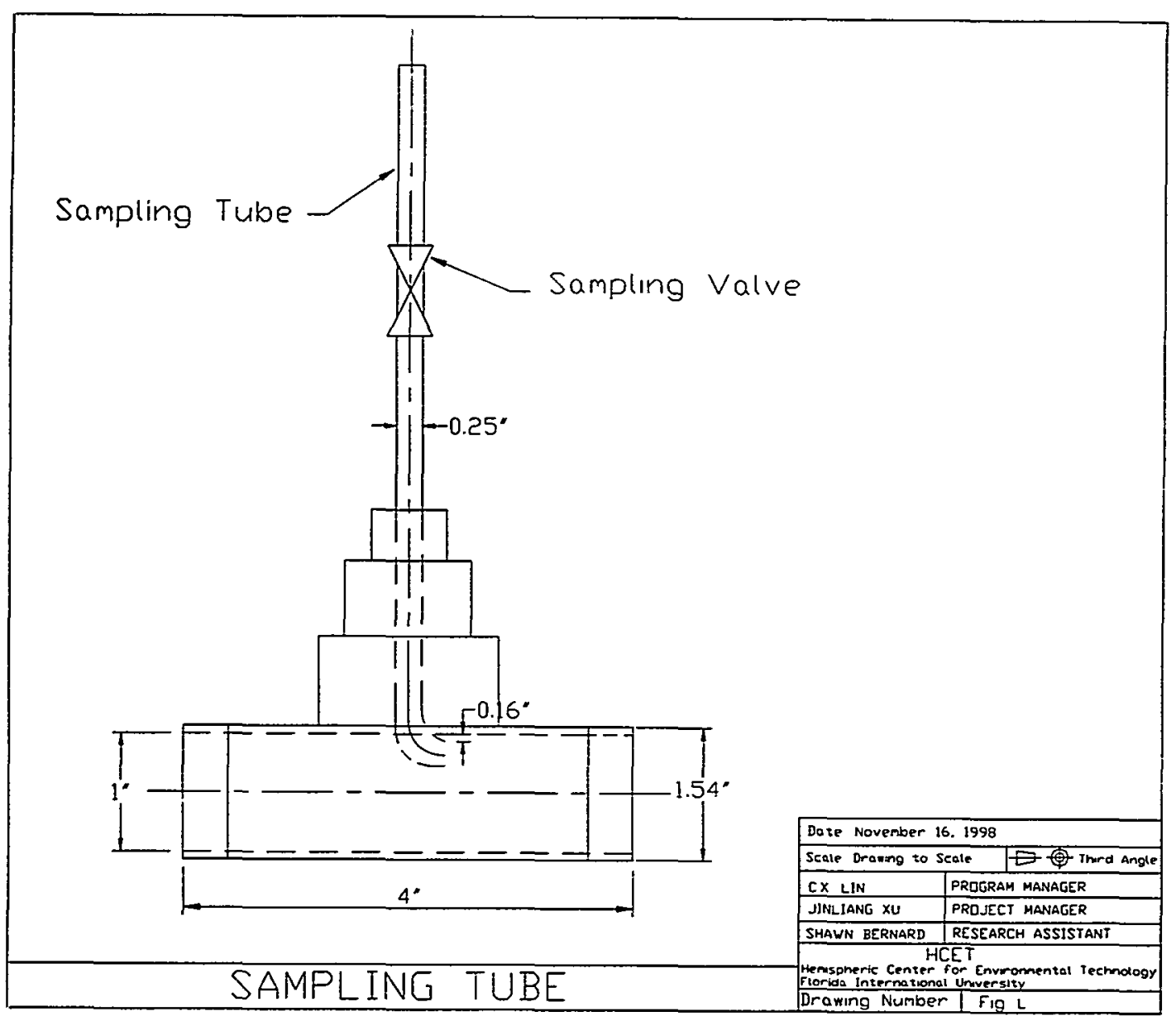

Figure 3. Detailed sampling tube geometry. 


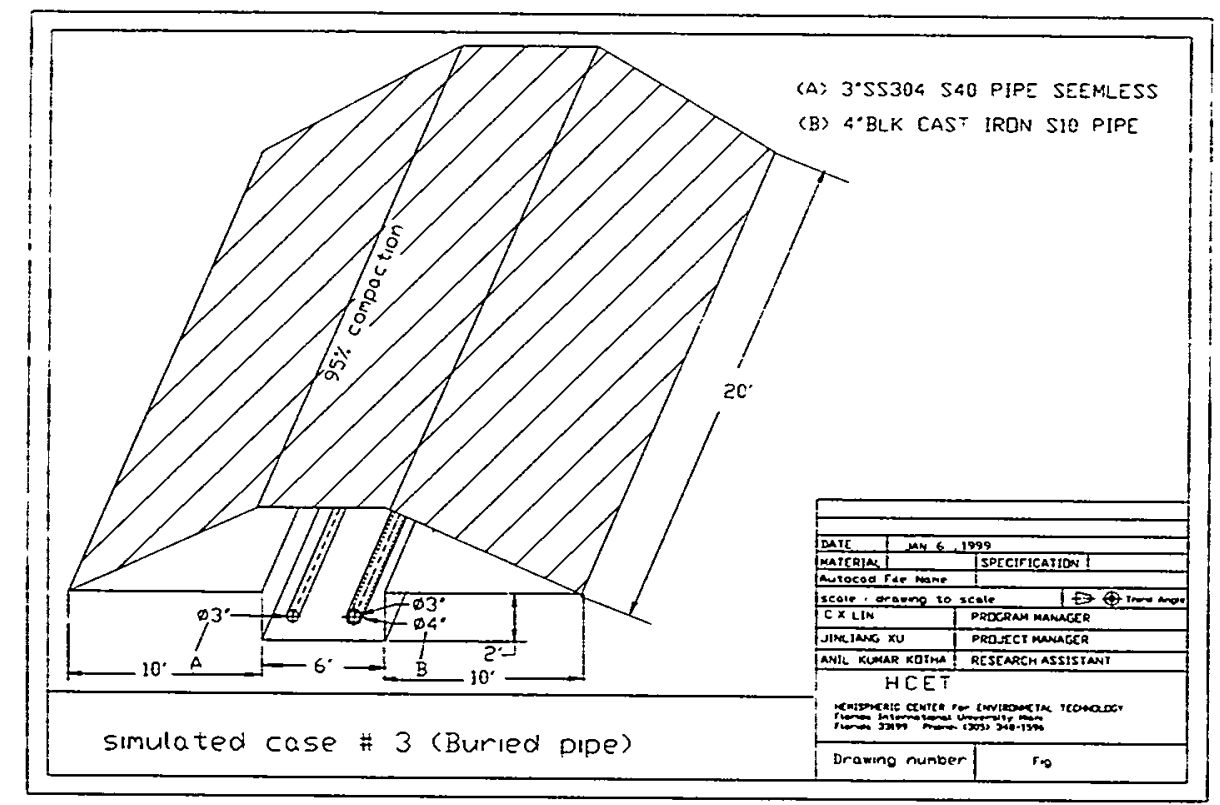

Figure 4. Pipeline plugging and unplugging large-scale demonstration (buried pipe). Simulated case \#3.

\section{FIU collaborators}

C. X. Lin, (305) 348-1596

J. L. Xu, (305) 348-6732 


\title{
Investigation of Waste Glass Pouring Process Over a Knife Edge
}

\author{
Project Number: HCET-1997-T003
}

\section{Project objectives}

Vitrification is the process of capturing radioactive waste in glass. The Savannah River Site's (SRS) Defense Waste Processing Facility (DWPF) is one of the facilities using the vitrification technology to treat and immobilize radioactive waste since March 1996. However, the operation has been marked by extreme difficulty in maintaining a stable pouring process. There have been flow fluctuations accompanied by an unusual flow phenomenon, termed "wicking". In this situation, the falling glass stream wavers and departs from a normal vertical trajectory. The pour spout and associated hardware connecting it to the canister have been coated and often plugged with glass. The objective of the project is to investigate the pouring behavior of molten glass over a pour spout knife edge.

The work to be performed at Florida International University's Hemispheric Center for Environmental Technology (FIU-HCET) in support of the Tank Focus Area (TFA) Technology Implementation Manager (EM-50) and the Savannah River Technology Center (SRTC) consists of three phases. Phase 1 involved the assembly, construction, and testing of a melter capable of supplying molten glass at operational flow rates over a break-off point knife edge. Phase 2 evaluated the effect of glass and pour spout temperatures as well as glass flow rates on the glass flow behavior over the knife-edge. Phase 3 (current phase) of the project will identify the effects on wicking that result from varying the knife edge diameter and height as well as changes to the back-cut angle of the knife edge.

\section{Major milestones}

\begin{tabular}{|c|l|l|l|}
\hline Milestone \# & \multicolumn{1}{|c|}{ Task Description } & \multicolumn{1}{|c|}{ Completion Criteria } & \\
\hline T003-M1 & Installation of an additional heat zone & $\begin{array}{l}\text { Achievement of } 1150^{\circ} \mathrm{C} \text { by the } \\
\text { knife edge }\end{array}$ & $\begin{array}{l}\text { On schedule } \\
\text { DUE DATE: } 1 / 31 / 99\end{array}$ \\
\hline T003-M2 & Report the effect of glass chemistry & $\begin{array}{l}\text { Experiments II-SF-1, II-SF-2, II-SF- } \\
\text { 3, II-SF-4, II-SF-5, II-TF-1, and II- } \\
\text { TF-2 }\end{array}$ & DUE DATE: $3 / 31 / 99$ \\
\hline T003-M3 & $\begin{array}{l}\text { Report the effect of crud deposits on } \\
\text { the back side of the knife edge }\end{array}$ & $\begin{array}{l}\text { Experiments ES-1, ES-2, ES-3, and } \\
\text { ES-4 }\end{array}$ & DUE DATE: $4 / 30 / 99$ \\
\hline T003-M4 & $\begin{array}{l}\text { Report the effect of eroded knife } \\
\text { edges }\end{array}$ & $\begin{array}{l}\text { Experiments ES-5, ES-6, ES-7, ES- } \\
\text { 8, ES-9, ES-10, ES-11, ES-12, ES- } \\
\text { 13, ES-14, ES-15, and ES-16 }\end{array}$ & DUE DATE: $9 / 30 / 99$ \\
\hline T003-M5 & Final report & $\begin{array}{l}\text { Draft and distribute the Final report } \\
\text { on results of the project delivered to } \\
\text { DOE }\end{array}$ & Due Date: $10 / 31 / 99$ \\
\hline
\end{tabular}




\section{Significant events}

- A final copy of the project year-end report was printed for publishing and client distribution. This report corresponds to activities performed throughout fiscal year 1998 (FY98).

- In order to test the effect of pouring spout geometry on the glass pouring behavior, four technical drawings (Figures 1-4) for the eroded knife edge experiments have been completed and forwarded to the SRTC for review. These will be forwarded to the machine shop once approved by SRTC.

\section{Accomplishments and technical progress}

- During baseline runs, heat losses at the end of the pour spout resulted in temperatures less than $1100^{\circ} \mathrm{C}$. An additional heat zone has been installed on the pour spout to account for end heat loss. Thermal profiling for the new and improved pour spout will be carried out during the next month.

- The glass feeder section of the melter has been redesigned. The feeder section now consists of a 3 -inch gate valve that will be used for feeding crushed glass. This was done to replace the feeder flange that was leaking $\mathrm{N}_{2}$ gas at high temperatures $\left(>1000^{\circ} \mathrm{C}\right)$.

- The technical drawings (Figures 1-4) for the eroded knife edge experiments have been completed and forwarded to the SRTC for review. These will be forwarded to the machine shop once approved by SRTC. Eroded knife edge experiments will be carried out to simulate the actual eroded knife-edge at a DWPF melter.

- Mr. Sharad Sharma has joined the glass pouring project as of January 4, 1999. He has a mechanical engineering background and has a master's degree from Florida International University. Mr. Sharma comes highly recommended. During his tenure as a graduate student, he was involved with other Tank Focus Area (TFA) projects at FIU-HCET.

\section{Assessment of current status}

The project is on schedule. As per the PTP for FY99, the modifications to the melter/furnace need to be completed by end of January 1999 as indicated by milestone T003-M1.

\section{Plans for the next two months}

The plans for the next period include the following:

- Assembly of the melter and installation inside the furnace.

- SRS has been contacted for delivery of a "new" glass shipment for task 2 as shown in Table 2. Task 2 will focus on the effect of glass chemistry on pouring behavior that may be caused by different surface tension and viscosity properties. The nominal pour spout geometry will be used. Table 1 shows the steady state and pulsating flow experimental runs planned for task 2 . 
Table 1. Proposed glass pouring tests for Task 2

\begin{tabular}{|c|c|c|c|}
\hline $\begin{array}{l}\text { Test } \\
\text { Number }\end{array}$ & $\begin{array}{l}\text { Glass Temp. } \\
{ }^{0} \mathrm{C}\end{array}$ & $\begin{array}{l}\text { Pour Spout } \\
\text { Temp. } \\
{ }^{0} \mathrm{C}\end{array}$ & $\begin{array}{l}\text { Glass Flow } \\
\mathrm{lb} / \mathrm{hr}\end{array}$ \\
\hline II-SF-1 & 1050 & 1050 & 75 \\
\hline II-SF-2 & 1050 & 1050 & 200 \\
\hline II-SF-3 & 1050 & 1050 & 450 \\
\hline II-SF-4 & 950 & 1050 & 200 \\
\hline II-SF-5 & 1150 & 950 & 200 \\
\hline
\end{tabular}

Table 2. Proposed glass pouring tests for Task 2

\begin{tabular}{lllll}
\hline $\begin{array}{l}\text { Test } \\
\text { Number }\end{array}$ & $\begin{array}{l}\text { Glass /Spout } \\
\text { Temp } \\
{ }^{0} \mathrm{C}\end{array}$ & $\begin{array}{l}\text { Initial Steady } \\
\text { State Flow } \\
\text { lbs/hr }\end{array}$ & $\begin{array}{l}\text { Oscillating Flow } \\
\text { Range } \\
\text { lbs/hr. }\end{array}$ & Pour Spout Configuration \\
\hline II-TF-1 & $1050 / 1050$ & 100 & $50-150$ & Original Knife Edge \\
II-TF-2 & $1050 / 1050$ & 250 & $150-350$ & Original Knife Edge \\
\hline
\end{tabular}

\section{FIU-HCET collaborators}

Rajiv Srivastava, (305) 348-6621

Yaozhong Liu, (305) 348-1824 



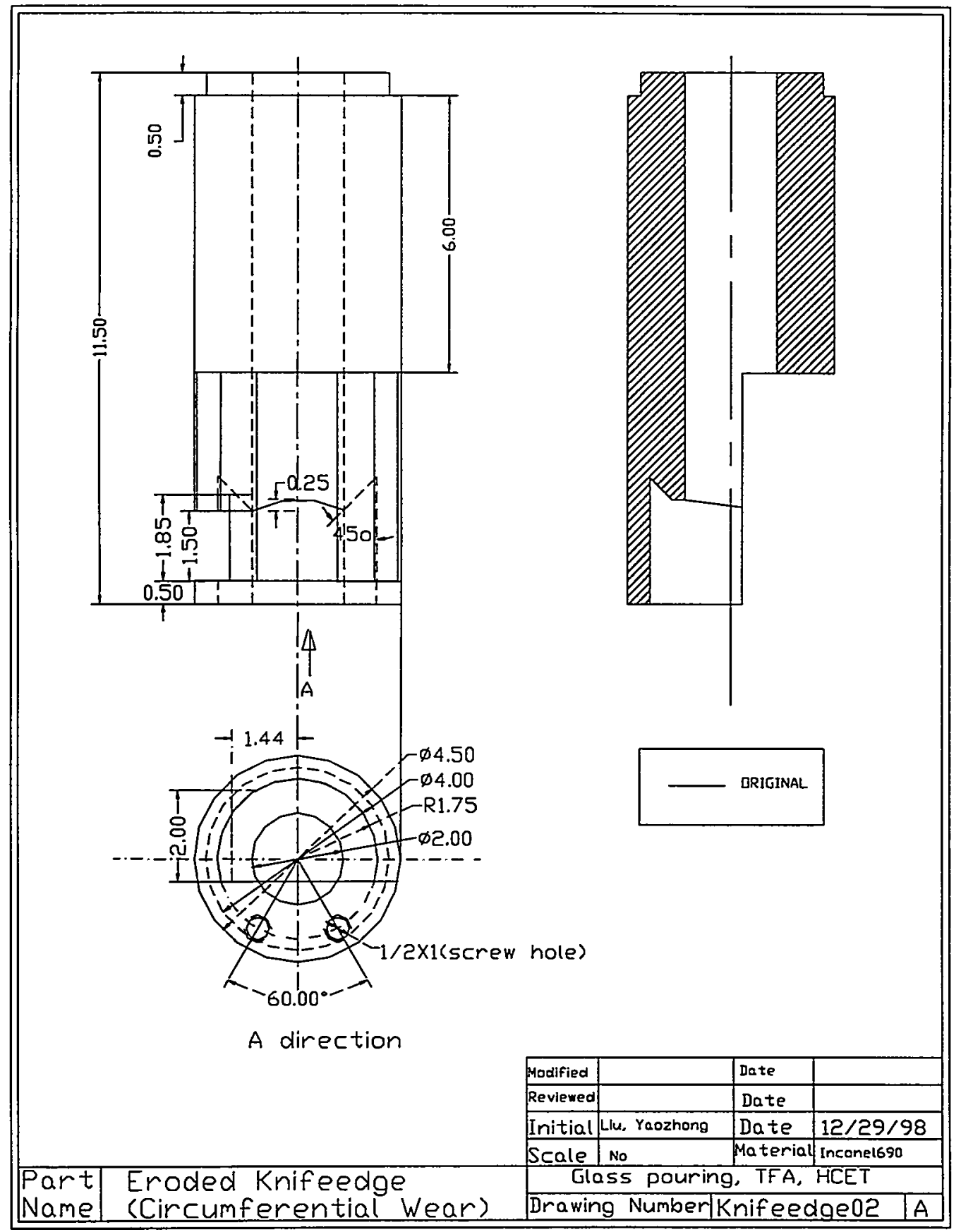

Figure 1. Eroded knife-edge for simulating cicumferential wear glass pouring experiments. 


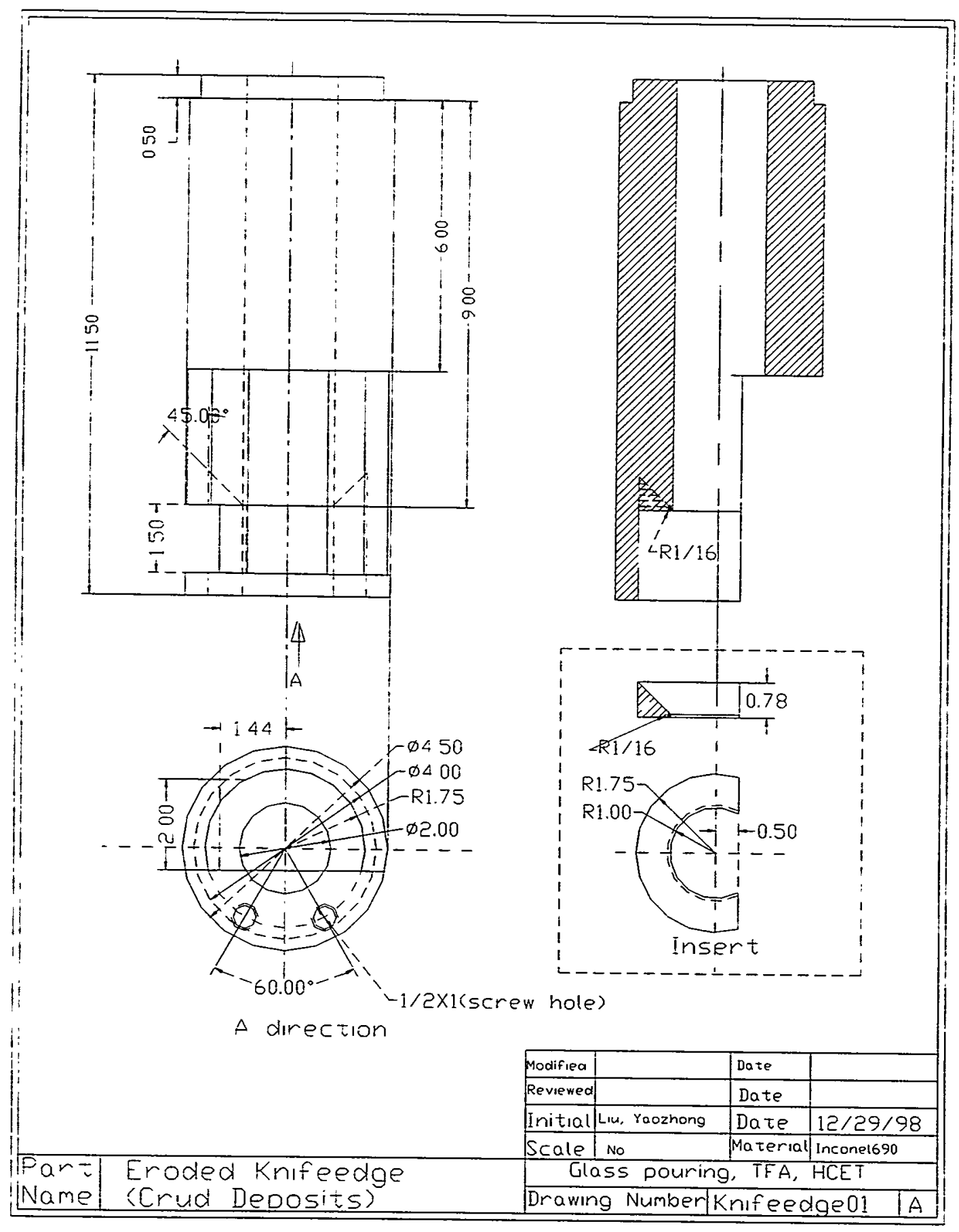

Figure 2. Eroded knife-edge for simulating knife-edge with crud deposits, glass pouring experiments. 


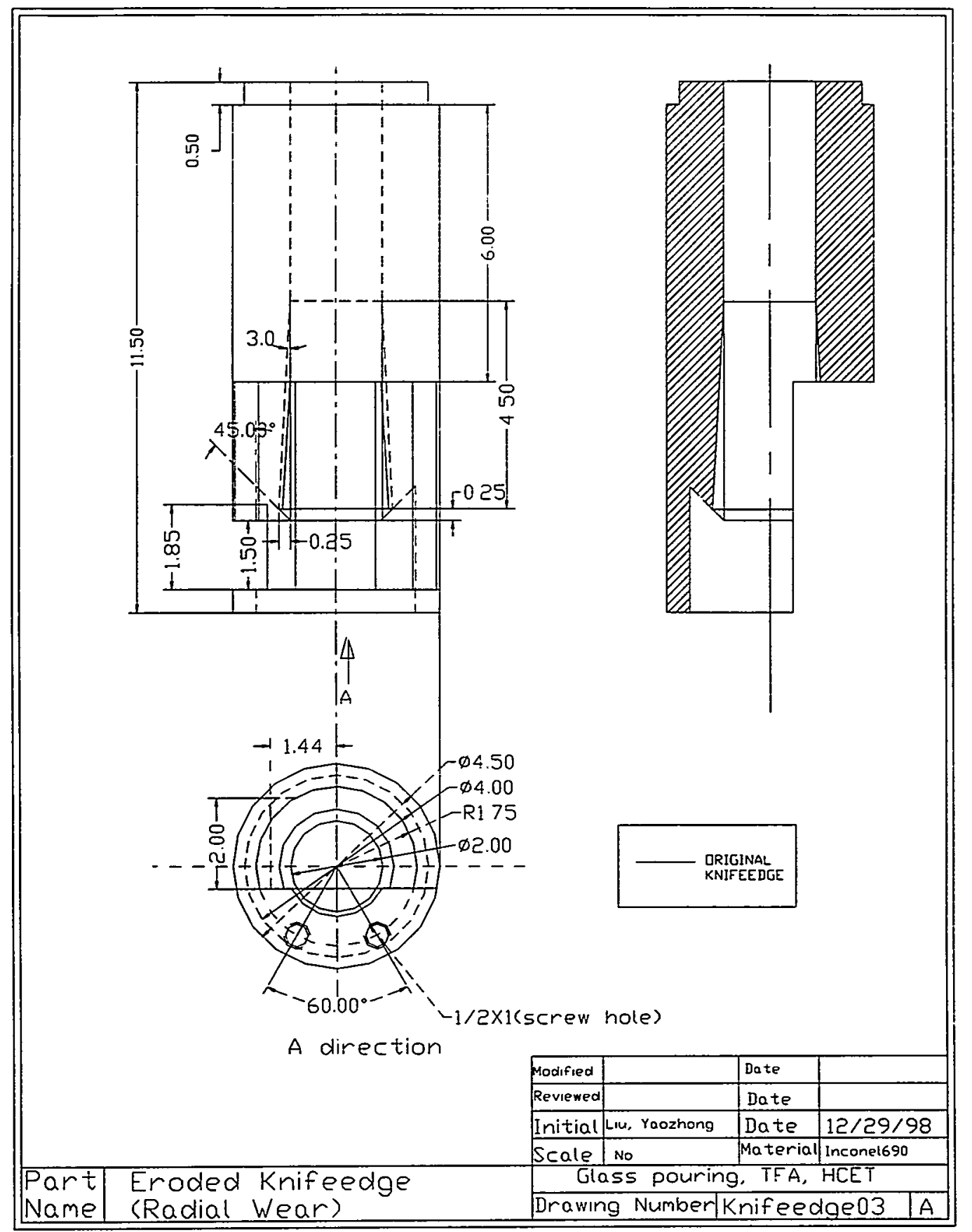

Figure 3. Eroded knife-edge for simulating radial wear glass pouring experiments. 


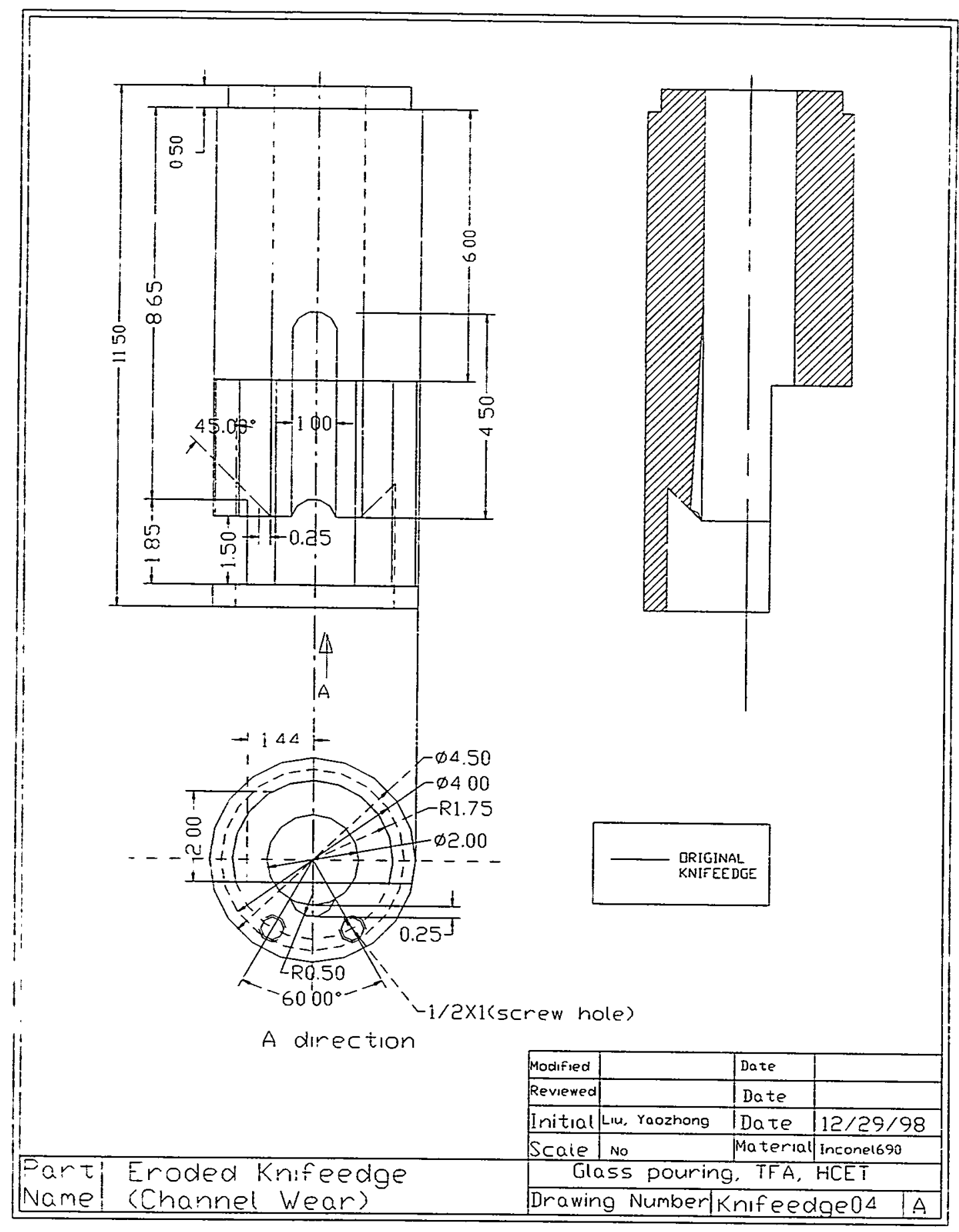

Figure 4. Eroded knife-edge for simulating channel wear glass pouring experiments. 


\section{CHARACTERIZATION, MONITORING, AND SENSOR TECHNOLOGY}

MONTHLY PROGRESS REPORT

FIU Principal Investigator FIU CMST Program Manager Focus Area Technical Lead Program Officers
M.A. Ebadian

Paul Szerszen

Charles Nalezny

John Wengle

Karl-Heinz Frohne

http://www.hcet.fiu.edu 


\section{Online Measurement of the Progress of Decontamination}

Project Number: HCET-1998-C005

\section{Project objectives}

The accurate characterization of contaminants is a critical task during several different phases of deactivation and decommissioning (D\&D) operations. This project focuses directly on in-process characterization. Present characterization technologies typically require the cessation of decontamination activities, while the contamination remaining is assessed. This usually requires the decontamination activity to cease awaiting a separate radiological survey.

The specific aims of this subtask include the following:

- Find in-process characterization methods, especially in the area of radiation sensor systems that can be integrated with a suitable decontamination technology in order to combine decontamination and characterization activities.

- Include in the technology integration data collection, storage, and transmission components on the instrument for remote monitoring and computer downloading functions, allowing for continuous decontamination activities coupled by real-time assessments of the amount of contamination remaining. The result would be an overall gain in productivity accompanied by cost and time savings. A second important advantage would be that a minimum amount of material could be removed with a commensurate minimum production of residual waste.

- Adapt an existing decontamination technology with commercially available characterization technologies to develop a prototype instrument that will be assessed and then commercially deployed. A closed-system decontamination technology will be selected that utilizes a vacuum or contaminant collection system and will be integrated with appropriate radiation sensing devices and data collection components. This integration of technologies will yield an improved instrument that may be continuously operated, removing contaminated materials and simultaneously assessing the removal progress.

\section{Major milestones}

\begin{tabular}{|c|l|l|l|}
\hline Milestone No. & \multicolumn{1}{|c|}{ Milestone Description } & \multicolumn{1}{|c|}{ Completion Criteria } & \multicolumn{1}{|c|}{ Status } \\
\hline C005-M1 & Operational prototype & Prototype functional & $\begin{array}{l}\text { Due 3/3/99 } \\
\text { *Delayed until } \\
\text { 4/15/99 }\end{array}$ \\
\hline C005-M2 & Demonstration at FIU-HCET & Documented demonstration of prototype & $\begin{array}{l}\text { Due 5/30/99 } \\
\text { *Delayed until } \\
\end{array}$ \\
& & & $6 / 30 / 99$ \\
\hline C005-M3 & Deployment & Initiate deployment at DOE site & Due 8/28/99 \\
\hline C005-M4 & Year end report & Submission & Due $9 / 30 / 99$ \\
\hline
\end{tabular}

*As per FY99 PTP and Assessment of Current Status following, a design and implementation review required revisions to design and procurement. Milestone 1 is expected to be delayed until no later than April 15, 1999, and milestone 2 until no later than June 30, 1999. 


\section{Significant events}

Following design review, new sensors have been identified for integration.

\section{Accomplishments and technical progress}

- Alternative technologies and vendors for sensors identified.

- Operator interface and associated components design refined.

\section{Assessment of current status}

Review of preliminary system design and proposed implementation revealed discrepancies between design and ordered equipment with original scope and plan. A revised design and requisition is in process with expected minimal disruption of the initial milestone and task procession. Parallel projects are providing synergism and effectively accelerating the rate of progress. One of these projects, High Productivity Vacuum Blasting System, includes real-time operator feedback of the efficacy of the decontamination process. Another is Integrated Vertical and Overhead Decontamination and includes real-time characterization of vertical and overhead surfaces during decontamination. The function and implementation of the three sensor systems will by intent include interchangeable processes and components. The primary decontamination machine for the instant project prototype is available at FIU-HCET.

\section{Plans for the next two months}

- Purchase components for radiological sensing, operator interface, data logging, and spatial orientation.

- Generate revised design drawings for component integration.

- Initiate integration of components into prototype assembly.

- Continue negotiations for DOE facility deployment.

- Continue development of an FIU-HCET demonstration plan.

\section{FIU collaborator}

Richard Musgrove, (305) 348-6622 


\section{Remote Surveillance of Facilities Awaiting \\ Deactivation and Decommissioning}

Project Number: HCET-1998-C006

\section{Project objectives}

FY99 is the second year of the three-year project. Many United States Department of Energy (DOE) sites -- Albuquerque Operations Office, Chicago Operations Office, Idaho Operations Office, Ohio Operations Office, Oak Ridge Operations Office, and Savannah Operations Office -- require remote surveillance of their facilities such as production areas, structures, utilities, equipment, drums, tanks, and effluent lines. Currently, these facilities awaiting deactivation and decommissioning (D\&D) must be periodically surveyed for various criteria including contamination levels, structural deterioration, water intrusion, animal intrusion, integrity of storage containers, the atmospheric conditions, and radioactive and hazardous substance releases. The surveys themselves are intrusive, time-consuming, expensive, and expose survey personnel to radioactive contamination and radiation. The purpose of this project is to develop a remote surveillance system that is capable of collecting data from a DOE site (remote station) and transmitting the data to a central location (base station).

Following are the objectives of the project:

- Define specific surveillance needs among the facilities awaiting D\&D.

- Select appropriate sensors for different facilities and test them for their performance.

- Select components of the measuring system, integrate them, and test the performance of the sensors and the system.

- Select appropriate data collection, storage, transmission, and receiving units.

- Design a central monitoring unit.

- Integrate the different units into a prototype surveillance system and test the system.

- Test the system at a DOE site.

- Deploy the system at a DOE site.

- Design and implement a plan for commercialization. 
Major milestones

\begin{tabular}{|c|c|c|c|}
\hline Milestone No. & Milestone Description & Completion Criteria & Status \\
\hline \multicolumn{4}{|l|}{ FY98 } \\
\hline $\mathrm{C} 006-\mathrm{M}_{1}$ & Decision on facility selection & Deployment facility selected. & $\begin{array}{l}\text { Due: } 12 / 1 / 97 \\
\text { Outstanding. }\end{array}$ \\
\hline $\mathrm{C} 006-\mathrm{M} 2$ & $\begin{array}{l}\text { Consulting survey personnel } \\
\text { and regulators }\end{array}$ & Report submitted. & $\begin{array}{l}\text { Due: } 1 / 1598 . \\
\text { Outstanding. }\end{array}$ \\
\hline $\mathrm{C} 006-\mathrm{M} 3$ & $\begin{array}{l}\text { Evaluation of available } \\
\text { technologies }\end{array}$ & Survey w/life cycle cost analysis. & $\begin{array}{l}\text { Due: } 3 / 15 / 98 \\
\text { Survey begun. }\end{array}$ \\
\hline$\overline{\mathrm{C} 006-\mathrm{M} 4}$ & Components testing & Procurement and test. & $\begin{array}{l}\text { Due: } 8 / 1 / 98 \\
\text { Outstanding. }\end{array}$ \\
\hline C006-M 5 & System integration & Integrated and tested. & $\begin{array}{l}\text { Due: } 10 / 1 / 98 \\
\text { Outstanding. }\end{array}$ \\
\hline C006-M 6 & Year end report & Report completion. & $\begin{array}{l}\text { Due: } 10 / 30 / 98 \\
\text { Complete. }\end{array}$ \\
\hline \multicolumn{4}{|l|}{ FY99 } \\
\hline $\mathrm{C} 006-\mathrm{M} 1$ & Testing at FIU-HCET & $\begin{array}{l}\text { Components and integrated unit } \\
\text { tested at FIU-HCET }\end{array}$ & $\begin{array}{l}\text { Due: } 1 / 11 / 99 . \\
\text { Purchase of } \\
\text { components delayed } \\
\text { pending a firm DOE } \\
\text { site commitment. }\end{array}$ \\
\hline $\mathrm{C} 006-\mathrm{M} 2$ & Testing at a DOE site & $\begin{array}{l}\text { Tested at a DOE site for site-specific } \\
\text { parameters }\end{array}$ & Due: $4 / 16 / 99$ \\
\hline $\mathrm{C} 006-\mathrm{M} 3$ & System Improvement & Modifications completed. & Due: $5 / 17 / 99$ \\
\hline C006-M4 & Performance evaluation & $\begin{array}{l}\text { Performance evaluated under } \\
\text { ambient environmental conditions }\end{array}$ & Due: $8 / 27 / 99$ \\
\hline C006-M5 & Deployment plan & DOE site deployment plan created. & Due: $10 / 1 / 99$ \\
\hline $\mathrm{C} 006-\mathrm{M} 6$ & Commercialization plan & $\begin{array}{l}\text { Industrial partner interested in } \\
\text { commercialization of the system } \\
\text { identified }\end{array}$ & Due: $10 / 30 / 99$ \\
\hline $\mathrm{C} 006-\mathrm{M} 7$ & Year-end report & Report completion & Due: $11 / 30 / 99$ \\
\hline
\end{tabular}

\section{Significant events}

Projects run over boundaries of fiscal years. Milestones not met do not need to be completed next fiscal year (FY99). A system to track last year's milestones was devised and is shown in the milestone table above.

\section{Accomplishments and technical progress}

- Project review was conducted. New management was selected.

- Design review was initiated.

- Fernald POC, Keith Wilkerson, was contacted regarding confirmation of intent to participate in deployment activities. 


\section{Assessment of current status}

Project is currently under review and assessment of status and progress to date. Revisions as to scope, schedule, and deliverables will be made.

\section{Plans for the next two months}

- Obtain a firm commitment from a site.

- Purchase system components, assemble, and test at Florida International University's Hemispheric Center for Environmental Technology (FIU-HCET).

\section{FIU collaborator}

Richard Musgrove, (305) 348-6622 


\title{
Measurement of Alpha Contamination on Contaminated \\ Surfaces Using an Electret Ion Chamber
}

\author{
Project Number: HCET-1998-C008
}

\section{Project objectives}

In and around nuclear plants such as vitrification plants, fuel reprocessing plants, uranium plants, thorium plants, waste storage facilities, reactors, and radiological laboratories, surfaces (floors, walls, ceiling, and equipment) and soil may become contaminated with alpha-emitting radionuclides such as uranium, thorium, radium, americium, or plutonium. It is important to be able to measure such contamination and classify it as below or above the permissible levels. The permissible levels of alpha contamination are low. The U. S. Department of Energy (DOE) requires low-cost, reliable methods for measuring low levels of alpha contamination. Current methods for measurement of low levels of alpha contamination in a large facility are expensive and expose survey personnel to radiation. The goal of this two-year project:

- Develop a system for low-cost and reliable measurement of surface alpha contamination and to deploy it at a DOE site. This involves the use of commercially available electret ion chambers and their calibration using reference alpha sources.

- Determine times required for measurement of an alpha contamination at the free release level for six different chamber-electret combinations, their useful range, effect of environmental radon and gamma radiation on alpha contamination measurement, cost comparison with baseline technologies, and demonstration and deployment at a site.

\section{Major milestones}

\begin{tabular}{|c|l|l|l|}
\hline Milestone No. & Milestone Description & \multicolumn{1}{|c|}{ Completion Criteria } & \multicolumn{1}{c|}{ Status } \\
\hline C008-M1 & Cost-benefit analysis & $\begin{array}{l}\text { Data showing performance of EIC vs. } \\
\text { baseline technologies }\end{array}$ & $\begin{array}{l}\text { Scheduled for completion } \\
\text { by 12/15/98. } \\
\text { Measurements using EICs } \\
\text { alpha probe and completed } \\
\text { at a test-bed at FIU-HCET. } \\
\text { Cost-comparison being } \\
\text { performed. Vendor } \\
\text { negotiations and site user } \\
\text { solicitation delayed } \\
\text { completion. }\end{array}$ \\
\hline C008-M2 & Deployment plan & $\begin{array}{l}\text { Commitment for use of EICs for alpha } \\
\text { contamination from one or more DOE } \\
\text { sites }\end{array}$ & $\begin{array}{l}\text { Scheduled for completion } \\
\text { by 2/8/99. Liaison at Oak } \\
\text { Ridge informed FIU- } \\
\text { HCET of a potential } \\
\text { deployment opportunity for } \\
\text { the EIC technology for } \\
\text { floor characterization at K- } \\
\text { 1420. Main source of } \\
\text { contamination: depleted } \\
\text { and enriched uranium. }\end{array}$ \\
\hline
\end{tabular}




\begin{tabular}{|c|l|l|l|}
\hline Milestone No. & Milestone Description & \multicolumn{1}{|c|}{ Completion Criteria } & \multicolumn{1}{c|}{ Status } \\
\hline C008-M3 & Deployment & $\begin{array}{l}\text { Deployment of the EIC system at one } \\
\text { or more DOE sites }\end{array}$ & $\begin{array}{l}\text { Scheduled for completion } \\
\text { by } 5 / 17 / 99\end{array}$ \\
\hline C008-M4 & Information flow & $\begin{array}{l}\text { Availability of procedures, } \\
\text { instructions, manuals, and information } \\
\text { on developments and improvements to } \\
\text { DOE }\end{array}$ & $\begin{array}{l}\text { Scheduled for completion } \\
\text { before } 10 / 30 / 99\end{array}$ \\
\hline
\end{tabular}

\section{Significant events}

- Calibration of the EICs for alpha contamination has been performed. Response factors, useful range, and effect of radon and gamma radiations on alpha contamination measurement have been determined.

- A paper entitled "Measurement of Surface Alpha Contamination Using Electret Ion Chamber" has been submitted for publication to the Health Physics Journal.

- A test-bed has been set up at Florida International University's Hemispheric Center for Environmental Technology (FIU-HCET). Measurements have been performed using EIC and alpha probe. Electrets with Mylar and Tyvek covers will be tested on the test-bed. The data generated in this experiment will be used to advocate utilization of bathroom tiles as test-bed for calibration of instruments. The experiments on tiles add the following value to the project:

- A comparison of the EICs with a baseline technology is made for alpha contamination on tiles.

- Tiles can have uniform contamination and can serve as test-beds for calibration of instruments.

- Different types of tiles can have large variation in activity

\section{Accomplishments and technical progress}

- The response of the $145-\mathrm{mL}$ windowless chamber for an ${ }^{241} \mathrm{Am}$ alpha source placed at different distances from the chamber's centerline was measured. Its response for other alpha emitters $\left({ }^{237} \mathrm{~Np},{ }^{239} \mathrm{Pu}\right.$, and $\left.{ }^{244} \mathrm{Cm}\right)$ placed in line with the chamber center and electrets of different thicknesses was also measured.

- The response of $960-\mathrm{mL}$ chambers, with and without aluminized Mylar windows, for different alpha sources placed at different distances from the chamber's centerline was measured.

- Interference of environmental radon and gamma radiation on alpha contamination measurement was determined for both $145-\mathrm{mL}$ and $960-\mathrm{mL}$. The minimum times required for measurement of alpha contamination, as well as useful ranges for different combinations of electrets and chambers, were determined. With about 10 percent measurement uncertainty, an alpha contamination at the level of free release $\left(1 \mathrm{dpm} \mathrm{cm} \mathrm{cor}^{-2}\right.$ for transuranics) can be measured with the $145-\mathrm{mL}$ chamber in $76,6.5$, or 2.7 hours depe ing upon the type of electret (LT, ST, or sensitive) used. Using the $960-\mathrm{mL}$ chamber and similar electrets, the same contamination can be measured in 19, 1.8, and 0.75 hours, respectively. The measurement times will be nearly double when a Mylar window is used for the chambers. A large number of EICs can be deployed 
simultaneously to measure surface contamination in any large facility. EICs are light-weight and can be easily placed on different surfaces, such as floors, walls, and ceilings for alpha contamination measurement. An electret can be used many times before its charge drops to a low value that necessitates its replacement with a new electret.

- A test-bed has been set up at FIU-HCET. Measurements have been performed using EIC and alpha probe.

\section{Assessment of current status}

- The system has been calibrated and is ready for demonstration. FIU-HCET is contacting representatives from Fernald, Oak Ridge, Rocky Flats, and Savannah River for demonstration of the technology. Among these sites, there is strong potential for demonstration and deployment at Oak Ridge (K-1420).

- Measurements performed on ceramic tiles in an apartment have demonstrated the usefulness of the system for measurement of low levels of alpha contamination (less than one-half the free release limit for transuranics).

- Rad Elec, Inc., vendor of EICs, tested electret ion chambers at a $12 \mathrm{~m} \times 12 \mathrm{~m}$ bay at the East Tennessee Technology Park (ETTP), Oak Ridge, during the early part of November 1998. The results show that an alpha contamination lower than free release level can be measured in one day and that a large number of EICs can be simultaneously deployed to perform low-costmeasurement in a large facility.

\section{Plans for the next two months}

- Analyzing the measurement performed at a test bed set up at FIU-HCET. These will provide a comparative study with a baseline technology-time required for measurement, cost of measurement, and radiation exposures likely to be received.

- Liaison at Oak Ridge informed FIU-HCET of a potential deployment opportunity for the EIC technology for floor characterization at K-1420. The main source of contamination is depleted and enriched uranium. FIU-HCET will be planning and performing a demonstration of the EIC at this facility. Measurement will also be performed using an alpha probe for cost comparison and performance evaluation.

- Submitting an abstract on measurements performed at a test-bed to Health Physics Society meeting.

\section{FIU-HCET collaborator}

S.K. Dua, (305) 348-1640 


\title{
Review of Current Characterization and Monitoring Practices at DOE Sites
}

\author{
Project Number: HCET-1999-C009
}

\section{Project objectives}

The goal of this project is to document current practices (baseline technology) for environmental technologies in the areas of site characterization and waste/processing monitoring at DOE sites. Data concerning each technology's cost and performance will be tabulated in a database. This information will assist the Characterization, Monitoring, and Sensor Technology Crosscutting Program (CMST$\mathrm{CP}$ ) in evaluating innovative technologies by facilitating the comparison of performance and cost data for the new technologies to the baseline technologies.

This activity, during its previous stages in FY97 and FY98, collected and compiled information from technology users, purchasers, and project sponsors. This information was published and converted into a database. FY99 is the first year that this project is managed by Florida International University's Hemispheric Center for Environmental Technology (FIU-HCET). FY99 activities include the following objectives:

- Review the current characterization and monitoring practices and baseline technologies at Hanford (RL) and Oak Ridge (OR).

- Collect and assess cost and performance data for these baseline technologies.

- Update the database to include this new information.

\section{Major milestones}

\begin{tabular}{|c|l|l|l|}
\hline Milestone No. & \multicolumn{1}{|c|}{ Description } & \multicolumn{1}{|c|}{ Completion Criteria } & \multicolumn{1}{c|}{ Status } \\
\hline C009-M1 & $\begin{array}{l}\text { Evaluate Current DOE } \\
\text { Characterization and } \\
\text { Monitoring Needs at } \\
\text { Hanford and Oak Ridge. }\end{array}$ & $\begin{array}{l}\text { Table of the current STCG needs indicating title, } \\
\text { description, requirements, regulations, baseline } \\
\text { method/technology, and point of contacts }\end{array}$ & $\begin{array}{l}\text { Completed on } \\
1 / 11 / 99, \text { before } \\
\text { due date of } \\
2 / 11 / 99\end{array}$ \\
\hline C009-M2 & $\begin{array}{l}\text { Identify DOE Baseline } \\
\text { Characterization and } \\
\text { Monitoring Technologies } \\
\text { at Hanford and Oak Ridge. }\end{array}$ & $\begin{array}{l}\text { List of the baseline methods and technologies } \\
\text { currently used to meet the STCG needs. }\end{array}$ & $\begin{array}{l}\text { On schedule to } \\
\text { completed by } \\
4 / 16 / 99\end{array}$ \\
\hline C009-M3 & $\begin{array}{l}\text { Describe the baseline } \\
\text { technologies and the DOE } \\
\text { requirements they meet. }\end{array}$ & $\begin{array}{l}\text { List of the description and performance data of } \\
\text { each method/technology identified in milestone } \\
\text { \#2. }\end{array}$ & $\begin{array}{l}\text { Scheduled to } \\
\text { begin } 4 / 16 / 99\end{array}$ \\
\hline C009-M4 & $\begin{array}{l}\text { Assess costs of use of } \\
\text { baseline technologies }\end{array}$ & $\begin{array}{l}\text { Table of the cost data of each method/technology } \\
\text { identified in milestone \#2. }\end{array}$ & $\begin{array}{l}\text { Scheduled to } \\
\text { begin } 5 / 17 / 99\end{array}$ \\
\hline C009-M5 & $\begin{array}{l}\text { Maintain and describe the } \\
\text { CMST-CP current practice } \\
\text { database }\end{array}$ & $\begin{array}{l}\text { Incorporation of the data from milestones \#1,2,3 } \\
\text { and 4 into a database }\end{array}$ & $\begin{array}{l}\text { To be } \\
\text { completed by } \\
10 / 30 / 99\end{array}$ \\
\hline C009-M6 & $\begin{array}{l}\text { Prepare year-end report for } \\
\text { FY99 }\end{array}$ & $\begin{array}{l}\text { Report summarizing the accomplishments of } \\
\text { Fiscal Year } 1999 \text { for this project. }\end{array}$ & $\begin{array}{l}\text { To be } \\
\text { completed by } \\
11 / 30 / 99\end{array}$ \\
\hline
\end{tabular}




\section{Significant events}

- The database has been transferred from Krell Institute to FIU-HCET.

- Milestone 1 was completed on 1/11/99. Milestone 1 is a table of current site technology coordinating group (STCG) needs indicating title, description, requirements, regulations, baseline method/technology, and points of contact.

\section{Accomplishments and technical progress}

- Completed the review and tabulation of the STCG needs for Oak Ridge and Hanford. The STCG needs are continually being upgraded by the STCG groups, and the current needs are posted on the STCG Internet site. The characterization and monitoring needs chosen are those that relate to the technical focuses of CMST-CP for FY99-01 identified in the CMST-CP Annual Performance Plan for Fiscal Year 1999.

- Continued the review and tabulation of the STCG baseline technologies currently used to realize these needs for Oak Ridge and Hanford.

- Initiated work on developing and designing the database. The tables of the STCG needs and the points of contact have been entered into the database.

\section{Assessment of current status}

This project is proceeding and no scheduling deadlines have been missed. Milestone 1 has been completed. Currently, no impediments are known that could delay the on-schedule completion of milestone 2.

\section{Plans for the next two months}

- Complete the review and tabulation of the baseline technologies currently used to meet the STCG needs for the Hanford site and Oak Ridge (milestone 2).

- Initiate the description of the baseline technologies (milestone 3).

- Initiate acquiring cost and performance data for the baseline technologies. Data will be obtained from end users, vendors, STCG members, and available documentation.

- Initiate contacting site personnel to arrange interviews and a site visit.

- Continue work on developing database.

\section{FIU collaborator}

Hans Weger, (305) 348-6620 


\title{
Demonstration and Deployment of CMST-CP Technologies
}

\author{
Project Number: HCET-1998-C010
}

\section{Project objectives}

The Characterization, Monitoring, and Sensor Technology Crosscutting Program (CMST-CP) exists to deliver appropriate characterization, monitoring, and sensor technologies to the Department of Energy (DOE), Office of Waste Management (EM-30), Office of Environmental Restoration (EM40), and Office of Facility Transition and Management (EM-60).

The purpose of this project is to assist CMST-CP with the final steps of this process. In short, it will help to take the technologies developed by CMST-CP to their ultimate use in the field. It is also a goal of this project to strengthen CMST-CP relationships with the users with the idea of deploying technologies more quickly and efficiently. To that end, Florida International University's Hemispheric Center for Environmental Technology (FIU-HCET) will help coordinate some of the deployment and related activities between the CMST-CP and the site users. In addition, this activity will directly support CMST-CP's D\&D coordinator.

To assist CMST-CP, FIU-HCET will provide the following:

- Examine the technology development activities and work together with CMST-CP to develop schedules for demonstration and deployment of these technologies.

- Match the technologies with characterization and monitoring needs of the customers.

- Choose sites to help facilitate demonstration and/or deployment.

- Use FIU-HCET's existing relationships with the rest of EM and the other focus areas to assist CMST-CP in selling the use of its technologies.

- Once an agreement has been reached, work with the customer to refine the demonstration/deployment process and schedule. If the user and CMST-CP so desire, FIUHCET could then coordinate and perform the demonstration at the user's site.

\section{Major milestones}

\begin{tabular}{|c|l|l|l|}
\hline Milestone No. & \multicolumn{1}{|c|}{ Milestone Description } & \multicolumn{1}{|c|}{ Completion Criteria } & \multicolumn{1}{c|}{ Status } \\
\hline C010-M1 & $\begin{array}{l}\text { Schedule \& number } \\
\text { demonstrations and/or } \\
\text { deployments }\end{array}$ & Definitive list of activities generated. & Due 12/15/98* \\
\hline C010-M2 & Choose sites & $\begin{array}{l}\text { Deployment/demonstration sites } \\
\text { identified. }\end{array}$ & Due 12/15/98* \\
\hline C010-M3 & Demonstrations & Complete scheduling and organization & Due 3/31/99 \\
\hline C010-M4 & Deployment & Fiscal year & Due 10/30/99 \\
\hline C010-M5 & Marketing & Fiscal year & Due 10/30/99 \\
\hline
\end{tabular}

* As per Assessment of current status below, revisions are in process to match new project scopes. 


\section{Significant events}

Due to funding cuts from CMST-CP, the scope of this project is being fundamentally changed.

\section{Accomplishments and technical progress}

Due to funding cuts from CMST-CP, the scope of this project is being fundamentally changed.

\section{Assessment of current status}

- Review of project scope revealed discrepancies between intended purpose and realizable accomplishments. A revised scope, tasking, and milestone list are in process. Proposed contributions to Canyons Disposition Initiative (CDI) characterization were investigated including gamma camera analysis.

\section{Plans for the next two months}

- Modify PTP as necessary to reflect revisions.

- Continue contact with CMST-CP, DOE focus areas, and technology vendors.

- Continue discussions and initiate plans for CDI characterization activity assimilation.

\section{FIU collaborator}

Richard Musgrove, (305) 348-6622 


\title{
Identification of DOE's Post-Closure Monitoring Needs and Requirements
}

\author{
Project Number: HCET-1998-C011
}

\section{Project objectives}

The 2006 plan sets an ambitious agenda for the U.S. Department of Energy, Office of Environmental Management's (DOE-EM) cleanup work. In the context of Accelerating Cleanup: Focus on 2006, closure refers to the completion of area- or facility-specific cleanup subtasks. The cleanup levels are determined by the planned future use of the site or facility. Many of the future land use decisions have yet to be made, though certain basic cost-based land use assumptions have been determined. Little or no EM land will be remediated to "residential use" levels; most will be remediated to "industrial use" levels with access restrictions, while some areas will be closed off through containment.

Most of the industrial use and closed-off lands will require monitoring. In the restricted and waste storage areas, the waste levels, condition, and containment will need to be monitored. In the nearby areas, groundwater and soils will need to be monitored per monitoring requirements imposed by regulators and stakeholders. Regulators will not approve closure plans without the specification of clearly defined monitoring methods using approved technologies. Therefore, inadequate planning for monitoring and the lack of appropriate monitoring technologies often prevent closure.

The current and evolving post-closure monitoring requirements at DOE-EM sites must be determined, documented, and tracked to provide the Characterization, Monitoring, and Sensor Technology Crosscutting Program (CMST-CP) with information to guide its post-closure technology development and deployment efforts. As part of this subtask, Florida International University's Hemispheric Center for Environmental Technology (FIU-HCET) will

- Determine and track post-closure monitoring needs at the Hanford, Savannah River, and Fernald sites (FY98) and the Oak Ridge (OR) and Rocky Flats (RF) sites (FY99).

\section{Major milestones}

\begin{tabular}{|c|l|l|l|}
\hline Milestone No. & \multicolumn{1}{|c|}{ Description } & \multicolumn{1}{c|}{ Completion Criteria } & \multicolumn{1}{c|}{ Status } \\
\hline C011-M1 & $\begin{array}{l}\text { Identify key post-closure } \\
\text { monitoring needs and } \\
\text { commitments at Oak Ridge. }\end{array}$ & $\begin{array}{l}\text { A report, to be included as part of the final report, } \\
\text { of the post-closure monitoring needs and } \\
\text { commitments for Oak Ridge. }\end{array}$ & $\begin{array}{l}\text { On schedule to } \\
\text { be completed } \\
\text { by } 4 / 30 / 99\end{array}$ \\
\hline C011-M2 & $\begin{array}{l}\text { Identify key post-closure } \\
\text { monitoring needs and } \\
\text { commitments at Rocky } \\
\text { Flats. }\end{array}$ & $\begin{array}{l}\text { A report, to be included as part of the final report, } \\
\text { of the post-closure monitoring needs and } \\
\text { commitments for Rocky Flats. }\end{array}$ & $\begin{array}{l}\text { On schedule to } \\
\text { be completed } \\
\text { by } 6 / 30 / 99\end{array}$ \\
\hline C011-M3 & $\begin{array}{l}\text { Identify the most common } \\
\text { post-closure monitoring } \\
\text { needs within EM }\end{array}$ & $\begin{array}{l}\text { A report, to be included as part of the final report, } \\
\text { of the most pressing post-closure needs based on } \\
\text { the five sites reviewed in FY98 and FY99. }\end{array}$ & $\begin{array}{l}\text { Scheduled to } \\
\text { begin } 6 / 30 / 99\end{array}$ \\
\hline C011-M4 & $\begin{array}{l}\text { Write the final report for the } \\
\text { project }\end{array}$ & $\begin{array}{l}\text { Report describing the post-closure needs for Oak } \\
\text { Ridge and Rocky Flats, summarizes the post- } \\
\text { closure needs for all five sites reviewed in FY98 } \\
\text { and FY99, and the most pressing post-closure } \\
\text { needs with EM }\end{array}$ & $\begin{array}{l}\text { To be } \\
\text { completed by } \\
10 / 31 / 99\end{array}$ \\
\hline
\end{tabular}




\section{Accomplishments and technical progress}

- Continued reviewing information for Oak Ridge concerning post-closure requirements and needs. Documents obtained and reviewed include post-closure plans and permits, postremediation monitoring program plans, removal action reports, fact sheets, and others. The previously planned site visit to review documents is no longer needed.

- Continued the review of Rocky Flats post-closure requirements and needs.

- Continued the identification of technology, either available or being developed, that is capable of meeting post-closure needs of $\mathrm{DOE}$ sites.

- Initiated contact with Site Technology Coordinating Group (STCG) representatives at Hanford and Savannah River for the purpose of obtaining their comments on the post-closure needs and requirements report for the respective site that was completed in FY98.

\section{Assessment of Current Status}

This project is proceeding and no scheduling deadlines have been missed. Currently, no impediments are known that could delay the on-schedule completion of milestones 1 and 2.

\section{Plans for the next two months}

- Conclude reviewing relevant documents (closure plans, post-closure plans, and Records of Decisions) for Oak Ridge. Contact different site personnel concerning post-closure needs and requirements.

- Continue reviewing information concerning the post-closure needs and requirements for the Rocky Flats site. The Technical Library and site personnel will be contacted.

- Continue the process of contacting STCG representatives at Hanford and Savannah River for purpose of obtaining their comments on the post-closure needs and requirements report for the respective site that was completed in FY98.

- Complete and release the year-end report for FY98. This report includes the post-closure needs and requirements for the Fernald, Savannah River, and Hanford sites, as well as the review of post-closure monitoring programs for Western Europe and Japan (to determine if they have any post-closure monitoring experience or innovative technology that could benefit DOE).

\section{FIU collaborator}

Hans Weger, (305) 348-6620 
MONTHLY PROGRESS REPORT

FIU Principal Investigator

FIU International Coordinator

Focus Area Technical Lead

Program Officers
M.A. Ebadian

Ana Ferreira

Elizabeth O'Malley

John Wengle

Karl-Heinz Frohne

http://www.hcet.fiu.edu 


\title{
Opportunities to Market U.S. Technologies Throughout the Western Hemisphere
}

\author{
Project Number: HCET 1996-1001
}

\section{Project objectives}

Because of its size, sophistication, and geographic proximity, the U.S. environmental industry has the potential to become a major player in the environmental markets in Latin America and the Caribbean. Building on the alliances previously established by Florida International University (FIU) with organizations in Latin America and the Caribbean, the Hemispheric Center for Environmental Technology (FIU-HCET) will work with U.S. governmental agencies and industry to develop, adapt, and market/transfer their technologies throughout the Western Hemisphere. FIU-HCET will aid government leaders of the Americas in the promotion of the use of efficient and non-polluting technologies.

FIU-HCET manages an aggressive international program for applied research, development, demonstration, testing, and evaluation. This program to identify opportunities to market U.S. technologies throughout the Western Hemisphere has been successful. It has made a number of cooperative agreements that seek to identify technologies to aid in the cleanup of DOE nuclear component manufacturing sites and, at the same time, identify technologies for international usage to work faster, safer, and cheaper than current available technologies.

This project involves an open-ended, continuous process of information gathering with respect to Latin American and Caribbean environmental issues. This entails the development of contacts with individuals and institutions conducting research and work on issues of sustainability and environmental technology in the Americas. As part of this phase, a database containing information on firms, nongovernmental organizations (NGOs), governmental institutions, and other participants in Latin America's environmental sector is being developed.

\section{Open-Ended Milestones}

\begin{tabular}{|c|l|l|l|}
\hline Milestone No. & \multicolumn{1}{|c|}{ Milestone Description } & Completion Criteria & \multicolumn{1}{c|}{ Status } \\
\hline I001-M1 & $\begin{array}{l}\text { Database: Formulate a } \\
\text { database of U.S. business } \\
\text { contacts working in the field } \\
\text { of environmental } \\
\text { technologies }\end{array}$ & $\begin{array}{l}\text { This milestone is } \\
\text { ongoing. }\end{array}$ & $\begin{array}{l}\text { Survey has been sent out to a } \\
\text { number of U.S. environmental } \\
\text { technology companies, asking } \\
\text { them to describe their } \\
\text { capabilities and market } \\
\text { experience. }\end{array}$ \\
\hline I001-M2 & $\begin{array}{l}\text { Internet Tool: Maintain the } \\
\text { Internet tool referred to as the } \\
\text { 'Virtual Secretariat' for the } \\
\text { participants of the Western } \\
\text { Hemisphere Energy } \\
\text { Symposium }\end{array}$ & $\begin{array}{l}\text { The Internet tool is to be } \\
\text { showcased at next } \\
\text { Hemispheric Energy } \\
\text { Steering Committee } \\
\text { meeting to be held } \\
\text { February 10-12 in Lima, } \\
\text { Peru. }\end{array}$ & $\begin{array}{l}\text { Working in collaboration with } \\
\text { Information Systems Managers } \\
\text { to implement final edits to } \\
\text { Internet tool. Survey was sent } \\
\text { out to Steering Committee } \\
\text { members to find out their } \\
\text { Internet capabilities to date. Due } \\
\text { date: 2/10/99 }\end{array}$ \\
\hline
\end{tabular}




\begin{tabular}{|c|l|l|l|}
\hline Milestone No. & Milestone Description & \multicolumn{1}{|c|}{ Completion Criteria } & \multicolumn{1}{c|}{ Status } \\
\hline I001-M3 & $\begin{array}{l}\text { Participate at the next } \\
\text { Hemispheric Energy Steering } \\
\text { Committee meeting in Lima, } \\
\text { Peru. }\end{array}$ & $\begin{array}{l}\text { Make a presentation of } \\
\text { the 'Virtual Secretariat' } \\
\text { and introduce FIU- } \\
\text { HCET to participants of } \\
\text { the Steering Committee, } \\
\text { announce necessity of } \\
\text { identifying future } \\
\text { funding for the Internet } \\
\text { tool and officially } \\
\text { request membership on } \\
\text { the Climate Change } \\
\text { Working Group headed } \\
\text { by Canada. }\end{array}$ & $\begin{array}{l}\text { Spoke with DOE officials on the } \\
\text { importance of identifying } \\
\text { funding. A meeting was } \\
\text { members (DOE, OLADE and } \\
\text { the Venezuelan government) to } \\
\text { discuss funding and Working } \\
\text { Group memberships. Due date: } \\
\text { 5/99. }\end{array}$ \\
& & $\begin{array}{l}\text { Identify a minimum of } \\
\text { 20 companies for } \\
\text { mission. Enterprise FL } \\
\text { has suggested that FIU- } \\
\text { HCET participate. }\end{array}$ & $\begin{array}{l}\text { Went through database, } \\
\text { identified 29 companies. Have e- } \\
\text { mailed letter to companies; } \\
\text { responses will be forwarded to } \\
\text { Enterprise FL. Due date: } 4 / 99 .\end{array}$ \\
\hline I001-M4 & $\begin{array}{l}\text { Enterprise Florida: Identify } \\
\text { U.S. companies who would } \\
\text { be interested in participating } \\
\text { in the next Export Marketing } \\
\text { Mission to Argentina, April } \\
\text { 10-16, 1999. }\end{array}$ & \\
\hline
\end{tabular}

\section{Significant events}

- Canada and Venezuela Steering Committee representatives have been assigned passwords so that they will be able to use the Member area of the Internet tool.

- DOE video entitled Seminar on New Technologies for the Environmental Sector was reviewed.

- Pictures were identified from Hemispheric Energy Initiative Working Group 2: Clean Energy Options for posting on the web site.

\section{Accomplishments and technical progress}

- As instructed by the Office of Policy, DOE, two surveys were successfully sent out to representatives of the Hemispheric Energy Steering Committee. The first survey was to determine member Internet capabilitites; the second survey asked for current events associated with the energy agenda to be sent for implementation on the web page.

- FIU-HCET personnel have been making progress on the Internet tool known as the Virtual Secretariat. Translation of the Spanish web site is on schedule, and the content on the site has been improved visually as requested by DOE representatives.

\section{Plans for the next two months}

- FIU-HCET personnel will continue to assist the DOE-EM international program manager in activities with the CNEA of Argentina. 
- FIU-HCET personnel will continue to send bi-weekly e-mails to DOE-EM on the Center's Latin American initiatives on behalf of DOE.

- FIU-HCET personnel will continue to work on the Virtual Secretariat in preparation of the Peru meeting.

\section{FIU-HCET collaborator}

Aña M. Ferreira, (305) 348-1818 